\title{
RESTRICTION ESTIMATES, SHARP SPECTRAL MULTIPLIERS AND ENDPOINT ESTIMATES FOR BOCHNER-RIESZ MEANS
}

\author{
PENG CHEN, EL MAATI OUHABAZ, ADAM SIKORA, AND LIXIN YAN
}

\begin{abstract}
AвSTRACT. We consider abstract non-negative self-adjoint operators on $L^{2}(X)$ which satisfy the finite speed propagation property for the corresponding wave equation. For such operators we introduce a restriction type condition which in the case of the standard Laplace operator is equivalent to $(p, 2)$ restriction estimate of Stein and Tomas. Next we show that in the considered abstract setting our restriction type condition implies sharp spectral multipliers and endpoint estimates for the BochnerRiesz summability. We also observe that this restriction estimate holds for operators satisfying dispersive or Strichartz estimates. We obtain new spectral multiplier results for several second order differential operators and recover some known results. Our examples include Schrödinger operators with inverse square potentials on $\mathbb{R}^{n}$, the harmonic oscillator, elliptic operators on compact manifolds and Schrödinger operators on asymptotically conic manifolds.
\end{abstract}

\section{CONTENTS}

1. Introduction 2

Part 1. Restriction estimates imply sharp spectral multipliers 7

2. Restriction type condition $\quad 7$

3. Sharp spectral multipliers - compactly supported functions 11

4. Sharp spectral multipliers - singular integral case 19

5. Endpoint estimates for Bochner-Riesz means 27

Part 2. Dispersive and restriction estimates 34

6. Dispersive and Strichartz estimates $\quad 34$

7. From dispersive and Strichartz estimates to sharp multipliers 35

Part 3. Applications 39

8. Standard Laplace operator and compact manifolds 39

9. Asymptotically conic manifolds 39

10. Schrödinger operators with rough potentials 40

11. Operators $\Delta_{n}+\frac{c}{r^{2}}$ acting on $L^{2}\left((0, \infty), r^{n-1} d r\right) \quad 43$

12. Examples of $(1,2)$-restriction type conditions 46

$\begin{array}{ll}\text { References } & 47\end{array}$

Date: November 1, 2018.

2000 Mathematics Subject Classification. 42B15, 42B20, 47F05.

Key words and phrases. Bochner-Riesz means, spectral multipliers, non-negative self-adjoint operators, finite speed propagation property, restriction type condition, dispersive and Strichartz estimates. 


\section{INTRODUCTION}

A celebrated theorem of Hörmander [35] states that for a given bounded function $F:[0, \infty) \rightarrow$ $\mathbb{C}$, the operator $F(-\Delta)$, initially defined by Fourier analysis on $L^{2}\left(\mathbb{R}^{n}\right)$, extends to a bounded operator on $L^{p}\left(\mathbb{R}^{n}\right)$ for all $p \in(1, \infty)$ provided the function satisfies

$$
\sup _{t>0}\|\eta(\cdot) F(t \cdot)\|_{W^{s, 2}}<\infty
$$

for some $s>\frac{n}{2}$. Here $\eta \in C_{c}^{\infty}(0, \infty)$ is a non-trivial auxiliary function. This result is a sharp version of the well known Mikhlin's Fourier multiplier theorem [46]. These results have led to a fruitful research activity on spectral multipliers and new perspectives in harmonic analysis. The Hörmander-Mikhlin theorem has been extended by several authors to other operators than the Laplacian and settings that go beyond the Euclidean case. The bibliography is so broad that it is impossible to provide complete list here. We refer the reader to $[2,12,14,15,16,17,21,23,24$, $25,26,27,28,30,32,34,44,47,48,50,53,54,56,58,59,61,63,64]$ and the references therein.

Suppose that $X$ is a measure space and that $L$ is a non-negative self-adjoint operator on $L^{2}(X)$. Such an operator admits a spectral resolution $E_{L}(\lambda)$ and for any bounded Borel function $F:[0, \infty) \rightarrow$ $\mathbb{C}$, one can define the operator $F(L)$

$$
F(L)=\int_{0}^{\infty} F(\lambda) \mathrm{d} E_{L}(\lambda)
$$

By the spectral theorem, $F(L)$ is well defined and bounded on $L^{2}(X)$. Spectral multiplier theorems give sufficient conditions on $F$ under which the operator $F(L)$ extends to a bounded operator on $L^{p}(X)$ for some range of $p$.

Most of the references mentioned before deal with the case of sub-Laplacians on some Lie groups. The papers $[23,24]$ deal with a rather general situation where $(X, d, \mu)$ is metric measure space of homogeneous type (or even a domain of such space). One of the results there is a spectral multiplier theorem under the sole assumption that the heat kernel of the operator has a Gaussian upper bound. The condition there is however stronger than (1.1) in the sense that the norm in $W^{s, 2}$ is replaced by the norm of $W^{s, \infty}$ where $s$ is any constant larger than half of homogeneous dimension. Under Plancherel type estimates, one obtains sharp results with condition (1.1). Condition (1.1) with norm $W^{s, 2}$ is better than the corresponding one with norm $W^{s, \infty}$. This can be seen from Bochner-Riesz summability which we discuss now.

The theory of spectral multipliers is related to and motivated by the study of convergence of Bochner-Riesz means of self-adjoint operators. Given a non-negative self-adjoint operator $L$ and set

$$
S_{R}^{\delta}(\lambda)=\left\{\begin{array}{cc}
\left(1-\frac{\lambda}{R^{2}}\right)^{\delta} & \text { for } \lambda \leq R^{2} \\
0 & \text { for } \lambda>R^{2} .
\end{array}\right.
$$

We then define the operator $S_{R}^{\delta}(L)$ using (1.2). We call $S_{R}^{\delta}(L)$ the Riesz or the Bochner-Riesz means of order $\delta$. The basic question in the theory of Bochner-Riesz means is to establish the critical exponent for the uniform continuity with respect to $R$ and convergence of the Riesz means on $L^{p}$ spaces for various $p$ with $1 \leq p \leq \infty$.

For $\delta=0$, this is the spectral projector $E_{\sqrt{L}}[0, R]$, while for $\delta>0, S_{R}^{\delta}(L)$ can be seen as a smoothed version of this spectral projector. Bochner-Riesz summability describes the range of $\delta$ for which the above operators are bounded on $L^{p}$, uniformly in $R$. If one proves a spectral multiplier result which states that $F(L)$ is bounded on $L^{p}(X)$ for all $p \in(1, \infty)$ whenever $F$ satisfies (1.1), then the Bochner-Riesz mean $S_{1}^{\delta}(L)$ is bounded on all $L^{p}$ spaces provided $\delta>\frac{n-1}{2}$. In 
the case where $L$ is the Euclidean Laplacian and $\delta>\frac{n-1}{2}$, the kernel of $S_{R}^{\delta}(L)$ is $L^{1}$ and hence Bochner-Riesz means are bounded on $L^{p}$ for $1 \leq p \leq \infty$. In this setting more is known. Indeed for $\delta>\max \left\{n\left|\frac{1}{2}-\frac{1}{p}\right|-\frac{1}{2}, 0\right\}$, it was known for a long time that as a consequence of restriction estimates for the Fourier transform, Bochner-Riesz summability holds on $L^{p}$ for all $p \leq \frac{2 n+2}{n+3}$, and by duality for $p \geq \frac{2 n+2}{n-1}$. See Stein [61], p. 420. This was extended by Lee [40] to the case $p<\frac{2 n+4}{n+4}$ (or $p>\frac{2 n+4}{n}$ ) and recent improvements are proved by Bourgain and Guth [9]. The question whether Bochner-Riesz summability holds on $L^{p}\left(\mathbb{R}^{n}\right)$ for all $p$ and all $\delta>\max \left\{n\left|\frac{1}{p}-\frac{1}{2}\right|-\frac{1}{2}, 0\right\}$ is a longstanding open problem (except for $n=2$, see Carleson and Sjölin [12] and Hörmander [33]). For all this, see Stein [61], p. 420 and the review paper of Tao [62]. The later contains many other information and relation of the Bochner-Riesz problem to other open problems in harmonic analysis.

If $L$ is a second order elliptic operator on a compact Riemannian manifold $M$ with dimension $n$, then the Bochner-Riesz means $\sum_{\lambda_{j} \leq R}\left(1-\lambda_{j} / R\right)^{\delta}<\cdot, e_{j}>e_{j}$ are uniformly bounded on $L^{p}(M)$ provided $p \leq \frac{2 n+2}{n+3}$ or by duality for $p \geq \frac{2 n+2}{n-1}$ for $\delta>\max \left\{n\left|\frac{1}{2}-\frac{1}{p}\right|-\frac{1}{2}, 0\right\}$. Here $\lambda_{0} \leq \lambda_{j} \leq \lambda_{j+1} \leq \ldots$ and $e_{j}$ are the corresponding eigenvalues and normalized $L^{2}$ eigenvectors, respectively. See Sogge [56].

The theory of Fourier multipliers and Bochner-Riesz analysis in the setting of the standard Laplace operator on $\mathbb{R}^{n}$ is related to the so-called sphere restriction problem for the Fourier transform: find the pairs $(p, q)$ for which $R_{\lambda} \in \mathcal{L}\left(L^{p}\left(\mathbb{R}^{n}\right), L^{q}\left(\mathbb{S}^{n-1}\right)\right)$ where $R_{\lambda}$ is defined by

$$
R_{\lambda} f(\omega)=\hat{f}(\lambda \omega), \omega \in \mathbb{S}^{n-1}, \lambda>0 .
$$

See for example $[25,27,59,61,62]$. For $q=2$ the full description of possible range of $p$ is due to Stein and Tomas. The theorem of Tomas [65], extended by Stein to the endpoint, states that $(p, 2)$ restriction estimates hold if and only if $1 \leq p \leq 2(n+1) /(n+3)$. The case $q \neq 2$ is not relevant to our discussion so we refer the interested reader to Tao [62] on the subject.

Note that on $\mathbb{R}^{n}$, the Schwartz kernel of the spectral measure $d E_{\sqrt{-\Delta}}(\lambda)$ of $\sqrt{-\Delta}$ is given by

$$
d E_{\sqrt{-\Delta}}\left(\lambda ; z, z^{\prime}\right)=\frac{\lambda^{n-1}}{(2 \pi)^{n}} \int_{S^{n-1}} e^{i\left(z-z^{\prime}\right) \cdot \lambda \omega} d \omega, \quad z, z^{\prime} \in \mathbb{R}^{n},
$$

therefore $d E_{\sqrt{-\Delta}}(\lambda)=\frac{\lambda^{n-1}}{(2 \pi)^{n}} R_{\lambda}^{*} R_{\lambda}$ and the restriction theorem for $q=2$ is equivalent to

$$
\left\|d E_{\sqrt{-\Delta}}(\lambda)\right\|_{p \rightarrow p^{\prime}} \leq C \lambda^{n\left(1 / p-1 / p^{\prime}\right)-1}
$$

for all $p \in\left[1, \frac{2 n+2}{n+3}\right]$. In the sequel, we refer to $(1.4)$ as $(p, 2)$ restriction estimate of Stein-Tomas.

In this paper we follow the line of research described above. We deal with the problem of sharp spectral multipliers and Bochner-Riesz summability for other operators than the Euclidean Laplacian and elliptic operators on compact manifolds. Our aim is to build a theory which applies in a rather general setting of self-adjoint operators on spaces of homogeneous type (i.e., metric measure spaces which satisfy the volume doubling property). Our approach allows us to prove sharp multiplier results and Bochner-Riesz summability in new settings and also unifies several previously known results. In order to do so we introduce a restriction type estimate which in the case of the Laplacian on $\mathbb{R}^{n}$ turns to be equivalent to the $(p, 2)$ restriction estimate of Stein-Tomas.

Our setting will be the following. We consider a non-negative self-adjoint operator $L$ on $L^{2}(X)$ where $(X, d, \mu)$ is a metric measure space which satisfies the volume doubling condition

$$
V(x, \lambda r) \leq C \lambda^{n} V(x, r) \forall x \in X, \lambda \geq 1, r>0,
$$

where $C$ and $n$ are positive constants and $V(x, r)$ denotes the volume of the open ball $B(x, r)$ of centre $x$ and radius $r$. We assume that $L$ satisfies the finite speed propagation property for 
the corresponding wave equation. We introduce the condition that for any $R>0$ and all Borel functions $F$ supported in $[0, R]$,

$\left(\mathrm{ST}_{\mathrm{p}, \mathrm{s}}^{\mathrm{q}}\right)$

$$
\left\|F(\sqrt{L}) P_{B(x, r)}\right\|_{p \rightarrow s} \leq C V(x, r)^{\frac{1}{s}-\frac{1}{p}}(R r)^{n\left(\frac{1}{p}-\frac{1}{s}\right)}\|F(R \cdot)\|_{q}
$$

for all $x \in X$ and all $r \geq 1 / R$.

We will see that if the volume is polynomial, i.e. $V(x, r) \sim r^{n}$, then $\left(\operatorname{ST}_{\mathrm{p}, 2}^{2}\right)$ is equivalent to $(p, 2)$ restriction estimate of Stein-Tomas. For this reason, we call $\left(\mathrm{ST}_{\mathrm{p}, \mathrm{s}}^{\mathrm{q}}\right)$ a Stein-Tomas restriction type condition. One of our main results on sharp spectral multipliers can be stated as follows.

Theorem A. Assume that $X$ satisfies the volume doubling condition. Suppose that L is a nonnegative self-adjoint operator which satisfies the finite speed propagation property and condition $\left(\mathrm{ST}_{\mathrm{p}, \mathrm{s}}^{\mathrm{q}}\right.$ ) for some $p, s, q$ such that $1 \leq p<s \leq \infty$ and $1 \leq q \leq \infty$.

(i) Compactly supported multipliers: Let $F$ be an even function such that $\operatorname{supp} F \subseteq[-1,1]$ and $F \in W^{\beta, q}(\mathbb{R})$ for some $\beta>n(1 / p-1 / s)$. Then $F(\sqrt{L})$ is bounded on $L^{p}(X)$, and

$$
\sup _{t>0}\|F(t \sqrt{L})\|_{p \rightarrow p} \leq C\|F\|_{W^{\beta, q}} .
$$

(ii) General multipliers: Suppose $s=2$ and $F$ is an even bounded Borel function such that $\sup _{t>0}\|\eta(\cdot) F(t \cdot)\|_{W^{\beta, q}}<\infty$ for some $\beta>\max \{n(1 / p-1 / 2), 1 / q\}$ and some non-trivial function $\eta \in C_{c}^{\infty}(0, \infty)$. Then $F(\sqrt{L})$ is bounded on $L^{r}(X)$ for all $p<r<p^{\prime}$. In addition,

$$
\|F(\sqrt{L})\|_{r \rightarrow r} \leq C_{\beta}\left(\sup _{t>0}\|\eta(\cdot) F(t \cdot)\|_{W^{\beta, q}}+|F(0)|\right) .
$$

Assertion (i) of the theorem is inspired by Guillarmou, Hassell and Sikora [28] where a related result is proved under the assumption that the volume is polynomial. Assertion (ii) is in the spirit of Hörmander's multiplier theorem for the Euclidean Laplacian. Here, if $p>1$, the order of differentiability required on $F$ is smaller since we do not search for boundedness of $F(\sqrt{L})$ on $L^{r}$ for all $r \in(1, \infty)$.

The proof of assertion (i) makes heavy use of the finite speed propagation property. This property together with the classical dyadic decomposition of $F$ allow to reduce the problem of boundedness of $F(\sqrt{L})$ on $L^{p}$ to boundedness of certain compactly supported operators. The SteinTomas restriction type condition will be used to obtain an $L^{p}-L^{s}$ estimate of these operators from which we recover the boundedness of $F(\sqrt{L})$ on $L^{p}$.

Assertion (ii) appeals as expected to singular integral theory. We shall also make use of the estimate from assertion (i) since $F(\sqrt{L})$ can be written as the sum $\sum F_{j}(\sqrt{L})$ with compactly supported functions $F_{j}$. However the operators $F_{j}(\sqrt{L})$ do not act independently of each other and hence $L^{p}$ estimate for $F(\sqrt{L})$ does not hold in a trivial way from the corresponding estimates for $F_{j}(\sqrt{L})$. As explained by Littman, McCarthy and Rivière [42], we may have $F_{j}(\sqrt{-\Delta})$ to be uniformly bounded on $L^{1}\left(\mathbb{R}^{n}\right)$ but $F(\sqrt{-\Delta})$ fails to be a multiplier of any $L^{p}$ other than $L^{2}$. This problem of recovering $L^{p}$ bounds for $F(\sqrt{-\Delta})$ from those for $F_{j}(\sqrt{-\Delta})$ is discussed by Carbery, Seeger and Sogge in $[11,52,53]$. We shall follow closely Carbery [11] and adapt some ideas there to our abstract setting.

Our restriction type estimate does not hold when the set of point spectrum is not empty. In particular, it does not hold for elliptic operators on compact manifolds or for the harmonic oscillator. In order to treat these situations as well we modify the restriction estimate as follows: for a fixed natural number $\kappa$ and for all $N \in \mathbb{N}$ and all even Borel functions $F$ such that $\operatorname{supp} F \subseteq[-N, N]$,

$\left(\mathrm{SC}_{\mathrm{p}, \mathrm{s}}^{\mathrm{q}, \kappa}\right) \quad\left\|F(\sqrt{L}) P_{B(x, r)}\right\|_{p \rightarrow s} \leq C V(x, r)^{\frac{1}{s}-\frac{1}{p}}(N r)^{n\left(\frac{1}{p}-\frac{1}{s}\right)}\|F(N \cdot)\|_{N^{\kappa}, q}$, 
for all $x \in X$ and all $r \geq 1 / N$ where

$$
\|F\|_{N, q}=\left(\frac{1}{2 N} \sum_{\ell=1-N}^{N} \sup _{\lambda \in\left[\frac{\ell-1}{N}, \frac{\ell}{N}\right)}|F(\lambda)|^{q}\right)^{1 / q}
$$

for $F$ supported in $[-1,1]$. For $q=\infty$, we put $\|F\|_{N, \infty}=\|F\|_{\infty}$. The norm $\|F\|_{N, q}$ was used by Cowling and Sikora [21] and Duong, Ouhabaz and Sikora [23] in the setting of spectral multipliers.

In some situations, $\left(\mathrm{SC}_{\mathrm{p}, 2}^{2,1}\right)$ is equivalent to the following condition introduced by Sogge (see $[56,57,58])$

$$
\left\|E_{\sqrt{L}}[\lambda, \lambda+1)\right\|_{p \rightarrow p^{\prime}} \leq C(1+\lambda)^{n\left(\frac{1}{p}-\frac{1}{p^{\prime}}\right)-1} .
$$

We call $\left(\mathrm{SC}_{\mathrm{p}, \mathrm{s}}^{\mathrm{q}, \kappa}\right)$ Sogge's spectral cluster condition. In this context we shall prove the following result (see Theorems 3.6 and 4.2 for precise statements).

Theorem B. Suppose that $X$ has finite measure and satisfies the volume doubling condition. Let $L$ be a non-negative self-adjoint operator which satisfies the finite speed propagation property and Sogge's spectral cluster condition ( $\mathrm{SC}_{\mathrm{p}, \mathrm{s}}^{\mathrm{q}, 1}$ ) for some $p, s, q$ such that $1 \leq p<s \leq \infty$ and $1 \leq q \leq \infty$. Then both assertions of Theorem A hold provided $\beta>\max \{n(1 / p-1 / s), 1 / q\}$.

The same conclusion holds in the case where $\mu(X)=\infty$ provided $\left(\mathrm{SC}_{\mathrm{p}, \mathrm{s}}^{\mathrm{q}, \kappa}\right)$ and an a priori estimate for $\|F(\sqrt{L})\|_{p \rightarrow p}$ are satisfied.

As for Theorem A, an appropriate decomposition of $F(\sqrt{L})$ as the sum of operators with compact supports is the backbone of our arguments in proving boundedness of $F(\sqrt{L})$ on $L^{p}$ for compactly supported $F$. Passing from compactly supported multipliers to the general case will be done in the same way as for Theorem A. The proof of this part does not make explicit use of $\left(\mathrm{ST}_{\mathrm{p}, 2}^{\mathrm{q}}\right)$ or $\left(\mathrm{SC}_{\mathrm{p}, 2}^{\mathrm{q}, K}\right)$ but the rather weaker condition

$$
\left\|(I+t \sqrt{L})^{-N} P_{B(x, r)}\right\|_{p \rightarrow 2} \leq C V(x, r)^{\frac{1}{2}-\frac{1}{p}}\left(\frac{r}{t}\right)^{n\left(\frac{1}{p}-\frac{1}{2}\right)}, x \in X, r \geq t>0 .
$$

Starting now from Theorem A or Theorem B with $s=2$ and choosing the function $F=S_{R}^{\delta}$ yields Bochner-Riesz summability on $L^{p}(X)$ for $\delta>\delta_{q}(p)$ where

$$
\delta_{q}(p)=\max \left\{0, n\left|\frac{1}{p}-\frac{1}{2}\right|-\frac{1}{q}\right\} .
$$

Now we address the question of endpoint estimates, i.e., estimate for $S_{R}^{\delta_{q}(p)}(L)$. It turns out that our Stein-Tomas restriction type condition or Sogge's cluster condition imply that Bochner-Riesz means are weak-type $(p, p)$ operators for $\delta=\delta_{q}(p)$. More precisely we obtain

Theorem C. Assume that $X$ satisfies the doubling condition and operator L satisfies the finite speed propagation property.

(i) If the restriction condition $\left(\mathrm{ST}_{\mathrm{p}, 2}^{\mathrm{q}}\right.$ ) holds for some $p$, $q$ satisfying $1 \leq p<2$ and $1 \leq q \leq \infty$ then $S_{R}^{\delta_{q}(p)}(L)$ is of weak-type ( $\left.p, p\right)$ uniformly in $R$.

(ii) The same conclusion as in (i) holds if $\mu(X)<\infty$ and $\left(\mathrm{SC}_{\mathrm{p}, 2}^{\mathrm{q}, 1}\right)$ is satisfied for some $p, q$ satisfying $1 \leq p<2$ and $1 \leq q \leq \infty$.

In the Euclidean case, it is known that $S_{R}^{\delta_{2}(p)}(-\Delta)$ is not bounded on $L^{p}\left(\mathbb{R}^{n}\right)$ for $p \neq 2$. This was observed by Christ and Sogge [17] who also proved weak-type $(1,1)$ for $S_{R}^{\delta_{2}(1)}(-\Delta)$. Weak-type $(p, p)$ estimates of $S_{R}^{\delta_{2}(p)}(-\Delta)$ are proved by Christ $[14,15]$ when $p<\frac{2 n+2}{n+3}$. The corresponding 
result on compact manifolds is proved by Seeger [51]. The endpoint estimates for $p=\frac{2 n+2}{n+3}$ are proved by Tao [63] both for $\mathbb{R}^{n}$ and compact manifolds.

Our approach for endpoint estimates is inspired by Christ and Tao [14, 15, 63]. It is based on $L^{2}$ Calderón-Zygmund techniques (as used in Fefferman [25]), a spacial decomposition of the Bochner-Riesz multiplier and the fact that if $F$ has its inverse Fourier transform supported on a set of width $R$, then by the finite speed propagation property the operator $F(\sqrt{L})$ is supported in a $C R$ - neighbourhood of the diagonal. It is worth to note that our proof of endpoint estimates does not require any cancellation argument. This allows us to consider applications to operators with non-smooth kernels.

The previous theorems are proved in Part 1 of this paper. In Part 2, we investigate the relation of $\left(\mathrm{ST}_{\mathrm{p}, 2}^{2}\right)$ to dispersive or Strichartz estimates for the corresponding Schrödinger equation

$$
\partial_{t} u+i L u=0, u(0)=f \in L^{2} .
$$

In the setting of Euclidean Laplacian, Strichartz's original proof for $L^{p}\left(\mathbb{R} \times \mathbb{R}^{n}\right)$ estimates of the solution $u$ of (1.5) uses restriction estimates of the Fourier transform. In some sense we want to do the converse here, we want to take advantage of known dispersive or Strichartz estimates for (1.5) to prove a Stein-Tomas restriction type condition and then obtain sharp spectral multipliers by Theorem A. We are able do this either directly from dispersive estimate for $e^{i t L}$ or from endpoint Strichartz estimate. We prove the following result.

\section{Theorem D.}

(i) Suppose that L satisfies the Strichartz estimate

$$
\int_{\mathbb{R}}\left\|e^{i t L} f\right\|_{\frac{2 n}{n-2}}^{2} d t \leq C\|f\|_{2}^{2}, f \in L^{2}
$$

for some $n>2$. Assume also that the smoothing property

$$
\|\exp (-t L)\|_{p \rightarrow \frac{2 n}{n+2}} \leq K t^{-\frac{n}{2}\left(\frac{1}{p}-\frac{n+2}{2 n}\right)},
$$

holds for all $p \in\left[1, \frac{2 n}{n+2}\right]$. Then for all $\lambda \geq 0$

$$
\left\|d E_{\sqrt{L}}(\lambda)\right\|_{p \rightarrow p^{\prime}} \leq C \lambda^{n\left(\frac{1}{p}-\frac{1}{p^{\prime}}\right)-1} .
$$

(ii) Fix $p \in\left[1, \frac{2 n}{n+2}\right]$. Suppose that $X$ satisfies the doubling condition and that there exists a positive constants $C>0$ such that $V(x, r) \leq C r^{n}$ for every $x \in X$ and $r>0$. Assume that $L$ satisfies the finite speed propagation property together with Strichartz and smoothing estimates as in (i). Then for every even compactly supported bounded function $F$ such that $\|F\|_{W^{\beta, 2}}<\infty$ for some $\beta>n\left(\frac{1}{p}-\frac{1}{2}\right)$, the operator $F(\sqrt{L})$ is bounded on $L^{p}$ and

$$
\sup _{t>0}\|F(t \sqrt{L})\|_{p \rightarrow p} \leq C\|F\|_{W^{\beta, 2}} \text {. }
$$

(iii) Suppose that the conditions of (ii) are satisfied. Then for any even bounded Borel function $F$ such that $\sup _{t>0}\|\eta(.) F(t \cdot)\|_{W^{\beta, 2}}<\infty$ for some $\beta>\max \{n(1 / p-1 / 2), 1 / 2\}$ and some nontrivial function $\eta \in C_{c}^{\infty}(0, \infty)$, the operator $F(\sqrt{L})$ is bounded on $L^{r}(X)$ for all $r \in\left(p, p^{\prime}\right)$.

The main assertion here is (i). Indeed, once (i) is proved we obtain a Stein-Tomas restriction type estimate and then appeal to Theorem A to prove assertions (ii) and (iii). We can also replace the Strichartz estimate by a dispersive estimate

$$
\left\|e^{i t L}\right\|_{1 \rightarrow \infty} \leq C|t|^{-n / 2}, \quad t \in \mathbb{R}, t \neq 0 .
$$


Note that by a result of Keel and Tao [37], endpoint Strichartz estimate follow from this dispersive estimate.

Strichartz estimates have been studied by several authors. For example, Burq, Planchon, Stalker and A. Tahvildar-Zadeh [10] proved such estimates for Schrödinger operators with inverse square potentials, i.e. $L=-\Delta+\frac{c}{|x|^{2}}$ on $\mathbb{R}^{n}$. Therefore we obtain sharp multiplier results as well as endpoint Bochner-Riesz estimates for these operators. It is worth to mention that if $-(n-2)^{2} / 4<c<0$, the semigroup $\exp (-t L)$ acts on $L^{p}\left(\mathbb{R}^{n}\right)$ only for $p \in\left(p_{c}^{\prime}, p_{c}\right)$ with $p_{c}<\infty$. In particular, the corresponding heat kernel does not enjoy any good upper bounds such as Gaussian upper bounds. Nevertheless we obtain sharp spectral multipliers for $L$ on $L^{p}$ for $p \in\left(p_{c}^{\prime}, 2 n /(n+2)\right]$. We discuss in Part 3 several other examples to which Theorems A, B, C and D apply. This includes radial Schrödinger operators with inverse square potentials, the harmonic oscillator, elliptic operators on compact manifolds, Laplacian on asymptotically conic manifolds.

While this paper was finished we learned that M. Uhl introduced recently in his PhD Thesis [67] a condition similar to our restriction type condition and proved a spectral multiplier result similar to our Theorems 4.1 and 4.2. However the order of differentiability required in Uhl's result is $\beta>n / 2$ and hence it is less sharp than our Theorems 4.1 and 4.2.

\section{Part 1. Restriction estimates imply sharp spectral multipliers}

\section{RESTRICTION TYPE CONDITION}

We start by fixing some notation and assumptions. Throughout this paper, unless we mention the contrary, $(X, d, \mu)$ is a metric measure space, that is, $\mu$ is a Borel measure with respect to the topology defined by the metric $d$. We denote by $B(x, r)=\{y \in X, d(x, y)<r\}$ the open ball with centre $x \in X$ and radius $r>0$. We often just use $B$ instead of $B(x, r)$. Given $\lambda>0$, we write $\lambda B$ for the $\lambda$-dilated ball which is the ball with the same centre as $B$ and radius $\lambda r$. We set $V(x, r)=\mu(B(x, r))$ the volume of $B(x, r)$ and we say that $(X, d, \mu)$ satisfies the doubling property (see Chapter 3, [18]) if there exists a constant $C>0$ such that

$$
V(x, 2 r) \leq C V(x, r) \quad \forall r>0, x \in X .
$$

If this is the case, there exist $C, n$ such that for all $\lambda \geq 1$ and $x \in X$

$$
V(x, \lambda r) \leq C \lambda^{n} V(x, r) .
$$

In the sequel we want to consider $n$ as small as possible. Note that in general one cannot take infimum over such exponents $n$ in (2.2). In the Euclidean space with Lebesgue measure, $n$ corresponds to the dimension of the space. Observe that if $X$ satisfies (2.1) and has finite measure then it has finite diameter (see, e.g., [3]). Therefore if $\mu(X)$ is finite, then we may assume that $X=B\left(x_{0}, 1\right)$ for some $x_{0} \in X$.

For $1 \leq p \leq+\infty$, we denote the norm of a function $f \in L^{p}(X, \mathrm{~d} \mu)$ by $\|f\|_{p}$, by $\langle.,$.$\rangle the$ scalar product of $L^{2}(X, \mathrm{~d} \mu)$, and if $T$ is a bounded linear operator from $L^{p}(X, \mathrm{~d} \mu)$ to $L^{q}(X, \mathrm{~d} \mu)$, $1 \leq p, q \leq+\infty$, we write $\|T\|_{p \rightarrow q}$ for the operator norm of $T$. Given a subset $E \subseteq X$, we denote by $\chi_{E}$ the characteristic function of $E$ and set

$$
P_{E} f(x)=\chi_{E}(x) f(x) .
$$

For a given function $F: \mathbb{R} \rightarrow \mathbb{C}$ and $R>0$, we define the function $\delta_{R} F: \mathbb{R} \rightarrow \mathbb{C}$ by putting $\delta_{R} F(x)=F(R x)$. 
2.1. Finite speed propagation for the wave equation. Set

$$
\mathcal{D}_{\rho}=\{(x, y) \in X \times X: d(x, y) \leq \rho\} .
$$

Given an operator $T$ from $L^{p}(X)$ to $L^{q}(X)$, we write

$$
\operatorname{supp} K_{T} \subseteq \mathcal{D}_{\rho}
$$

if $\left\langle T f_{1}, f_{2}\right\rangle=0$ whenever $f_{k}$ is in $C(X)$ and has support supp $f_{k} \subseteq B\left(x_{k}, \rho_{k}\right)$ when $k=1,2$, and $\rho_{1}+\rho_{2}+\rho<d\left(x_{1}, x_{2}\right)$. Note that if $T$ is an integral operator with a kernel $K_{T}$, then (2.3) coincides with the standard meaning of $\operatorname{supp} K_{T} \subseteq \mathcal{D}_{\rho}$, that is $K_{T}(x, y)=0$ for all $(x, y) \notin \mathcal{D}_{\rho}$.

Given a non-negative self-adjoint operator $L$ on $L^{2}(X)$. We say that $L$ satisfies the finite speed propagation property if

$$
\operatorname{supp} K_{\cos (t \sqrt{L})} \subseteq \mathcal{D}_{t} \quad \forall t>0 .
$$

Property (FS) holds for most of second order self-adjoint operators and is equivalent to DaviesGaffney estimates. See, for example [13], [55] and [19].

We recall the following well-known simple lemma.

Lemma 2.1. Assume that $L$ satisfies (FS) and that $F$ is an even bounded Borel function with Fourier transform $\hat{F} \in L^{1}(\mathbb{R})$ and that $\operatorname{supp} \hat{F} \subseteq[-\rho, \rho]$. Then

$$
\operatorname{supp} K_{F(\sqrt{L})} \subseteq \mathcal{D}_{\rho} .
$$

Proof. If $F$ is an even function, then by the Fourier inversion formula,

$$
F(\sqrt{L})=\frac{1}{2 \pi} \int_{-\infty}^{+\infty} \hat{F}(t) \cos (t \sqrt{L}) d t .
$$

But supp $\hat{F} \subseteq[-\rho, \rho]$, and the lemma follows then from (FS).

2.2. The Stein-Tomas restriction type condition. Assume that $(X, d, \mu)$ satisfies the doubling condition, that is (2.2). Consider a non-negative self-adjoint operator $L$ and numbers $p, s$ and $q$ such that $1 \leq p<s \leq \infty$ and $1 \leq q \leq \infty$. We say that $L$ satisfies the Stein-Tomas restriction type condition if: for any $R>0$ and all Borel functions $F$ such that $\operatorname{supp} F \subset[0, R]$,

$$
\left\|F(\sqrt{L}) P_{B(x, r)}\right\|_{p \rightarrow s} \leq C V(x, r)^{\frac{1}{s}-\frac{1}{p}}(R r)^{n\left(\frac{1}{p}-\frac{1}{s}\right)}\left\|\delta_{R} F\right\|_{q}
$$

for all $x \in X$ and all $r \geq 1 / R$.

Remark 2.2. Note that if condition $\left(\mathrm{ST}_{\mathrm{p}, \mathrm{s}}^{\mathrm{q}}\right)$ holds for some $q \in[1, \infty)$, then $\left(\mathrm{ST}_{\mathrm{p}, \mathrm{s}}^{\tilde{\mathrm{q}}}\right)$ holds for all $\tilde{q} \geq q$ including the case $\tilde{q}=\infty$.

Proposition 2.3. Suppose that $(X, d, \mu)$ satisfies property (2.2). Let $1 \leq p<2$ and $N>n(1 / p-$ $1 / 2)$. Then $\left(\mathrm{ST}_{\mathrm{p}, 2}^{\infty}\right)$ is equivalent to each of the following conditions:

(a) For all $x \in X$ and $r \geq t>0$ we have

$$
\left\|e^{-t^{2} L} P_{B(x, r)}\right\|_{p \rightarrow 2} \leq C V(x, r)^{\frac{1}{2}-\frac{1}{p}}\left(\frac{r}{t}\right)^{n\left(\frac{1}{p}-\frac{1}{2}\right)} .
$$

(b) For all $x \in X$ and $r \geq t>0$ we have

$$
\left\|(I+t \sqrt{L})^{-N} P_{B(x, r)}\right\|_{p \rightarrow 2} \leq C V(x, r)^{\frac{1}{2}-\frac{1}{p}}\left(\frac{r}{t}\right)^{n\left(\frac{1}{p}-\frac{1}{2}\right)} .
$$


Proof. We shall show that $\left(\mathrm{E}_{\mathrm{p}, 2}\right) \Rightarrow\left(\mathrm{ST}_{\mathrm{p}, 2}^{\infty}\right) \Rightarrow\left(\mathrm{G}_{\mathrm{p}, 2}\right) \Rightarrow\left(\mathrm{E}_{\mathrm{p}, 2}\right)$.

Suppose that $F$ is a Borel function with $\operatorname{supp} F \subset[0, R]$. Let $1 \leq p<2$ and $N>n(1 / p-1 / 2)$. It follows from $\left(\mathrm{E}_{\mathrm{p}, 2}\right)$ that for every $x \in X$ and $r \geq 1 / R$,

$$
\begin{aligned}
\left\|F(\sqrt{L}) P_{B(x, r)}\right\|_{p \rightarrow 2} & =\left\|F(\sqrt{L})\left(I+\frac{\sqrt{L}}{R}\right)^{N}\left(I+\frac{\sqrt{L}}{R}\right)^{-N} P_{B(x, r)}\right\|_{p \rightarrow 2} \\
& \leq \sup _{\lambda}\left|F(\lambda)\left(1+\frac{\lambda}{R}\right)^{N}\right| \cdot\left\|\left(I+\frac{\sqrt{L}}{R}\right)^{-N} P_{B(x, r)}\right\|_{p \rightarrow 2} \\
& \leq C 2^{N} V(x, r)^{\frac{1}{2}-\frac{1}{p}}(R r)^{n\left(\frac{1}{p}-\frac{1}{2}\right)}\left\|\delta_{R} F\right\|_{\infty} .
\end{aligned}
$$

This gives condition $\left(\mathrm{ST}_{\mathrm{p}, 2}^{\infty}\right)$.

Next assume $\left(\mathrm{ST}_{\mathrm{p}, 2}^{\infty}\right)$. Then

$$
\begin{aligned}
\left\|e^{-t^{2} L} P_{B(x, r)}\right\|_{p \rightarrow 2} & =\left\|\int_{0}^{\infty} e^{-t^{2} \lambda} d E_{L}(\lambda) P_{B(x, r)}\right\|_{p \rightarrow 2} \\
& =\left\|\int_{0}^{\infty} t^{2} e^{-t^{2} \lambda} E_{L}[0, \lambda] P_{B(x, r)} d \lambda\right\|_{p \rightarrow 2} \\
& \leq \int_{0}^{\infty} t^{2} e^{-t^{2} \lambda}\left\|\chi_{[0, \sqrt{\lambda}]}(\sqrt{L}) P_{B(x, r)}\right\|_{p \rightarrow 2} d \lambda .
\end{aligned}
$$

Now if $0<\lambda \leq 1 / r^{2}$, then $B(x, r) \subseteq B\left(x, \lambda^{-1 / 2}\right)$. Therefore, by $\left(\mathrm{ST}_{\mathrm{p}, 2}^{\infty}\right)$

$$
\begin{aligned}
\left\|\chi_{[0, \sqrt{\lambda}]}(\sqrt{L}) P_{B(x, r)}\right\|_{p \rightarrow 2} & \leq\left\|\chi_{[0, \sqrt{\lambda}]}(\sqrt{L}) P_{B\left(x, \lambda^{-1 / 2}\right)}\right\|_{p \rightarrow 2} \\
& \leq C V\left(x, \lambda^{-1 / 2}\right)^{\frac{1}{2}-\frac{1}{p}} \leq C V(x, r)^{\frac{1}{2}-\frac{1}{p}} .
\end{aligned}
$$

If $\lambda>1 / r^{2}$, then by $\left(\mathrm{ST}_{\mathrm{p}, 2}^{\infty}\right)$

$$
\left\|\chi_{[0, \sqrt{\lambda}]}(\sqrt{L}) P_{B(x, r)}\right\|_{p \rightarrow 2} \leq C V(x, r)^{\frac{1}{2}-\frac{1}{p}}\left(r \lambda^{1 / 2}\right)^{n\left(\frac{1}{p}-\frac{1}{2}\right)} .
$$

This proves $\left(\mathrm{G}_{\mathrm{p}, 2}\right)$.

To finish the proof assume that $\left(\mathrm{G}_{\mathrm{p}, 2}\right)$ holds. Then

$$
\begin{aligned}
\left\|(I+t \sqrt{L})^{-N} P_{B(x, r)}\right\|_{p \rightarrow 2} & \leq\left\|\left(I+t^{2} L\right)^{N / 2}(I+t \sqrt{L})^{-N}\left(I+t^{2} L\right)^{-N / 2} P_{B(x, r)}\right\|_{p \rightarrow 2} \\
& \leq \sup _{\lambda}\left|\left(1+t^{2} \lambda^{2}\right)^{N / 2}(1+t \lambda)^{-N}\right| \cdot\left\|\left(I+t^{2} L\right)^{-\frac{N}{2}} P_{B(x, r)}\right\|_{p \rightarrow 2} \\
& \leq C\left\|\left(I+t^{2} L\right)^{-N / 2} P_{B(x, r)}\right\|_{p \rightarrow 2} .
\end{aligned}
$$

Next note that for $t>0$

$$
\begin{aligned}
\left\|\left(I+t^{2} L\right)^{-N / 2} P_{B(x, r)}\right\|_{p \rightarrow 2} & =\left\|C_{N} \int_{0}^{\infty} e^{-s} s^{N / 2-1} e^{-s t^{2} L} P_{B(x, r)} d s\right\|_{p \rightarrow 2} \\
& \leq C_{N} \int_{0}^{\infty} e^{-s} s^{N / 2-1}\left\|e^{-s t^{2} L} P_{B(x, r)}\right\|_{p \rightarrow 2} d s .
\end{aligned}
$$

Hence if $s \leq r^{2} / t^{2}$, then by $\left(\mathrm{G}_{\mathrm{p}, 2}\right)$

$$
\left\|e^{-s t^{2} L} P_{B(x, r)}\right\|_{p \rightarrow 2} \leq V(x, r)^{\frac{1}{2}-\frac{1}{p}}\left(\frac{r}{t s^{1 / 2}}\right)^{n\left(\frac{1}{p}-\frac{1}{2}\right)} .
$$

If $s>r^{2} / t^{2}$, then $B(x, r) \subseteq B\left(x, t s^{1 / 2}\right)$ and $\left(\mathrm{G}_{\mathrm{p}, 2}\right)$ implies that

$$
\left\|e^{-s t^{2} L} P_{B(x, r)}\right\|_{p \rightarrow 2} \leq\left\|e^{-s t^{2} L} P_{B\left(x, t s^{1 / 2}\right)}\right\|_{p \rightarrow 2} \leq C V\left(x, t s^{1 / 2}\right)^{\frac{1}{2}-\frac{1}{p}} \leq C V(x, r)^{\frac{1}{2}-\frac{1}{p}} .
$$


Using these estimates in (2.4) yields $\left(\mathrm{E}_{\mathrm{p}, 2}\right)$ for $N>n(1 / p-1 / 2)$. This ends the proof.

It is natural to generalise condition (1.4) to abstract self-adjoint operators in the following way (see [28]). One says that $L$ satisfies $L^{p}$ to $L^{p^{\prime}}$ restriction estimates if the spectral measure $d E_{\sqrt{L}}(\lambda)$ maps $L^{p}(X)$ to $L^{p^{\prime}}(X)$ for some $p<2$, with an operator norm estimate

$$
\left\|d E_{\sqrt{L}}(\lambda)\right\|_{p \rightarrow p^{\prime}} \leq C \lambda^{n\left(\frac{1}{p}-\frac{1}{p^{\prime}}\right)-1}
$$

for all $\lambda \geq 0$, where $n$ is as in condition (2.2) and $p^{\prime}$ is conjugate of $p$, i.e., $1 / p+1 / p^{\prime}=1$.

Proposition 2.4. Fix $1 \leq p<2 n /(n+1)$. Suppose that there exists a constant $C>0$ such that $C^{-1} r^{n} \leq V(x, r) \leq C r^{n}$ for all $x \in X$ and $r>0$. Then conditions $\left(\mathrm{R}_{\mathrm{p}}\right),\left(\mathrm{ST}_{\mathrm{p}, 2}^{2}\right)$ and $\left(\mathrm{ST}_{\mathrm{p}, \mathrm{p}^{\prime}}^{1}\right)$ are equivalent.

Proof. The proof is inspired by estimates (2.12) of [28]. We first show the implication $\left(\mathrm{R}_{\mathrm{p}}\right) \Rightarrow$ $\left(\mathrm{ST}_{\mathrm{p}, \mathrm{p}^{\prime}}^{1}\right)$. Suppose that $F$ is a Borel function such that $\operatorname{supp} F \subset[0, R]$ for some $R>0$. Then by $\left(\mathrm{R}_{\mathrm{p}}\right)$

$$
\begin{aligned}
\left\|F(\sqrt{L}) P_{B(x, r)}\right\|_{p \rightarrow p^{\prime}} & \leq \int_{0}^{\infty} \mid F(\lambda)\left\|d E_{\sqrt{L}}(\lambda)\right\|_{p \rightarrow p^{\prime}} d \lambda \\
& \leq C \int_{0}^{R}|F(\lambda)| \lambda^{n\left(\frac{1}{p}-\frac{1}{p^{\prime}}\right)-1} d \lambda \\
& \leq C R^{n\left(\frac{1}{p}-\frac{1}{p^{\prime}}\right)-1} \int_{0}^{R}|F(\lambda)| d \lambda \\
& \leq C V(x, r)^{\frac{1}{p^{\prime}}-\frac{1}{p}}(r R)^{n\left(\frac{1}{p}-\frac{1}{p^{\prime}}\right)}\left\|\delta_{R} F\right\|_{1},
\end{aligned}
$$

where in the last inequality we used the assumption that $V(x, r) \leq C r^{n}$.

Next we prove that $\left(\mathrm{ST}_{\mathrm{p}, \mathrm{p}^{\prime}}^{1}\right) \Rightarrow\left(\mathrm{ST}_{\mathrm{p}, 2}^{2}\right)$. Note that $V(x, r) \sim r^{n}$ for every $x \in X$ and $r>0$. Letting $r \rightarrow \infty$ we obtain from $\left(\mathrm{ST}_{\mathrm{p}, \mathrm{p}^{\prime}}^{1}\right)$

By $T^{*} T$ argument

$$
\|F(\sqrt{L})\|_{p \rightarrow p^{\prime}} \leq C R^{n\left(\frac{1}{p}-\frac{1}{p^{\prime}}\right)}\left\|\delta_{R} F\right\|_{1} .
$$

$$
\|F(\sqrt{L})\|_{p \rightarrow 2}^{2}=\left\||F|^{2}(\sqrt{L})\right\|_{p \rightarrow p^{\prime}} \leq C R^{2 n\left(\frac{1}{p}-\frac{1}{2}\right)}\left\|\delta_{R} F\right\|_{2}^{2} .
$$

Hence

$$
\left\|F(\sqrt{L}) P_{B(x, r)}\right\|_{p \rightarrow 2} \leq\|F(\sqrt{L})\|_{p \rightarrow 2} \leq C V(x, r)^{\frac{1}{2}-\frac{1}{p}}(R r)^{n\left(\frac{1}{p}-\frac{1}{2}\right)}\left\|\delta_{R} F\right\|_{2} .
$$

This gives $\left(\mathrm{ST}_{\mathrm{p}, 2}^{2}\right)$.

Now, we prove the remaining implication $\left(\mathrm{ST}_{\mathrm{p}, 2}^{2}\right) \Rightarrow\left(\mathrm{R}_{\mathrm{p}}\right)$. By volume estimate $V(x, r) \geq C^{-1} r^{n}$ and condition $\left(\mathrm{ST}_{\mathrm{p}, 2}^{2}\right)$

$$
\left\|F(\sqrt{L}) P_{B(x, r)}\right\|_{p \rightarrow 2} \leq C R^{n\left(\frac{1}{p}-\frac{1}{2}\right)}\left\|\delta_{R} F\right\|_{2}
$$

for any $R>0$, all Borel functions $F$ such that $\operatorname{supp} F \subset[0, R]$, all $x \in X$ and $r \geq 1 / R$. Taking the limit $r \rightarrow \infty$ gives

$$
\|F(\sqrt{L})\|_{p \rightarrow 2} \leq C R^{n\left(\frac{1}{p}-\frac{1}{2}\right)}\left\|\delta_{R} F\right\|_{2} .
$$

For $F=\chi_{(\lambda-\varepsilon, \lambda+\varepsilon]}$ and $R=\lambda+\varepsilon$ in (2.6) yields

$$
\left\|\varepsilon^{-1} E_{\sqrt{L}}(\lambda-\varepsilon, \lambda+\varepsilon]\right\|_{p \rightarrow p^{\prime}}=\varepsilon^{-1}\left\|E_{\sqrt{L}}(\lambda-\varepsilon, \lambda+\varepsilon]\right\|_{p \rightarrow 2}^{2}
$$




$$
\begin{aligned}
& \leq C \varepsilon^{-1}(\lambda+\varepsilon)^{2 n\left(\frac{1}{p}-\frac{1}{2}\right)}\left\|\chi_{\left(\frac{\lambda-\varepsilon}{\lambda+\varepsilon}, 1\right]}\right\|_{2}^{2} \\
& \leq C(\lambda+\varepsilon)^{n\left(\frac{1}{p}-\frac{1}{p^{\prime}}\right)-1} .
\end{aligned}
$$

Taking $\varepsilon \rightarrow 0$ yields condition $\left(\mathrm{R}_{\mathrm{p}}\right)$ (see Proposition 1 , Chapter XI, [68]).

\section{SHARP SPECTRAL MULTIPLIERS - COMPACTLY SUPPORTED FUNCTIONS}

In this section we show that the restriction type condition which we introduce in the previous section can be used to obtain sharp spectral multiplier results in the abstract setting of self-adjoint operators acting on homogeneous spaces. We first consider the case of compactly supported functions. We assume here that $(X, d, \mu)$ is a metric measure space satisfying the doubling property and recall that $n$ is the doubling dimension from condition $(2.2)$. We use the standard notation $W^{\beta, q}(\mathbb{R})$ for the Sobolev space $\|F\|_{W^{\beta, q}}=\left\|\left(I-d^{2} / d x^{2}\right)^{\beta / 2} F\right\|_{q}$. The first result and its proof are inspired by Theorem 1.1 of [28].

Theorem 3.1. Suppose that operator L satisfies property (FS) and condition $\left(\mathrm{ST}_{\mathrm{p}, \mathrm{s}}^{\mathrm{q}}\right)$ for some $p, s, q$ such that $1 \leq p<s \leq \infty$ and $1 \leq q \leq \infty$. Next assume that $F$ is an even function such that $\operatorname{supp} F \subseteq[-1,1]$ and $F \in W^{\beta, q}(\mathbb{R})$ for some $\beta>n(1 / p-1 / s)$. Then $F(t \sqrt{L})$ is bounded on $L^{p}(X)$ for all $t>0$. In addition,

$$
\sup _{t>0}\|F(t \sqrt{L})\|_{p \rightarrow p} \leq C\|F\|_{W^{\beta, q}} .
$$

We described the proof of the Theorem 3.1 at the end of this section.

A standard application of spectral multiplier theorems is Bochner-Riesz means. Such application is also a good test to check if the considered multiplier result is sharp. Let us recall that Bochner-Riesz means of order $\delta$ for a non-negative self-adjoint operator $L$ are defined by the formula

$$
S_{R}^{\delta}(L)=\left(I-\frac{L}{R^{2}}\right)_{+}^{\delta}, \quad R>0 .
$$

The case $\delta=0$ corresponds to the spectral projector $E_{\sqrt{L}}[0, R]$. For $\delta>0$ we think of (3.2) as a smoothed version of this spectral projector; the larger $\delta$, the more smoothing. Bochner-Riesz summability on $L^{p}$ describes the range of $\delta$ for which $S_{R}^{\delta}(L)$ are bounded on $L^{p}$, uniformly in $R$.

In Theorem 3.1, if one chooses $F(\lambda)=\left(1-\lambda^{2}\right)_{+}^{\delta}$ then $F \in W^{\beta, q}$ if and only if $\delta>\beta-1 / q$. Therefore, we obtain

Corollary 3.2. Suppose that the operator L satisfies the finite speed propagation property (FS) and condition $\left(\mathrm{ST}_{\mathrm{p}, \mathrm{s}}^{\mathrm{q}}\right)$ with some $1 \leq p<s \leq \infty$ and $1 \leq q \leq \infty$. Then for all $\delta>n(1 / p-1 / s)-1 / q$, we have

$$
\left\|\left(I-\frac{L}{R^{2}}\right)_{+}^{\delta}\right\|_{p \rightarrow p} \leq C
$$

uniformly in $R>0$.

As a consequence, we obtain the following necessary condition for the restriction condition $\left(\mathrm{ST}_{\mathrm{p}, \mathrm{s}}^{\mathrm{q}}\right)$ (see also Kenig, Stanton and Tomas [38]).

Corollary 3.3. Suppose that $1 / q>n(1 / p-1 / s)$ for some $q \geq 1$ and $1 \leq p<s \leq \infty$. Then condition $\left(\mathrm{ST}_{\mathrm{p}, \mathrm{s}}^{\mathrm{q}}\right)$ implies that $L=0$. 
Proof. Note that if $1 / q>n(1 / p-1 / s)$ for some $q \geq 1$ and $1 \leq p<s \leq \infty$, then there exist $\delta<0$ and $\varepsilon>0$ such that $S_{1}^{\delta}\left(\lambda^{2}\right)=\left(1-\lambda^{2}\right)_{+}^{\delta} \in W^{n\left(\frac{1}{p}-\frac{1}{s}\right)+\varepsilon, q}$. By Theorem 3.1, the operator $S_{R}^{\delta}(L)$ is bounded on $L^{p}(X)$ uniformly in $R$, i.e., $\left\|S_{R}^{\delta}(L)\right\|_{p \rightarrow p} \leq C<\infty$ for some constant $C>0$ independent of $R$. However, $S_{R}^{\delta}(L)$ is a self-adjoint operator, so $\left\|S_{R}^{\delta}(L)\right\|_{p^{\prime} \rightarrow p^{\prime}}<\infty$, and by interpolation, $\left\|S_{R}^{\delta}(L)\right\|_{2 \rightarrow 2}<\infty$. Let $M=1+\left\|S_{R}^{\delta}(L)\right\|_{2 \rightarrow 2}$. It follows that $S_{R}^{\delta}(\lambda)>M$ for all $\lambda \in\left[R^{2}\left(1-M^{1 / \delta}\right), R^{2}\right]$. By spectral theorem, this implies that $E_{L}\left[R^{2}\left(1-M^{1 / \delta}\right), R^{2}\right]=0$. As $R$ is arbitrary positive number, this implies $L=0$.

Remark 3.4. Note that condition $\left(\mathrm{ST}_{\mathrm{p}, \mathrm{s}}^{\mathrm{q}}\right)$ allows us to define the operator $S_{R}^{\delta}(L)$ even when $\delta<0$ when the function $\lambda \rightarrow S_{1}^{\delta}\left(\lambda^{2}\right)$ is unbounded.

We return to the discussion of Bochner-Riesz analysis in Section 5 and we now discuss a discreet version of Theorem 3.1.

It is not difficult to see that condition $\left(\mathrm{ST}_{\mathrm{p}, \mathrm{s}}^{\mathrm{q}}\right)$ with some $q<\infty$ implies that the set of point spectrum of $L$ is empty. Indeed, one has for all $0 \leq a<R$ and $x \in X$,

$$
\left\|1_{\{a\}}(\sqrt{L}) P_{B(x, r)}\right\|_{p \rightarrow s} \leq C V(x, r)^{\frac{1}{s}-\frac{1}{p}}(r R)^{n\left(\frac{1}{p}-\frac{1}{s}\right)}\left\|\mathbb{1}_{\{a\}}(R \cdot)\right\|_{q}=0, \quad R r \geq 1
$$

and therefore $\mathbb{1}_{\{a\}}(\sqrt{L})=0$. Due to $\sigma(L) \subseteq[0, \infty)$, it follows that the point spectrum of $L$ is empty. In particular, $\left(\mathrm{ST}_{\mathrm{p}, \mathrm{s}}^{\mathrm{q}}\right)$ cannot hold for any $q<\infty$ for elliptic operators on compact manifolds or for the harmonic oscillator. To be able to study these operators as well, we introduce a variation of condition $\left(\mathrm{ST}_{\mathrm{p}, \mathrm{s}}^{\mathrm{q}}\right)$. Following [21, 23], for an even Borel function $F$ with $\operatorname{supp} F \subseteq[-1,1]$ we define the norm $\|F\|_{N, q}$ by

$$
\|F\|_{N, q}=\left(\frac{1}{2 N} \sum_{\ell=1-N}^{N} \sup _{\lambda \in\left[\frac{\ell-1}{N}, \frac{\ell}{N}\right)}|F(\lambda)|^{q}\right)^{1 / q},
$$

where $q \in[1, \infty)$ and $N \in \mathbb{N}$. For $q=\infty$, we put $\|F\|_{N, \infty}=\|F\|_{\infty}$. It is obvious that $\|F\|_{N, q}$ increases monotonically in $q$.

Consider a non-negative self-adjoint operator $L$ and numbers $p, s$ and $q$ such that $1 \leq p<s \leq \infty$ and $1 \leq q \leq \infty$. We shall say that $L$ satisfies the Sogge spectral cluster condition if: for a fixed natural number $\kappa$ and for all $N \in \mathbb{N}$ and all even Borel functions $F$ such that $\operatorname{supp} F \subseteq[-N, N]$,

$$
\left\|F(\sqrt{L}) P_{B(x, r)}\right\|_{p \rightarrow s} \leq C V(x, r)^{\frac{1}{s}-\frac{1}{p}}(N r)^{n\left(\frac{1}{p}-\frac{1}{s}\right)}\left\|\delta_{N} F\right\|_{N^{\kappa}, q}
$$

for all $x \in X$ and $r \geq 1 / N$. For $q=\infty,\left(\mathrm{SC}_{\mathrm{p}, \mathrm{s}}^{\infty, \kappa}\right)$ is independent of $\kappa$ so we write it as $\left(\mathrm{SC}_{\mathrm{p}, \mathrm{s}}^{\infty}\right)$.

Remark 3.5. It is easy to check that for $\kappa \geq 1$, $\left(\mathrm{SC}_{\mathrm{p}, \mathrm{s}}^{\mathrm{q}, \kappa}\right)$ implies $\left(\mathrm{SC}_{\mathrm{p}, \mathrm{s}}^{\mathrm{q}, 1}\right)$.

Theorem 3.6. Suppose the operator L satisfies property (FS) and condition $\left(\mathrm{SC}_{\mathrm{p}, \mathrm{s}}^{\mathrm{q}, \kappa}\right)$ for a fixed $\kappa \in \mathbb{N}$ and some $p, s, q$ such that $1 \leq p<s \leq \infty$ and $1 \leq q \leq \infty$. In addition, we assume that for any $\varepsilon>0$ there exists a constant $C_{\varepsilon}$ such that for all $N \in \mathbb{N}$ and all even Borel functions $F$ such that supp $F \subset[-N, N]$,

$$
\|F(\sqrt{L})\|_{p \rightarrow p} \leq C_{\varepsilon} N^{\kappa n\left(\frac{1}{p}-\frac{1}{s}\right)+\varepsilon}\left\|\delta_{N} F\right\|_{N^{k}, q} .
$$

Then for any even function $F$ such that $\operatorname{supp} F \subseteq[-1,1]$ and $\|F\|_{W^{\beta, q}}<\infty$ for some $\beta>\max \{n(1 / p-$ $1 / s), 1 / q\}$, the operator $F(t \sqrt{L})$ is bounded on $L^{p}(X)$ for all $t>0$. In addition,

$$
\sup _{t>0}\|F(t \sqrt{L})\|_{p \rightarrow p} \leq C\|F\|_{W^{\beta, q}} .
$$


Note that condition $\left(\mathrm{SC}_{\mathrm{p}, \mathrm{s}}^{\mathrm{q}, \mathrm{s}}\right)$ is weaker than $\left(\mathrm{ST}_{\mathrm{p}, \mathrm{s}}^{\mathrm{q}}\right)$ and we need a priori estimate $\left(\mathrm{AB}_{\mathrm{p}}\right)$ in Theorem 3.6. See also [21, Theorem 3.6] and [23, Theorem 3.2] for related results. Once $\left(\mathrm{SC}_{\mathrm{p}, \mathrm{s}}^{\mathrm{q}, \kappa}\right)$ is proved, a priori estimate $\left(\mathrm{AB}_{\mathrm{p}}\right)$ is not difficult to check in general, see for example the section on the harmonic oscillator.

Following [23], we prove the following result.

Proposition 3.7. Suppose that $\mu(X)<\infty$ and $\left(\mathrm{SC}_{\mathrm{p}, \mathrm{s}}^{\mathrm{q}, 1}\right.$ ) for some $p, s, q$ such that $1 \leq p<s \leq \infty$ and $1 \leq q \leq \infty$. Then

$$
\|F(\sqrt{L})\|_{p \rightarrow p} \leq C N^{n\left(\frac{1}{p}-\frac{1}{s}\right)}\left\|\delta_{N} F\right\|_{N, q}
$$

for all $N \in \mathbb{N}$ and all Borel functions $F$ such that supp $F \subseteq[-N, N]$. Therefore, for any even function $F$ such that $\operatorname{supp} F \subseteq[-1,1]$ and $\|F\|_{W^{\beta, q}}<\infty$ for some $\beta>\max \{n(1 / p-1 / s), 1 / q\}$, the operator $F(t \sqrt{L})$ is bounded on $L^{p}(X)$ for all $t>0$ and

$$
\sup _{t>0}\|F(t \sqrt{L})\|_{p \rightarrow p} \leq C\|F\|_{W^{\beta, q}} .
$$

Proof. Since $\mu(X)<\infty$, we may assume that $X=B\left(x_{0}, 1\right)$ for some $x_{0} \in X$ (see [3]). It follows from Hölder's inequality and condition $\left(\mathrm{SC}_{\mathrm{p}, \mathrm{s}}^{\mathrm{q}, 1}\right)$ that

$$
\begin{aligned}
\|F(\sqrt{L})\|_{p \rightarrow p} & \leq V(X)^{\frac{1}{p}-\frac{1}{s}}\left\|F(\sqrt{L}) P_{B\left(x_{0}, 1\right)}\right\|_{p \rightarrow s} \\
& \leq C V(X)^{\frac{1}{p}-\frac{1}{s}} V(X)^{\frac{1}{s}-\frac{1}{p}} N^{n\left(\frac{1}{p}-\frac{1}{s}\right)}\left\|\delta_{N} F\right\|_{N, q} \\
& \leq C N^{n\left(\frac{1}{p}-\frac{1}{s}\right)}\left\|\delta_{N} F\right\|_{N, q} .
\end{aligned}
$$

This means that $\left(\mathrm{AB}_{\mathrm{p}}\right)$ is satisfied and thus the last assertion follows from Theorem 3.6. This proves Proposition 3.7.

The proof of Theorems 3.1 and 3.6 uses the following lemma. In the case where the volume is polynomial this lemma is proved in [28] using a similar argument.

Lemma 3.8. Suppose that $T$ is a linear map such that for all $x \in X$ and $r>0$ the operator $T P_{B(x, r)}$ is bounded from $L^{p}(X)$ to $L^{s}(X)$ for some $1 \leq p<s \leq \infty$. Assume also that

$$
\operatorname{supp} K_{T} \subseteq \mathcal{D}_{\rho}
$$

for some $\rho>0$. Then there exists a constant $C=C_{p, s}$ such that

$$
\|T\|_{p \rightarrow p} \leq C \sup _{x \in X}\left\{V(x, \rho)^{\frac{1}{p}-\frac{1}{s}}\left\|T P_{B(x, \rho)}\right\|_{p \rightarrow s}\right\} .
$$

Proof. We fix $\rho>0$. Then we choose a sequence $\left(x_{n}\right) \in X$ such that $d\left(x_{i}, x_{j}\right)>\rho / 10$ for $i \neq j$ and $\sup _{x \in X} \inf _{i} d\left(x, x_{i}\right) \leq \rho / 10$. Such sequence exists because $X$ is separable. Second we let $B_{i}=B\left(x_{i}, \rho\right)$ and define $\widetilde{B}_{i}$ by the formula

$$
\widetilde{B}_{i}=\bar{B}\left(x_{i}, \frac{\rho}{10}\right) \backslash \bigcup_{j<i} \bar{B}\left(x_{j}, \frac{\rho}{10}\right),
$$

where $\bar{B}(x, \rho)=\{y \in X: d(x, y) \leq \rho\}$. Third we put $\chi_{i}=\chi_{\widetilde{B}_{i}}$, where $\chi_{\widetilde{B}_{i}}$ is the characteristic function of the set $\widetilde{B}_{i}$. Note that for $i \neq j B\left(x_{i}, \frac{\rho}{20}\right) \cap B\left(x_{j}, \frac{\rho}{20}\right)=\emptyset$. Hence

$$
K=\sup _{i} \#\left\{j: d\left(x_{i}, x_{j}\right) \leq 2 \rho\right\} \leq \sup _{x} \frac{V\left(x,\left(2+\frac{1}{20}\right) \rho\right)}{V\left(x, \frac{\rho}{20}\right)}<C 41^{n}<\infty .
$$


It is not difficult to see that

so

$$
\mathcal{D}_{\rho} \subset \bigcup_{\left\{i, j: d\left(x_{i}, x_{j}\right)<2 \rho\right\}} \widetilde{B}_{i} \times \widetilde{B}_{j} \subset \mathcal{D}_{4 \rho}
$$

$$
T f=\sum_{i, j: d\left(x_{i}, x_{j}\right)<2 \rho} P_{\widetilde{B}_{i}} T P_{\widetilde{B}_{j}} f .
$$

Hence by Hölder's inequality

$$
\begin{array}{r}
\|T f\|_{p}^{p}=\left\|\sum_{i, j: d\left(x_{i}, x_{j}\right)<2 \rho} P_{\widetilde{B}_{i}} T P_{\widetilde{B}_{j}} f\right\|_{p}^{p}=\sum_{i}\left\|\sum_{j: d\left(x_{i}, x_{j}\right)<2 \rho} P_{\widetilde{B}_{i}} T P_{\widetilde{B}_{j}} f\right\|_{p}^{p} \\
\leq C K^{p-1} \sum_{i} \sum_{j: d\left(x_{i}, x_{j}\right)<2 \rho}\left\|P_{\widetilde{B}_{i}} T P_{\widetilde{B}_{j}} f\right\|_{p}^{p} \\
\leq C K^{p-1} \sum_{i} \sum_{j: d\left(x_{i}, x_{j}\right)<2 \rho} \mu\left(\widetilde{B}_{i}\right)^{p\left(\frac{1}{p}-\frac{1}{s}\right)}\left\|P_{\widetilde{B}_{i}} T P_{\widetilde{B}_{j}} f\right\|_{s}^{p} \\
\leq C K^{p} \sum_{j} \mu\left(B_{j}\right)^{p\left(\frac{1}{p}-\frac{1}{s}\right)}\left\|T P_{\widetilde{B}_{j}} f\right\|_{s}^{p} \\
\leq C K^{p} \sum_{j} \mu\left(B_{j}\right)^{p\left(\frac{1}{p}-\frac{1}{s}\right)}\left\|T P_{\widetilde{B}_{j}}\right\|_{p \rightarrow s}^{p}\left\|P_{\widetilde{B}_{j}} f\right\|_{p}^{p} \\
\leq C K^{p} \sup _{x \in X}\left\{V(x, \rho)^{p\left(\frac{1}{p}-\frac{1}{s}\right)}\left\|T P_{B(x, \rho)}\right\|_{p \rightarrow s}^{p}\right\} \sum_{j}\left\|P_{\widetilde{B}_{j}} f\right\|_{p}^{p} \\
=C K^{p} \sup _{x \in X}\left\{V(x, \rho)^{p\left(\frac{1}{p}-\frac{1}{s}\right)}\left\|T P_{B(x, \rho)}\right\|_{p \rightarrow s}^{p}\right\}\|f\|_{p}^{p} .
\end{array}
$$

This finishes the proof of Lemma 3.8.

Proof of Theorem 3.1. Let $\eta \in C_{c}^{\infty}(\mathbb{R})$ be even and such that $\operatorname{supp} \eta \subseteq\{\xi: 1 / 4 \leq|\xi| \leq 1\}$ and

$$
\sum_{\ell \in \mathbb{Z}} \eta\left(2^{-\ell} \lambda\right)=1 \quad \forall \lambda>0
$$

Then we set $\eta_{0}(\lambda)=1-\sum_{\ell>0} \eta\left(2^{-\ell} \lambda\right)$,

$$
F^{(0)}(\lambda)=\frac{1}{2 \pi} \int_{-\infty}^{+\infty} \eta_{0}(t) \hat{F}(t) \cos (t \lambda) d t
$$

and

$$
F^{(\ell)}(\lambda)=\frac{1}{2 \pi} \int_{-\infty}^{+\infty} \eta\left(2^{-\ell} t\right) \hat{F}(t) \cos (t \lambda) d t .
$$

Note that in virtue of the Fourier inversion formula

$$
F(\lambda)=\sum_{\ell \geq 0} F^{(\ell)}(\lambda)
$$

and by Lemma 2.1

$$
\operatorname{supp} K_{F^{(\ell)}(t \sqrt{L})} \subset \mathcal{D}_{2^{\ell} t} .
$$

Now by Lemma 3.8

$$
\begin{aligned}
\|F(t \sqrt{L})\|_{p \rightarrow p} & \leq \sum_{\ell \geq 0}\left\|F^{(\ell)}(t \sqrt{L})\right\|_{p \rightarrow p} \\
& \leq \sum_{\ell \geq 0} \sup _{x \in X}\left\{V\left(x, 2^{\ell} t\right)^{\frac{1}{p}-\frac{1}{s}}\left\|F^{(\ell)}(t \sqrt{L}) P_{B\left(x, 2^{\ell} t\right)}\right\|_{p \rightarrow s}\right\} .
\end{aligned}
$$


Since $F^{(\ell)}$ is not compactly supported we choose a function $\psi \in C_{c}^{\infty}(-4,4)$ such that $\psi(\lambda)=1$ for $\lambda \in(-2,2)$ and note that

$$
\begin{aligned}
& \left\|F^{(\ell)}(t \sqrt{L}) P_{B\left(x, 2^{\ell} t\right)}\right\|_{p \rightarrow s} \\
& \quad \leq\left\|\left(\psi F^{(\ell)}\right)(t \sqrt{L}) P_{B\left(x, 2^{\ell} t\right)}\right\|_{p \rightarrow s}+\left\|\left((1-\psi) F^{(\ell)}\right)(t \sqrt{L}) P_{B\left(x, 2^{\ell} t\right)}\right\|_{p \rightarrow s} .
\end{aligned}
$$

To estimate the norm $\left\|\left(\psi F^{(\ell)}\right)(t \sqrt{L}) P_{B\left(x, 2^{\ell} t\right)}\right\|_{p \rightarrow s}$, we use condition $\left(\mathrm{ST}_{\mathrm{p}, \mathrm{s}}^{\mathrm{q}}\right)$ and the fact that $\psi \in$ $C_{c}(-4,4)$ to obtain

$$
\left\|\left(\psi F^{(\ell)}\right)(t \sqrt{L}) P_{B\left(x, 2^{\ell} t\right)}\right\|_{p \rightarrow s} \leq C V\left(x, 2^{\ell} t\right)^{\frac{1}{s}-\frac{1}{p}} 2^{\ln \left(\frac{1}{p}-\frac{1}{s}\right)}\left\|\delta_{4 t^{-1}}\left(\psi F^{(\ell)}\right)(t \cdot)\right\|_{q}
$$

for all $t>0$. Hence

$$
\begin{aligned}
\sum_{\ell \geq 0} \sup _{x \in X}\left\{V\left(x, 2^{\ell} t\right)^{\frac{1}{p}-\frac{1}{s}}\left\|\left(\psi F^{(\ell)}\right)(t \sqrt{L}) P_{B\left(x, 2^{\ell}\right)}\right\|_{p \rightarrow s}\right\} & \leq C \sum_{\ell \geq 0} 2^{\ln \left(\frac{1}{p}-\frac{1}{s}\right)}\left\|\delta_{4 t^{-1}}\left(\psi F^{(\ell)}\right)(t \cdot)\right\|_{q} \\
& \leq C \sum_{\ell \geq 0} 2^{\ln \left(\frac{1}{p}-\frac{1}{s}\right)}\left\|F^{(\ell)}\right\|_{q} \\
& =C\|F\|_{B_{q, 1}^{n\left(\frac{1}{p}-\frac{1}{s}\right)}}
\end{aligned}
$$

where the last equality follows from definition of Besov space. See, e.g., [5, Chap. VI ]. Recall also that if $\beta>n(1 / p-1 / s)$ then $W^{\beta, q} \subseteq B_{q, 1}^{n(1 / p-1 / s)}$ and $\|F\|_{B_{q, 1}^{n(1 / p-1 / s)}} \leq C_{\beta}\|F\|_{W^{\beta, q}}$, see again [5]. Hence the forgoing estimates give

$$
\sum_{\ell \geq 0} \sup _{x \in X}\left\{V\left(x, 2^{\ell} t\right)^{\frac{1}{p}-\frac{1}{s}}\left\|\left(\psi F^{(\ell)}\right)(t \sqrt{L}) P_{B\left(x, 2^{\ell} t\right)}\right\|_{p \rightarrow s}\right\} \leq C\|F\|_{W^{\beta, q}} .
$$

Next we show bounds for $\left\|\left((1-\psi) F^{(\ell)}\right)(t \sqrt{L}) P_{B\left(x, 2^{\ell} t\right)}\right\|_{p \rightarrow s}$. Since the function $1-\psi$ is supported outside the interval $(-2,2)$, we can choose a function $\phi \in C_{c}^{\infty}(2,8)$ such that

$$
1=\psi(\lambda)+\sum_{k \geq 0} \phi\left(2^{-k} \lambda\right)=\psi(\lambda)+\sum_{k \geq 0} \phi_{k}(\lambda) \quad \forall \lambda>0 .
$$

Hence

$$
\left((1-\psi) F^{(\ell)}\right)(\lambda)=\sum_{k \geq 0}\left(\phi_{k} F^{(\ell)}\right)(\lambda) \quad \forall \lambda>0 .
$$

It follows from the implication $\left(\mathrm{ST}_{\mathrm{p}, \mathrm{s}}^{\mathrm{q}}\right) \Rightarrow\left(\mathrm{ST}_{\mathrm{p}, \mathrm{s}}^{\infty}\right)$ that

$$
\begin{aligned}
\left\|\left((1-\psi) F^{(\ell)}\right)(t \sqrt{L}) P_{B\left(x, 2^{\ell} t\right)}\right\|_{p \rightarrow s} & \leq \sum_{k \geq 0}\left\|\left(\phi_{k} F^{(\ell)}\right)(t \sqrt{L}) P_{B\left(x, 2^{\ell} t\right)}\right\|_{p \rightarrow s} \\
& \leq C \sum_{k \geq 0} V\left(x, 2^{\ell} t\right)^{\frac{1}{s}-\frac{1}{p}} 2^{n(\ell+k)\left(\frac{1}{p}-\frac{1}{s}\right)}\left\|\delta_{2^{k+3} t^{-1}}\left(\phi_{k} F^{(\ell)}\right)(t \cdot)\right\|_{\infty} .
\end{aligned}
$$

Note that $\operatorname{supp} F \in[-1,1], \operatorname{supp} \phi \subset[2,8]$ and $\check{\eta}$ is in the Schwartz class so

$$
\left\|\phi_{k} F^{(\ell)}\right\|_{\infty}=2^{l}\left\|\phi_{k}\left(F * \delta_{2^{i}} \check{\eta}\right)\right\|_{\infty} \leq C 2^{-M(\ell+k)}\|F\|_{q}
$$

and similarly, $\left\|\phi_{k} F^{(0)}\right\|_{\infty} \leq C 2^{-M k}\|F\|_{q}$. Therefore

$$
\begin{aligned}
\left\|\left((1-\psi) F^{(\ell)}\right)(t \sqrt{L}) P_{B\left(x, 2^{\ell} t\right)}\right\|_{p \rightarrow s} & \leq C \sum_{k \geq 0} V\left(x, 2^{\ell} t\right)^{\frac{1}{s}-\frac{1}{p}} 2^{n(\ell+k)\left(\frac{1}{p}-\frac{1}{s}\right)} 2^{-M(\ell+k)}\|F\|_{q} \\
& \leq C V\left(x, 2^{\ell} t\right)^{\frac{1}{s}-\frac{1}{p}} 2^{\ell\left(n\left(\frac{1}{p}-\frac{1}{s}\right)-M\right)}\|F\|_{q} .
\end{aligned}
$$


Hence

$$
\begin{aligned}
\sum_{\ell \geq 0} \sup _{x \in X}\left\{V\left(x, 2^{\ell} t\right)^{\frac{1}{p}-\frac{1}{s}}\left\|\left((1-\psi) F^{(\ell)}\right)(t \sqrt{L}) P_{B\left(x, 2^{\ell} t\right)}\right\|_{p \rightarrow s}\right\} & \leq C \sum_{\ell \geq 0} 2^{\ell\left(n\left(\frac{1}{p}-\frac{1}{s}\right)-M\right)}\|F\|_{q} \\
& \leq C\|F\|_{q} .
\end{aligned}
$$

Now estimate (3.1) follows from (3.12), (3.7), (3.8) and (3.10). This completes the proof of Theorem 3.1.

\section{Proof of Theorem 3.6.}

Case (1). $t \geq 1 / 4$.

If $t \geq 1 / 4$ then $\operatorname{supp} \delta_{t} F \subset[-4,4]$. By $\left(\mathrm{AB}_{\mathrm{p}}\right)$,

$$
\|F(t \sqrt{L})\|_{p \rightarrow p} \leq C 4^{\kappa n\left(\frac{1}{p}-\frac{1}{s}\right)+\varepsilon}\left\|\delta_{4}(F(t \cdot))\right\|_{4^{\kappa}, q} \leq C\|F\|_{\infty} .
$$

Recall that if $\beta>1 / q$, then $W^{\beta, q}(\mathbb{R}) \subseteq L^{\infty}(\mathbb{R}) \cap C(\mathbb{R})$ and $\|F\|_{\infty} \leq C\|F\|_{W^{\beta, q}}$. Hence

$$
\sup _{t \geq 1 / 4}\|F(t \sqrt{L})\|_{p \rightarrow p} \leq C\|F\|_{\infty} \leq C\|F\|_{W^{\beta, q}} .
$$

Case (2). $t \leq 1 / 4$.

Let $\xi \in C_{c}^{\infty}$ be an even function such that $\operatorname{supp} \xi \subset[-1,1], \hat{\xi}(0)=1$ and $\hat{\xi}^{(k)}(0)=0$ for all $1 \leq k \leq[\beta]+2$. Write $\xi_{N^{k-1}}=N^{\kappa-1} \xi\left(N^{\kappa-1} \cdot\right)$ where $N=8\left[t^{-1}\right]+1$ and $\left[t^{-1}\right]$ denotes the integer part of $t^{-1}$. Following [21] we write

$$
F(t \sqrt{L})=\left(\delta_{t} F-\xi_{N^{k-1}} * \delta_{t} F\right)(\sqrt{L})+\left(\xi_{N^{k-1}} * \delta_{t} F\right)(\sqrt{L}) .
$$

We first prove that

$$
\left\|\left(\delta_{t} F-\xi_{N^{\kappa-1}} * \delta_{t} F\right)(\sqrt{L})\right\|_{p \rightarrow p} \leq C\|F\|_{W^{\beta, q}} .
$$

Observe that $\operatorname{supp}\left(\delta_{t} F-\xi_{N^{\kappa-1}} * \delta_{t} F\right) \subseteq[-N, N]$. We apply $\left(\mathrm{AB}_{\mathrm{p}}\right)$ to obtain

$$
\left\|\left(\delta_{t} F-\xi_{N^{\kappa-1}} * \delta_{t} F\right)(\sqrt{L})\right\|_{p \rightarrow p} \leq C N^{\kappa n\left(\frac{1}{p}-\frac{1}{s}\right)+\varepsilon}\left\|\delta_{N}\left(\delta_{t} F-\xi_{N^{\kappa-1}} * \delta_{t} F\right)\right\|_{N^{k}, q} .
$$

Everything then boils down to estimate $\|\cdot\|_{N^{k}, q}$ norm of $\delta_{N}\left(\delta_{t} F-\xi_{N^{k-1}} * \delta_{t} F\right)$. We make the following claim. For its proof, see [21, (3.29)] or [23, Propostion 4.6].

Lemma 3.9. Suppose that $\xi \in C_{c}^{\infty}$ is an even function such that $\operatorname{supp} \xi \subset[-1,1], \hat{\xi}(0)=1$ and $\hat{\xi}^{(k)}(0)=0$ for all $1 \leq k \leq[\beta]+2$. Next assume that $\operatorname{supp} H \subset[-1,1]$. Then

$$
\left\|H-\xi_{N} * H\right\|_{N, q} \leq C N^{-\beta}\|H\|_{W^{\beta, q}}
$$

for all $\beta>1 / q$ and any $N \in \mathbb{N}$.

Note that $\delta_{N}\left(\delta_{t} F-\xi_{N^{k-1}} * \delta_{t} F\right)=\delta_{N t} F-\xi_{N^{k}} * \delta_{N t} F$. Now, if $\beta>\max \{n(1 / p-1 / s), 1 / q\}$ then (3.13) follows from Lemma 3.9 and estimate (3.14).

It remains to show that

$$
\left\|\left(\xi_{N^{\kappa-1}} * \delta_{t} F\right)(\sqrt{L})\right\|_{p \rightarrow p} \leq C\|F\|_{W^{\beta, q}} .
$$

Let $F^{(\ell)}$ be functions defined in (3.5) and (3.6). Following the proof of Theorem 3.1, we write

$$
\left(\xi_{N^{k-1}} * \delta_{t} F\right)(\lambda)=\sum_{\ell \geq 0}\left(\xi_{N^{k-1}} * \delta_{t} F^{(\ell)}\right)(\lambda),
$$


and by Lemma 2.1, $\operatorname{supp} K_{\left(\xi_{N^{k-1}} * \delta_{t} F^{(\ell)}\right)(\sqrt{L})} \subset \mathcal{D}_{2^{\ell}{ }_{t}}$. Now by Lemma 3.8

$$
\begin{aligned}
\left\|\left(\xi_{N^{k-1}} * \delta_{t} F\right)(\sqrt{L})\right\|_{p \rightarrow p} & \leq \sum_{\ell \geq 0}\left\|\left(\xi_{N^{k-1}} * \delta_{t} F^{(\ell)}\right)(\sqrt{L})\right\|_{p \rightarrow p} \\
& \leq \sum_{\ell \geq 0} \sup _{x \in X}\left\{V\left(x, 2^{\ell} t\right)^{\frac{1}{p}-\frac{1}{s}}\left\|\left(\xi_{N^{k-1}} * \delta_{t} F^{(\ell)}\right)(\sqrt{L}) P_{B\left(x, 2^{\ell} t\right)}\right\|_{p \rightarrow s}\right\} .
\end{aligned}
$$

Take a function $\psi \in C_{c}^{\infty}(-4,4)$ such that $\psi(\lambda)=1$ for $\lambda \in(-2,2)$. Then

$\left\|\left(\xi_{N^{\kappa-1}} * \delta_{t} F^{(\ell)}\right)(\sqrt{L}) P_{B\left(x, 2^{\ell} t\right)}\right\|_{p \rightarrow s} \leq\left\|\left(\delta_{t} \psi\left(\xi_{N^{\kappa-1}} * \delta_{t} F^{(\ell)}\right)\right)(\sqrt{L}) P_{B\left(x, 2^{\ell} t\right)}\right\|_{p \rightarrow s}$

Note that $\left(\mathrm{SC}_{\mathrm{p}, \mathrm{s}}^{\mathrm{q}, K}\right) \Rightarrow\left(\mathrm{SC}_{\mathrm{p}, \mathrm{s}}^{\infty}\right)$ and $t \leq 1 / 4$. Using $\left(\mathrm{SC}_{\mathrm{p}, \mathrm{s}}^{\infty}\right)$ instead of $\left(\mathrm{ST}_{\mathrm{p}, \mathrm{s}}^{\infty}\right)$, we show as in the proof of (3.11) that $I_{\ell} \leq C V\left(x, 2^{\ell} t\right)^{\frac{1}{s}-\frac{1}{p}} 2^{\ell\left(n\left(\frac{1}{p}-\frac{1}{s}\right)-M\right)}\|F\|_{q}$ for some large $M>n(1 / p-1 / s)+1$.

Next we estimate the term $I_{\ell}$. We assume that $\psi \in C_{c}(-4,4)$ so by $\left(\mathrm{SC}_{\mathrm{p}, \mathrm{s}}^{\mathrm{q}, K}\right)$

$$
I_{\ell} \leq C V\left(x, 2^{\ell} t\right)^{\frac{1}{s}-\frac{1}{p}} 2^{\ell n\left(\frac{1}{p}-\frac{1}{s}\right)}\left\|\delta_{N}\left(\delta_{t} \psi\left(\xi_{N^{\kappa-1}} * \delta_{t} F^{(\ell)}\right)\right)\right\|_{N^{\kappa}, q^{*}} .
$$

Observe that (see also [21, (3.19)])

So

$$
\left|\left(\xi * \delta_{t} F^{(\ell)}\right)(\lambda)\right| \leq\|\xi\|_{L^{q^{\prime}}}\left(\int_{\lambda-1}^{\lambda+1}\left|F^{(\ell)}(t u)\right|^{q} d u\right)^{1 / q}
$$

$$
\begin{aligned}
\left\|\delta_{N}\left(\delta_{t} \psi\left(\xi_{N^{\kappa-1}} * \delta_{t} F^{(\ell)}\right)\right)\right\|_{N^{\kappa}, q} & =\left(\frac{1}{2 N^{\kappa}} \sum_{i=1-N^{\kappa}}^{N^{\kappa}} \sup _{\lambda \in\left[\frac{i-1}{N^{\kappa}}, \frac{i}{N^{\kappa}}\right)}\left|\psi(t N \lambda)\left(\xi_{N^{\kappa-1}} * \delta_{t} F^{(\ell)}\right)(N \lambda)\right|^{q}\right)^{1 / q} \\
& \leq C\left(\frac{1}{N^{\kappa}} \sum_{i=1-N^{\kappa}}^{N^{\kappa}} \sup _{\lambda \in[i-1, i)}\left|\left(\xi * \delta_{t N^{1-\kappa}} F^{(\ell)}\right)(\lambda)\right|^{q}\right)^{1 / q} \\
& \leq C\|\xi\|_{q^{\prime}}\left(\frac{1}{N^{\kappa}} \sum_{i=1-N^{\kappa}}^{N^{\kappa}} \sup _{\lambda \in[i-1, i)} \int_{\lambda-1}^{\lambda+1}\left|F^{(\ell)}\left(t N^{1-\kappa} u\right)\right|^{q} d u\right)^{1 / q} \\
& \leq C\left(\frac{1}{N^{\kappa}} \sum_{i=1-N^{\kappa}}^{N^{\kappa}} \int_{i-2}^{i+1}\left|F^{(\ell)}\left(t N^{1-\kappa} u\right)\right|^{q} d u\right)^{1 / q} \\
& \leq C\left(\frac{1}{N t} \int_{-\infty}^{\infty}\left|F^{(\ell)}(u)\right|^{q} d u\right)^{1 / q} \leq C\left\|F^{(\ell)}\right\|_{q} .
\end{aligned}
$$

This shows that $I_{\ell} \leq C V\left(x, 2^{\ell} t\right)^{\frac{1}{s}-\frac{1}{p}} 2^{\ln \left(\frac{1}{p}-\frac{1}{s}\right)}\left\|F^{(\ell)}\right\|_{q}$. Using the above estimates of $I_{\ell}$ and $I_{\ell}$, together with (3.17) and (3.18), we can argue as in (3.10) and (3.12) to obtain estimate (3.16). This proves Theorem 3.6.

Now we discuss another condition introduced by C.D. Sogge (see $[56,57,58]$ ). We say that $L$ satisfies $\left(p, p^{\prime}\right)$ spectral cluster estimate $\left(\mathrm{S}_{\mathrm{p}}\right)$ for some $1 \leq p<2$ and its conjugate $p^{\prime}$ if the spectral projection $E_{\sqrt{L}}[k, k+1)$ maps $L^{p}(X)$ to $L^{p^{\prime}}(X)$ and

$$
\left\|E_{\sqrt{L}}[k, k+1)\right\|_{p \rightarrow p^{\prime}} \leq C(1+k)^{n\left(\frac{1}{p}-\frac{1}{p^{\prime}}\right)-1}
$$

for all $k \geq 0$.

Proposition 3.10. Suppose that $1 \leq p \leq 2 n /(n+1), \mu(X)<\infty$ and $V(x, r) \leq C \min \left(r^{n}, 1\right)$ for every $x \in X$ and $r>0$. Then conditions $\left(\mathrm{S}_{\mathrm{p}}\right)$ and $\left(\mathrm{SC}_{\mathrm{p}, 2}^{2,1}\right)$ are equivalent. 
Proof. We first prove the implication $\left(\mathrm{S}_{\mathrm{p}}\right) \Rightarrow\left(\mathrm{SC}_{\mathrm{p}, 2}^{2,1}\right)$. Note that for every even Borel function $F$ such that $\operatorname{supp} F \subset[-N, N]$,

$$
\begin{aligned}
\|F(\sqrt{L}) f\|_{2}^{2} & =\sum_{k=0}^{N}\left\langle E_{\sqrt{L}}[k, k+1) F(\sqrt{L}) f, E_{\sqrt{L}}[k, k+1) F(\sqrt{L}) f\right\rangle \\
& \leq \sum_{k=0}^{N}\left\|E_{\sqrt{L}}[k, k+1) F(\sqrt{L})\right\|_{p \rightarrow 2}^{2}\|f\|_{p}^{2} .
\end{aligned}
$$

Using a $T^{*} T$ argument and condition $\left(\mathrm{S}_{\mathrm{p}}\right)$ we obtain

$$
\begin{aligned}
\left\|F(\sqrt{L}) P_{B(x, r)}\right\|_{p \rightarrow 2}^{2} & \leq C\|F(\sqrt{L})\|_{p \rightarrow 2}^{2} \\
& \leq C \sum_{k=0}^{N}\left\|E_{\sqrt{L}}[k, k+1) F(\sqrt{L})\right\|_{p \rightarrow 2}^{2} \\
& \leq C \sum_{k=0}^{N} \sup _{\lambda \in[k, k+1)}|F(\lambda)|^{2}(1+k)^{n\left(\frac{1}{p}-\frac{1}{p^{\prime}}\right)-1} \\
& \leq C N^{n\left(\frac{1}{p}-\frac{1}{p^{\prime}}\right)} \frac{1}{N} \sum_{k=0}^{N} \sup _{\lambda \in[k, k+1)}|F(\lambda)|^{2} \\
& \leq C V(x, r)^{2\left(\frac{1}{2}-\frac{1}{p}\right)}(N r)^{2 n\left(\frac{1}{p}-\frac{1}{2}\right)}\left\|\delta_{N} F\right\|_{N, 2}^{2},
\end{aligned}
$$

and hence condition $\left(\mathrm{SC}_{\mathrm{p}, 2}^{2,1}\right)$ is satisfied.

Next we prove the implication $\left(\mathrm{SC}_{\mathrm{p}, 2}^{2,1}\right) \Rightarrow\left(\mathrm{S}_{\mathrm{p}}\right)$. By $\left(\mathrm{SC}_{\mathrm{p}, 2}^{2,1}\right)$

$$
\left\|\chi_{[k, k+1)}(\sqrt{L})\right\|_{p \rightarrow 2} \leq C(1+k)^{n\left(\frac{1}{p}-\frac{1}{2}\right)}\left\|\delta_{(1+k)} \chi_{[k, k+1)}\right\|_{1+k, 2}
$$

Hence

$$
\begin{aligned}
\left\|E_{\sqrt{L}}[k, k+1)\right\|_{p \rightarrow p^{\prime}} & =\left\|E_{\sqrt{L}}[k, k+1)\right\|_{p \rightarrow 2}^{2} \\
& \leq C(1+k)^{2 n\left(\frac{1}{p}-\frac{1}{2}\right)}\left\|\delta_{(1+k)} \chi_{[k, k+1)}\right\|_{1+k, 2}^{2} \\
& \leq C(k+1)^{n\left(\frac{1}{p}-\frac{1}{p^{\prime}}\right)-1},
\end{aligned}
$$

which shows $\left(\mathrm{S}_{\mathrm{p}}\right)$.

Proposition 3.11. Assume that $\mu(X)<\infty$. Then conditions $\left(\mathrm{SC}_{\mathrm{p}, 2}^{\infty}\right)$ and $\left(\mathrm{ST}_{\mathrm{p}, 2}^{\infty}\right)$ are equivalent.

Proof. Since $\mu(X)<\infty$, we may assume that $X=B\left(x_{0}, 1\right)$ for some $x_{0} \in X$. Observing that $\|F\|_{N, \infty}=\|F\|_{\infty}$, we have the implication $\left(\mathrm{ST}_{\mathrm{p}, 2}^{\infty}\right) \Rightarrow\left(\mathrm{SC}_{\mathrm{p}, 2}^{\infty}\right)$. Let us show the implication $\left(\mathrm{SC}_{\mathrm{p}, 2}^{\infty}\right) \Rightarrow$ $\left(\mathrm{ST}_{\mathrm{p}, 2}^{\infty}\right)$. Assume that supp $F \subseteq[0, R]$. If $R \geq 1$, then we let $N=[R]+1$ and $\left(\mathrm{ST}_{\mathrm{p}, 2}^{\infty}\right)$ follows readily. Now for $0<R<1$, from condition $\left(\mathrm{SC}_{\mathrm{p}, 2}^{\infty}\right)$ we can take $N=1$ and the ball $B\left(x_{0}, 1\right)$ to obtain

$$
\|F(\sqrt{L})\|_{p \rightarrow 2} \leq C\|F\|_{\infty} .
$$

Now for any $x \in X$ and $r>0$, we note that conditions $1 / 2-1 / p<0$ and $\mu(X)<\infty$ give that $V(x, r)^{1 / 2-1 / p} \geq C$. Hence for any $r \geq 1 / R$,

$$
\left\|F(\sqrt{L}) P_{B(x, r)}\right\|_{p \rightarrow 2} \leq C\|F\|_{\infty} \leq C V(x, r)^{\frac{1}{2}-\frac{1}{p}}(r R)^{n\left(\frac{1}{p}-\frac{1}{2}\right)}\left\|\delta_{R} F\right\|_{\infty},
$$

this is $\left(\mathrm{ST}_{\mathrm{p}, 2}^{\infty}\right)$. The proof of Proposition 3.11 is finished. 


\section{SharP SPECTRAL MULTIPLIERS - SINGULAR INTEGRAL CASE}

4.1. Statements. As in Section 3 we discuss two type of results corresponding to estimates $\left(\mathrm{ST}_{\mathrm{p}, 2}^{\mathrm{q}}\right)$ or $\left(\mathrm{SC}_{\mathrm{p}, 2}^{\mathrm{q}, \kappa}\right)$. The aim of this section is to prove singular integral versions of Theorems 3.1 and 3.6. We use the same assumptions and notation as in Section 3. Recall that $n$ is a homogeneous dimension from (2.2). Fix a non-trivial auxiliary function $\eta \in C_{c}^{\infty}(0, \infty)$.

Theorem 4.1. Assume that operator $L$ satisfies property $(\mathrm{FS})$ and condition $\left(\mathrm{ST}_{\mathrm{p}, 2}^{\mathrm{q}}\right)$ for some $p, q$ satisfying $1 \leq p<2$ and $1 \leq q \leq \infty$. Then for any even bounded Borel function $F$ such that $\sup _{t>0}\left\|\eta \delta_{t} F\right\|_{W^{\beta, q}}<\infty$ for some $\beta>\max \{n(1 / p-1 / 2), 1 / q\}$ the operator $F(\sqrt{L})$ is bounded on $L^{r}(X)$ for all $p<r<p^{\prime}$. In addition,

$$
\|F(\sqrt{L})\|_{r \rightarrow r} \leq C_{\beta}\left(\sup _{t>0}\left\|\eta \delta_{t} F\right\|_{W^{\beta, q}}+|F(0)|\right) .
$$

Note that if $q<\infty$ then condition $\left(\mathrm{ST}_{\mathrm{p}, 2}^{\mathrm{q}}\right)$ implies that $E_{L}(\{0\})=0$ (and in fact $E_{L}(\{\lambda\})=0$ ) so the term $F(0)$ can be omitted in the above statement.

The next theorem is a variation of Theorem 4.1 suitable for the operators satisfying condition $\left(\mathrm{SC}_{\mathrm{p}, 2}^{\mathrm{q}, \kappa}\right)$. It is a singular integral version of Theorem 3.6 above.

Theorem 4.2. Suppose the operator $L$ satisfies property $(\mathrm{FS})$, conditions $\left(\mathrm{E}_{\mathrm{p}, 2}\right)$ and $\left(\mathrm{SC}_{\mathrm{p}, 2}^{\mathrm{q}, K}\right)$ for some $p, q$ such that $1 \leq p<2$ and $1 \leq q \leq \infty$, and a fixed natural number $\kappa$. In addition, we assume that for any $\varepsilon>0$ there exists a constant $C_{\varepsilon}$ such that for all $N \in \mathbb{N}$ and all even Borel functions $F$ such that supp $F \subset[-N, N]$,
$\left(\mathrm{AB}_{\mathrm{p}}\right)$$$
\|F(\sqrt{L})\|_{p \rightarrow p} \leq C_{\varepsilon} N^{\kappa n\left(\frac{1}{p}-\frac{1}{2}\right)+\varepsilon}\left\|\delta_{N} F\right\|_{N^{\kappa}, q} .
$$

Then for any even bounded Borel function $F$ such that $\sup _{t>0}\left\|\eta \delta_{t} F\right\|_{W^{\beta, q}}<\infty$ for some $\beta>$ $\max \{n(1 / p-1 / 2), 1 / q\}$ the operator $F(\sqrt{L})$ is bounded on $L^{r}(X)$ for all $p<r<p^{\prime}$. In addition,

$$
\|F(\sqrt{L})\|_{r \rightarrow r} \leq C_{\beta}\left(\sup _{t>0}\left\|\eta \delta_{t} F\right\|_{W^{\beta, q}}+|F(0)|\right) .
$$

Remark 4.3. Suppose that $\mu(X)<\infty$ and $\left(\mathrm{SC}_{\mathrm{p}, 2}^{\mathrm{q}, \kappa}\right)$ holds for some $\kappa \geq 1$. Then $\left(\mathrm{SC}_{\mathrm{p}, 2}^{\infty}\right)$ and $\left(\mathrm{E}_{\mathrm{p}, 2}\right)$ are satisfied by Remark 3.5 and Proposition 3.11. In addition, $\left(\mathrm{AB}_{\mathrm{p}}\right)$ holds by Proposition 3.7. Therefore, Theorem 4.2 holds in this case without assumptions $\left(\mathrm{E}_{\mathrm{p}, 2}\right)$ and $\left(\mathrm{AB}_{\mathrm{p}}\right)$.

By a classical dyadic decomposition of $F$, we can write $F(\sqrt{L})$ as the sum $\sum F_{j}(\sqrt{L})$. Then we apply Theorems 3.1 and 3.6 to estimate $\left\|F_{j}(\sqrt{L})\right\|_{r \rightarrow r}$. However, as mentioned in the introduction, this does not automatically imply that the operator $F(\sqrt{L})$ acts boundedly on $L^{r}$. See $[11,52,53]$ where this problem is discussed in the Euclidean case. Our proof is almost identical to one in [11]. Nevertheless we give full details because the changes which are required to adapt the arguments to the general setting are not trivial.

Note that condition $\left(\mathrm{ST}_{\mathrm{p}, 2}^{\mathrm{q}}\right)$ implies $\left(\mathrm{E}_{\mathrm{p}, 2}\right)$ (see Proposition 2.3). Therefore Theorems 4.1 and 4.2 follow from Theorems 3.1 and 3.6 (with $s=2$ ) and the next result.

Theorem 4.4. Assume that L satisfies the finite speed propagation property (FS) and condition $\left(\mathrm{E}_{\mathrm{p}_{0}, 2}\right)$ for some $1 \leq p_{0}<2$. Next assume that for all even Borel functions $F$ such that $\operatorname{supp} F$ is compact and $\|F\|_{W^{\beta, q}}<\infty$ for some $\beta$ and $q$ satisfying $\beta>\max \left\{n\left(1 / p_{0}-1 / 2\right), 1 / q\right\}$ and $1 \leq q \leq \infty$,

$$
\sup _{t>0}\|F(t \sqrt{L})\|_{p \rightarrow p} \leq C\|F\|_{W^{\beta, q}}, \quad p_{0} \leq p \leq p_{0}^{\prime} .
$$


Then for any bounded Borel function $F$ such that

$$
\sup _{t>0}\left\|\eta \delta_{t} F\right\|_{W^{\beta, q}}<\infty
$$

for some $\beta>\max \left\{n\left(1 / p_{0}-1 / 2\right), 1 / q\right\}$, the operator $F(\sqrt{L})$ is bounded on $L^{r}(X)$ for all $p_{0}<r<p_{0}^{\prime}$.

4.2. Singular integrals. This subsection is devoted to the proof of Theorem 4.4. We start with the following lemma.

Lemma 4.5. Suppose that operator L satisfies property $(\mathrm{FS})$ and condition $\left(\mathrm{E}_{\mathrm{p}_{0}, 2}\right)$ for some $1 \leq$ $p_{0}<2$.

(a) Assume in addition that $F$ is an even bounded Borel function such that

$$
\sup _{t>0}\left\|\eta \delta_{t} F\right\|_{C^{k}}<\infty
$$

for some integer $k>n / 2+1$ and some non-trivial function $\eta \in C_{c}^{\infty}(0, \infty)$. Then the operator $F(\sqrt{L})$ is bounded on $L^{p}(X)$ for all $p_{0}<p<p_{0}^{\prime}$.

(b) Assume in addition that $\psi$ be an even function in $\mathscr{S}(\mathbb{R})$ such that $\psi(0)=0$. Define the quadratic functional for $f \in L^{2}(X)$

$$
\mathcal{G}_{L}(f)(x)=\left(\sum_{j \in \mathbb{Z}}\left|\psi\left(2^{j} \sqrt{L}\right) f\right|^{2}\right)^{1 / 2} .
$$

Then $\mathcal{G}_{L}$ is bounded on $L^{p}(X)$ for all $p_{0}<p<p_{0}^{\prime}$.

Proof. The finite speed propagation property implies $L^{2}-L^{2}$ off-diagonal estimate

$$
\left\|P_{X \backslash B\left(x, 2^{j} r\right)} e^{-r^{2} L} P_{B(x, r)}\right\|_{2 \rightarrow 2} \leq C e^{-c 2^{2 j}},
$$

see $[19,55]$. It follows from $\left(\mathrm{E}_{\mathrm{p}_{0}, 2}\right)$ that

$$
\left\|P_{X \backslash B\left(x, 2^{j} r\right)} e^{-r^{2} L} P_{B(x, r)}\right\|_{p_{0} \rightarrow 2} \leq C V(x, r)^{\frac{1}{2}-\frac{1}{p_{0}}} .
$$

Now the Riesz-Thorin interpolation theorem gives for $p \in\left(p_{0}, 2\right)$ the following $L^{p}-L^{2}$ off-diagonal estimate

$$
\left\|P_{X \backslash B\left(x, 2^{j} r\right)} e^{-r^{2} L} P_{B(x, r)}\right\|_{p \rightarrow 2} \leq C V(x, r)^{\frac{1}{2}-\frac{1}{p}} e^{-c^{\prime} 2^{2 j}} .
$$

Now assertion (a) follows from [6]. The latter off-diagonal estimate implies that $L$ has a bounded holomorphic functional calculus on $L^{p}$ for $p_{0}<p<p_{0}^{\prime}$ (see [7]). It is known that the holomorphic functional calculus implies the quadratic estimate of assertion (b) (see $[20,45])$.

Throughout the rest of this section, $\Phi$ denotes an even function, $\Phi \in \mathscr{S}(\mathbb{R})$ such that $\Phi(0)=1$ and whose Fourier transform $\hat{\Phi}$ is supported in $[-1,1]$. We take $\eta \in C_{c}^{\infty}(\mathbb{R})$ even and such that $\operatorname{supp} \eta \subseteq\{\xi: 1 / 2 \leq|\xi| \leq 2\}$ and $\sum_{\ell \in \mathbb{Z}} \eta\left(2^{-\ell} \lambda\right)=1$ for all $\lambda>0$. Set $\eta_{\ell}(\lambda)=\eta\left(2^{\ell} \lambda\right)$ and

$$
\widehat{F^{(\ell)}}=\eta_{-\ell} \widehat{F}, \quad \ell \in \mathbb{Z} \text {. }
$$

Put $Q_{\ell}(\lambda)=\sum_{k \geq 0} \eta_{k+\ell}(\lambda)$.

Proposition 4.6. Suppose that operator L satisfies property $(\mathrm{FS})$ and condition $\left(\mathrm{E}_{\mathrm{p}_{0}, 2}\right)$ for $1 \leq$ $p_{0}<2$ and let $p_{0}<p<2$. Assume in addition that for an even bounded Borel function $F$ the following estimates hold

$$
\sum_{k<0} \sup _{j}\left\|\sum_{\ell \geq 0} F^{(j+\ell)}(\sqrt{L}) \eta_{j+k}(\sqrt{L})\right\|_{p \rightarrow p}<\infty
$$


and

$$
\sup _{j}\left\|\sum_{\ell \geq 0} F^{(j+\ell)}(\sqrt{L})\left(I-\Phi\left(2^{j} \sqrt{L}\right)\right) Q_{j}(\sqrt{L})\right\|_{p \rightarrow p}<\infty .
$$

Then $F(\sqrt{L})$ is of weak-type $(p, p)$.

Proof. Let $f \in L^{p}(X)$ and $\alpha>\mu(X)^{-1 / p}\|f\|_{p}$. A simple variation of the Calderón-Zygmund decomposition of $|f|^{p}$ at height $\alpha$ shows that there exist constants $C$ and $K$ such that $f=g+b=$ $g+\sum_{i} b_{i}$ so that $\|g\|_{p} \leq C\|f\|_{p},\|g\|_{\infty} \leq C \alpha$, each $b_{i}$ is supported on ball $B_{i}$ of radius $2^{j(i)}$, and $\#\left\{i: x \in 8 B_{i}\right\} \leq K$ for all $x \in X, \int_{X}\left|b_{i}\right|^{p} d \mu \leq C \alpha^{p} \mu\left(B_{i}\right)$, and $\sum_{i} \mu\left(B_{i}\right) \leq C \alpha^{-p}\|f\|_{p}^{p}$. As a consequence, $\alpha^{p-2}\|g\|_{2}^{2} \leq C\|f\|_{p}^{p}$. As in [11], we choose $2^{j(i)}$ rather than $r(i)$ to be able to sum in $j$.

We define the "nearly good" and "very bad" functions $\tilde{g}$ and $\tilde{b}$ by

$$
\tilde{g}=g+\sum_{i} 2 \Phi\left(2^{j(i)} \sqrt{L}\right) b_{i}-\sum_{i} \Phi^{2}\left(2^{j(i)} \sqrt{L}\right) b_{i} \quad \text { and } \quad \tilde{b}=\sum_{i}\left(I-\Phi\left(2^{j(i)} \sqrt{L}\right)\right)^{2} b_{i} .
$$

By Lemma 2.1, $\operatorname{supp} \Phi\left(2^{j(i)} \sqrt{L}\right) b_{i} \subset 4 B_{i}$, and by the Calderón-Zygmund decomposition, every $x \in X$ belongs to no more than $K$ balls $4 B_{i}$. Let $N>n(1 / p-1 / 2)$. Now by condition $\left(\mathrm{E}_{\mathrm{p}_{0}, 2}\right)$

$$
\begin{aligned}
\left\|\sum_{i} \Phi\left(2^{j(i)} \sqrt{L}\right) b_{i}\right\|_{2}^{2} & \leq K \sum_{i}\left\|\Phi\left(2^{j(i)} \sqrt{L}\right) b_{i}\right\|_{2}^{2} \\
& \leq C \sum_{i} \sup _{\lambda}\left|\Phi\left(2^{j(i)} \lambda\right)\left(1+2^{j(i)} \lambda\right)^{N}\right|^{2}\left\|\left(1+2^{j(i)} \sqrt{L}\right)^{-N} b_{i}\right\|_{2}^{2} \\
& \leq C \sum_{i} \mu\left(B_{i}\right)^{1-\frac{2}{p_{0}}}\left\|b_{i}\right\|_{p_{0}}^{2} \\
& \leq C \sum_{i} \mu\left(B_{i}\right)^{1-\frac{2}{p_{0}}}\left\|b_{i}\right\|_{p}^{2} \mu\left(B_{i}\right)^{\frac{2}{p_{0}}-\frac{2}{p}} \\
& \leq C \alpha^{2} \sum_{i} \mu\left(B_{i}\right) \leq C \alpha^{2-p}\|f\|_{p}^{p} .
\end{aligned}
$$

Replacing $\Phi$ by $\Phi^{2}$ yields $\left\|\sum_{i} \Phi^{2}\left(2^{j(i)} \sqrt{L}\right) b_{i}\right\|_{2}^{2} \leq C \alpha^{2-p}\|f\|_{p}^{p}$. By the standard $L^{2}$ argument

$$
\mu(\{x: F(\sqrt{L})(\tilde{g})(x)>\alpha\}) \leq C \alpha^{-p}\|f\|_{p}^{p} .
$$

It remains to treat

$$
\begin{aligned}
F(\sqrt{L})\left(\sum_{i}\left(I-\Phi\left(2^{j(i)} \sqrt{L}\right)\right)^{2} b_{i}\right) & =\sum_{i} \sum_{\ell \geq 0} F^{(j(i)+\ell)}(\sqrt{L})\left(I-\Phi\left(2^{j(i)} \sqrt{L}\right)\right)^{2} b_{i} \\
& +\sum_{i} \sum_{\ell<0} F^{(j(i)+\ell)}(\sqrt{L})\left(I-\Phi\left(2^{j(i)} \sqrt{L}\right)\right)^{2} b_{i} .
\end{aligned}
$$

By Lemma 2.1

$$
\operatorname{supp} F^{(j(i)+\ell)}(\sqrt{L})\left(I-\Phi\left(2^{j(i)} \sqrt{L}\right)\right)^{2} b_{i} \subseteq B\left(x_{i}, 2^{j(i)+\ell+1}+3 \cdot 2^{j(i)}\right) \subseteq 8 B_{i}, \quad \forall \ell<0 .
$$

Thus the second term of (4.5) is supported in $\cup 8 B_{i}$ and $\sum \mu\left(8 B_{i}\right) \leq C \sum \mu\left(B_{i}\right) \leq C \alpha^{-p}\|f\|_{p}^{p}$.

To treat the first term we show that

$$
\left\|\sum_{j} \sum_{\ell \geq 0} F^{(j+\ell)}(\sqrt{L})\left(I-\Phi\left(2^{j} \sqrt{L}\right)\right)^{2} f_{j}\right\|_{p} \leq C\left\|\sum_{j}\left|f_{j}\right|\right\|_{p} .
$$

If we apply (4.6) with

$$
f_{j}=\sum_{i: j(i)=j} b_{i}
$$


we see that

$$
\begin{aligned}
\left\|\sum_{i} \sum_{\ell \geq 0} F^{(j(i)+\ell)}(\sqrt{L})\left(I-\Phi\left(2^{j(i)} \sqrt{L}\right)\right)^{2} b_{i}\right\|_{p} & =\left\|\sum_{j} \sum_{\ell \geq 0} F^{(j+\ell)}(\sqrt{L})\left(I-\Phi\left(2^{j} \sqrt{L}\right)\right)^{2} f_{j}\right\|_{p} \\
& \leq C\left\|\sum_{j} \mid \sum_{i: j(i)=j} b_{i}\right\|_{p} \\
& \leq C\left\|\sum_{i}\left|b_{i}\right|\right\|_{p} \\
& \leq C\left(\sum_{i}\left\|b_{i}\right\|_{p}^{p}\right)^{1 / p} \leq C\|f\|_{p},
\end{aligned}
$$

which completes the proof.

As in [11] we argue that by duality, (4.6) is equivalent to

$$
\left\|\sup _{j}\left|\sum_{\ell \geq 0} \bar{F}^{(j+\ell)}(\sqrt{L})\left(I-\Phi\left(2^{j} \sqrt{L}\right)\right)^{2} h\right|\right\|_{p^{\prime}} \leq C\|h\|_{p^{\prime}} .
$$

Write

$$
\begin{aligned}
\sum_{\ell \geq 0} \bar{F}^{(j+\ell)}(\sqrt{L})\left(I-\Phi\left(2^{j} \sqrt{L}\right)\right)^{2} & =\sum_{k<0} \sum_{\ell \geq 0} \bar{F}^{(j+\ell)}(\sqrt{L})\left(I-\Phi\left(2^{j} \sqrt{L}\right)\right)^{2} \eta_{j+k}(\sqrt{L}) \\
& +\sum_{\ell \geq 0} \bar{F}^{(j+\ell)}(\sqrt{L})\left(I-\Phi\left(2^{j} \sqrt{L}\right)\right)^{2} Q_{j}(\sqrt{L}) .
\end{aligned}
$$

Let $\tilde{\eta} \in C_{c}^{\infty}(0, \infty)$ be a non-negative function satisfying supp $\tilde{\eta} \subseteq[1 / 4,4]$ and $\tilde{\eta}=1$ on $[1 / 2,2]$, and let $\tilde{\eta}_{j}$ denote the function $\tilde{\eta}\left(2^{j} \cdot\right)$. By Lemma 4.5 point (b) with $\psi=\tilde{\eta}$ there exists a constant $C>0$ independent of $k<0$ such that for $2<p^{\prime}<p_{0}^{\prime}$,

$$
\left(\sum_{j}\left\|\tilde{\eta}_{j+k}(\sqrt{L}) h\right\|_{p^{\prime}}^{p^{\prime}}\right)^{1 / p^{\prime}} \leq\left\|\left(\sum_{j}\left|\tilde{\eta}_{j+k}(\sqrt{L}) h\right|^{2}\right)^{1 / 2}\right\|_{p^{\prime}} \leq C\|h\|_{p^{\prime}},
$$

and by Lemma 4.5 point (a), it follows that for $2<p^{\prime}<p_{0}^{\prime},\left\|\left(I-\Phi\left(2^{j} \sqrt{L}\right)\right)^{2}\right\|_{p^{\prime} \rightarrow p^{\prime}} \leq C$ for some constant $C>0$ independent of $j \in \mathbb{Z}$. Hence

$$
\begin{aligned}
& \| \sup _{j} \mid \sum_{k<0} \sum_{\ell \geq 0} \bar{F}^{(j+\ell)}(\sqrt{L})\left(I-\Phi\left(2^{j} \sqrt{L}\right)\right)^{2} \eta_{j+k}(\sqrt{L}) h\|\|_{p^{\prime}} \\
& \leq \sum_{k<0}\left(\sum_{j}\left\|\sum_{\ell \geq 0} \bar{F}^{(j+\ell)}(\sqrt{L})\left(I-\Phi\left(2^{j} \sqrt{L}\right)\right)^{2} \eta_{j+k}(\sqrt{L}) \tilde{\eta}_{j+k}(\sqrt{L}) h\right\|_{p^{\prime}}^{p^{\prime}}\right)^{1 / p^{\prime}} \\
& \leq C \sum_{k<0} \sup _{j}\left\{\left\|\left(I-\Phi\left(2^{j} \sqrt{L}\right)\right)^{2}\right\|_{p^{\prime} \rightarrow p^{\prime}}\left\|\sum_{\ell \geq 0} \bar{F}^{(j+\ell)}(\sqrt{L}) \eta_{j+k}(\sqrt{L})\right\|_{p^{\prime} \rightarrow p^{\prime}}\right\} \\
& \quad \times\left(\sum_{j}\left\|\tilde{\eta}_{j+k}(\sqrt{L}) h\right\|_{p^{\prime}}^{p^{\prime}}\right)^{1 / p^{\prime}} \\
& \leq C \sum_{k<0} \sup _{j}\left\|\sum_{\ell \geq 0} \bar{F}^{(j+\ell)}(\sqrt{L}) \eta_{j+k}(\sqrt{L})\right\|_{p^{\prime} \rightarrow p^{\prime}}\|h\|_{p^{\prime}} \leq C\|h\|_{p^{\prime}} .
\end{aligned}
$$

The last inequality follows from assumption (4.3). Using assumption (4.4) instead of (4.3) the similar argument as above gives the following estimate

$$
\left\|\sup _{j} \mid \sum_{\ell \geq 0} \bar{F}^{(j+\ell)}(\sqrt{L})\left(I-\Phi\left(2^{j} \sqrt{L}\right)\right)^{2} Q_{j}(\sqrt{L}) h\right\|\left\|_{p^{\prime}} \leq C\right\| h \|_{p^{\prime}} .
$$

This shows (4.7) and ends the proof of Proposition 4.6. 
Proposition 4.7. Suppose that operator L satisfies property (FS) and condition $\left(\mathrm{E}_{\mathrm{p}_{0}, 2}\right)$ for some $1 \leq p_{0}<2$ and fix $p \in\left(p_{0}, 2\right)$. Next assume that for all even Borel functions $H$ such that $\operatorname{supp} H$ is compact and $\|H\|_{W^{\beta, \infty}}<\infty$ for some $\beta>n / 2$,

$$
\sup _{t>0}\|H(t \sqrt{L})\|_{p \rightarrow p} \leq C\|H\|_{W^{\beta, \infty}} .
$$

Then for any even bounded Borel function $F$ such that $\left\|\left(F \eta_{i}\right)^{(j)}(\sqrt{L})\right\|_{p \rightarrow p} \leq \alpha(i-j)$ for all $i, j \in \mathbb{Z}$ with

$$
\sum_{k \leq 0}(|k|+1) \alpha(k)<\infty
$$

the operator $F(\sqrt{L})$ is of weak-type $(p, p)$.

Proof. By Proposition 4.6, it suffices to verify (4.3) and (4.4). Note that we may assume $F(0)=0$ since $F=F-F(0)+F(0)$.

Firstly we show (4.3). Fix $k \leq 0, \ell \geq 0$ and $j \in \mathbb{Z}$ and write

$$
\begin{aligned}
F^{(j+\ell)}(\sqrt{L}) \eta_{j+k}(\sqrt{L}) & =\sum_{i \in \mathbb{Z}}\left(F \eta_{i+j}\right)^{(j+\ell)}(\sqrt{L}) \eta_{j+k}(\sqrt{L}) \\
& =\sum_{i<k-2}+\sum_{i=k-2}^{k+2}+\sum_{i>k+2} \cdots=I_{j k \ell}+I_{j k \ell}+I I I_{j k \ell} .
\end{aligned}
$$

The main term is $I I_{j k \ell} ; I_{j k \ell}$ and $I I I_{j k \ell}$ are error terms. By (4.10), $\left\|\eta_{j+k}(\sqrt{L})\right\|_{p \rightarrow p} \leq C$ so

$$
\left\|I_{j k \ell}\right\|_{p \rightarrow p} \leq C \sum_{i=k-1}^{k+1}\left\|\left(F \eta_{i+j}\right)^{(j+\ell)}(\sqrt{L})\right\|_{p \rightarrow p} \leq C \sum_{i=-2}^{2} \alpha(k+i-\ell) .
$$

Thus,

$$
\sum_{k \leq 0} \sum_{\ell \geq 0} \sup _{j}\left\|\boldsymbol{I}_{j k \ell}\right\|_{p \rightarrow p} \leq C \sum_{m \leq 2}(|m|+1) \alpha(m)<\infty .
$$

To estimate $\left\|I_{j k \ell}\right\|_{p \rightarrow p}$ set

$$
G(\lambda)=\sum_{i<k-2}\left(F \eta_{i+j}\right)^{(j+\ell)}\left(2^{-j-k} \lambda\right) \eta(\lambda)
$$

Observe that

$$
\frac{d^{\gamma}}{d \lambda^{\gamma}}\left(\sum_{i<k-2}\left(F \eta_{i+j}\right)^{(j+\ell)}\left(2^{-j-k} \lambda\right)\right)=\int_{|s| \geq 2^{-k-j+2}} \sum_{i<k-2} F(s) \eta_{i+j}(s) 2^{j+\ell} 2^{(\ell-k) \gamma}(\check{\eta})^{(\gamma)}\left(2^{\ell-k} \lambda-2^{j+\ell} s\right) d s .
$$

Now $2^{j+\ell}|s| \geq 2^{\ell-k+2} \geq 2^{\ell-k+1}|\lambda|$ for $\lambda \in[1 / 2,2]$. We may estimate the integral for each $N \in \mathbb{N}$ by

$$
\int_{|s| \geq 2^{-k-j+2}}\|F\|_{\infty} 2^{j+\ell} 2^{(\ell-k) \gamma} \frac{C_{N}}{\left(2^{j+\ell}|s|\right)^{N}} d s .
$$

If $N$ is chosen sufficiently large, this is dominated by $\|F\|_{\infty} 2^{(\ell-k)(1-N+\gamma)}$. This yields

$$
\|G\|_{W^{\gamma, \infty}} \leq C\|F\|_{\infty} 2^{\varepsilon_{0}(k-\ell)}
$$

for some $\varepsilon_{0}>0$ and all $\gamma \in \mathbb{N}$. Then by (4.10) $\left\|I_{j k \ell}\right\|_{p \rightarrow p} \leq C\|F\|_{\infty} 2^{\varepsilon_{0}(k-\ell)}$. Hence

$$
\sum_{k \leq 0} \sum_{\ell \geq 0} \sup _{j}\left\|I_{j k \ell}\right\|_{p \rightarrow p}<\infty .
$$

We estimat $I I I_{j k \ell}$ in the similar way as $\left\|I_{j k \ell}\right\|_{p \rightarrow p}$. This proves (4.3). 
Secondly we show (4.4). Fix $\ell \geq 0$ and $j \in \mathbb{Z}$. Write

$$
\begin{aligned}
F^{(j+\ell)}(\sqrt{L})\left(I-\Phi\left(2^{j} \sqrt{L}\right)\right) Q_{j}(\sqrt{L}) & =\sum_{i \in \mathbb{Z}}\left(F \eta_{i+j}\right)^{(j+\ell)}(\sqrt{L})\left(I-\Phi\left(2^{j} \sqrt{L}\right)\right) Q_{j}(\sqrt{L}) \\
& =\sum_{i \leq 0}+\sum_{i>0} \cdots=A_{j \ell}+B_{j \ell} .
\end{aligned}
$$

We start with the term $A_{j \ell}$. By condition (4.10) there exists a positive constant $C$ independent of $j$ such that $\left\|\left(I-\Phi\left(2^{j} \sqrt{L}\right)\right) Q_{j}(\sqrt{L})\right\|_{p \rightarrow p} \leq C$. By assumptions of the proposition, we have $\left\|\left(F \eta_{i+j}\right)^{(j+\ell)}(\sqrt{L})\right\|_{p \rightarrow p} \leq \alpha(i-l)$. Hence

$$
\sup _{j}\left\|\sum_{\ell \geq 0} A_{j \ell}\right\|_{p \rightarrow p} \leq C \sum_{\ell \geq 0} \sum_{i \leq 0} \alpha(i-\ell) \leq C \sum_{m \leq 0}(|m|+1) \alpha(m)<\infty .
$$

It remains to treat the term $B_{j \ell}$. More precisely, we want to prove that

$$
\begin{aligned}
& \sup _{j} \sum_{\ell \geq 0}\left\|\sum_{i>0}\left(F \eta_{i+j}\right)^{(j+\ell)}(\sqrt{L})\left(I-\Phi\left(2^{j} \sqrt{L}\right)\right) Q_{j}(\sqrt{L})\right\|_{p \rightarrow p} \\
& =\sup _{j} \sum_{\ell \geq 0}\left\|\sum_{i>0} \sum_{k \in \mathbb{Z}}\left(F \eta_{i+j}\right)^{(j+\ell)}(\sqrt{L}) \eta_{j+k}(\sqrt{L})\left(I-\Phi\left(2^{j} \sqrt{L}\right)\right) Q_{j}(\sqrt{L})\right\|_{p \rightarrow p}<\infty .
\end{aligned}
$$

We write

as

$$
\sum_{\mathrm{p} 0} \sum_{\mathrm{k}}
$$

$$
\begin{aligned}
\sum_{k \leq 0} \sum_{i=1}^{\infty}+\sum_{k=1}^{\ell} \sum_{i=1}^{k}+\sum_{k=1}^{\ell} \sum_{i=k+1}^{\ell}+\sum_{k=1}^{\ell} \sum_{i=\ell+1}^{\infty}+\sum_{k=\ell+1}^{\infty} \sum_{\substack{i=1 \\
=I_{j \ell}+I I_{j \ell}+I I I_{j \ell}+I_{j \ell}+V_{j \ell}+V_{j \ell}}}^{\infty} \sum_{i=\ell+1}^{\infty} \ldots
\end{aligned}
$$

Claim 1. For $k \leq 0$,

$$
\left\|\sum_{i>0}\left(F \eta_{i+j}\right)^{(j+\ell)}(\sqrt{L}) \eta_{j+k}(\sqrt{L})\right\|_{p \rightarrow p} \leq C 2^{-\varepsilon(\ell-k)}
$$

for some $\varepsilon>0$.

Claim 2.

$$
\sum_{i=1}^{k}\left\|\left(F \eta_{i+j}\right)^{(j+\ell)}(\sqrt{L})\right\|_{p \rightarrow p} \leq \sum_{i=1}^{k} \alpha(i-\ell) .
$$

Claim 3. For $k \leq i,\left\|\left(F \eta_{i+j}\right)^{(j+\ell)}(\sqrt{L}) \eta_{j+k}(\sqrt{L})\right\|_{p \rightarrow p} \leq C 2^{-\varepsilon(\ell-k)}$, so that

$$
\sum_{i=k+1}^{\ell}\left\|\left(F \eta_{i+j}\right)^{(j+\ell)}(\sqrt{L}) \eta_{j+k}(\sqrt{L})\right\|_{p \rightarrow p} \leq C(\ell-k) 2^{-\varepsilon(\ell-k)} \leq C 2^{-\varepsilon^{\prime}(\ell-k)} .
$$

Claim 4. For $\ell \geq k$,

$$
\left\|\sum_{i=\ell+1}^{\infty}\left(F \eta_{i+j}\right)^{(j+\ell)}(\sqrt{L}) \eta_{j+k}(\sqrt{L})\right\|_{p \rightarrow p} \leq C 2^{-\varepsilon^{\prime}(\ell-k)}
$$

Claim 5.

$$
\sum_{i=1}^{\ell}\left\|\left(F \eta_{i+j}\right)^{(j+\ell)}(\sqrt{L})\right\|_{p \rightarrow p} \leq \sum_{i=1}^{\ell} \alpha(i-\ell)
$$


Claim 6. For $k \geq \ell$,

$$
\left\|\sum_{i=\ell+1}^{\infty}\left(F \eta_{i+j}\right)^{(j+\ell)}(\sqrt{L}) \eta_{j+k}(\sqrt{L})\right\|_{p \rightarrow p} \leq C .
$$

If we agree with the claims 1-6, we can finish the proof of the proposition as follows. Let $\tilde{\eta} \in$ $C_{c}^{\infty}(0, \infty)$ be a non-negative function as in (4.9). We note that by condition (4.10),

$$
\begin{aligned}
\left\|\tilde{\eta}_{j+k}(\sqrt{L})\left(I-\Phi\left(2^{j} \sqrt{L}\right)\right) Q_{j}(\sqrt{L})\right\|_{p \rightarrow p} & \leq C\left\|\tilde{\eta}\left(1-\delta_{2^{-k}} \Phi\right)\right\|_{W^{n, \infty}} \\
& \leq C \min \left\{1,2^{-k}\right\} .
\end{aligned}
$$

By Claim 1,

by Claim 2 and (4.13),

$$
\sum_{\ell \geq 0}\left\|I_{j \ell}\right\|_{p \rightarrow p} \leq C \sum_{\ell \geq 0} \sum_{k \leq 0} 2^{-\varepsilon(\ell-k)}<\infty
$$

$$
\left\|\boldsymbol{I}_{j \ell}\right\|_{p \rightarrow p} \leq C \sum_{k=1}^{\ell} \sum_{i=1}^{k} \alpha(i-\ell) 2^{-\varepsilon^{\prime \prime} k}
$$

by Claim 3 and (4.13),

$$
\|I I I\|_{j \ell} \|_{p \rightarrow p} \leq C \sum_{k=1}^{\ell} 2^{-\varepsilon^{\prime}(\ell-k)} 2^{-\varepsilon^{\prime \prime} k} \leq C 2^{-\varepsilon \ell}
$$

by Claim 4 and (4.13),

$$
\left\|N_{j \ell}\right\|_{p \rightarrow p} \leq C \sum_{k=1}^{\ell} 2^{-\varepsilon^{\prime}(\ell-k)} 2^{-\varepsilon^{\prime \prime} k} \leq C 2^{-\varepsilon \ell}
$$

by Claim 5 and (4.13),

$$
\left\|V_{j \ell}\right\|_{p \rightarrow p} \leq C \sum_{k=\ell+1}^{\infty} \sum_{i=1}^{\ell} \alpha(i-\ell) 2^{-\varepsilon^{\prime \prime} k}
$$

by Claim 6 and (4.13),

$$
\left\|V I_{j \ell}\right\|_{p \rightarrow p} \leq C \sum_{k=\ell+1}^{\infty} 2^{-\varepsilon^{\prime \prime} k} \leq 2^{-\varepsilon \ell} .
$$

Hence

$$
\sum_{\ell>0}\left\|I I I_{j \ell}+N_{j \ell}+V I_{j \ell}\right\|_{p \rightarrow p} \leq C \sum_{\ell>0} 2^{-\varepsilon \ell}<\infty
$$

and

$$
\begin{aligned}
\left\|I_{j \ell}+V_{j \ell}\right\|_{p \rightarrow p} & \leq C \sum_{k=1}^{\ell} \sum_{i=1}^{k} \alpha(i-\ell) 2^{-\varepsilon^{\prime \prime} k}+C \sum_{k=\ell+1}^{\infty} \sum_{i=1}^{\ell} \alpha(i-\ell) 2^{-\varepsilon^{\prime \prime} k} \\
& =C \sum_{i=1}^{\ell} \alpha(i-\ell) \sum_{k=i}^{\infty} 2^{-\varepsilon k} \leq C \sum_{i=1}^{\ell} \alpha(i-\ell) 2^{-\varepsilon i} .
\end{aligned}
$$

Thus

$$
\begin{aligned}
\sum_{\ell>0}\left\|I_{j \ell}+V_{j \ell}\right\|_{p \rightarrow p} & \leq C \sum_{\ell>0} \sum_{i=1}^{\ell} 2^{-\varepsilon i} \alpha(i-\ell) \\
& \leq C \sum_{m \leq 0} \alpha(m) 2^{-\varepsilon m} \sum_{\ell \geq-m} 2^{-\varepsilon \ell} \leq C \sum_{m \leq 0} \alpha(m)<\infty
\end{aligned}
$$


Now Claims 2 and 5 follow immediately from the definition of $\alpha(j)$. Similarly to the proof of (4.12), we use condition (4.10) to prove Claims 1, 3, 4 and 6. So to establish Claims 1, 3, 4 and 6 we examine

$$
\frac{d^{\gamma}}{d \lambda^{\gamma}}\left(\sum_{i}\left(F \eta_{i+j}\right)^{(j+\ell)}\left(2^{-j-k} \lambda\right)\right)
$$

for $|\lambda| \sim 1$, (where the sum is over a range of $i$ depending on which claim we are proving). In any case, we obtain

$$
\int \sum_{i} F(s) \eta_{i+j}(s) 2^{j+\ell} 2^{(\ell-k) \gamma} \frac{d^{\gamma} \check{\eta}}{d \lambda^{\gamma}}\left(2^{\ell-k} \lambda-2^{j+\ell} s\right) d s .
$$

For Claim 6, we just use $\left|\frac{d^{\gamma} \check{\eta}}{d \lambda^{\gamma}}\right| \leq C$ and the fact that the integrand is supported in a set of measure $\leq C 2^{-(j+\ell)}$ to estimate the integral by $\|F\|_{\infty} 2^{(\ell-k) \gamma}$; under the hypotheses of Claims 1,3 and 4 we have that (essentially) $2^{\ell-k}|\lambda| \geq 2 \cdot 2^{j+\ell}|s|$ if $|\lambda| \sim 1$, and thus we may estimate the integral by $\|F\|_{\infty} 2^{j+\ell} 2^{(\ell-k)(\gamma-N)}$ multiplied by the measure of the support of the integrand, for each $N \in \mathbb{N}$. These measures are $2^{-j}, 2^{-i-j}$ and $2^{-j-\ell}$ respectively, and so in each case we can dominate the integral by $C 2^{(\ell-k)(\gamma-N+1)}$ for all $N \in \mathbb{N}$.

Recall that for $0<\alpha<1, \Lambda_{\alpha}$ is the usual Lipschitz space as defined for example in [60]. As a consequence of Proposition 4.7, we have the following result.

Corollary 4.8. Assume that operator $L$ satisfies property (FS) and condition $\left(\mathrm{E}_{\mathrm{p}_{0}, 2}\right)$ for some $1 \leq p_{0}<2$. Next assume that for all even bounded Borel function $F$ such that $\operatorname{supp} F$ is compact and $\|F\|_{W^{\beta, \infty}}<\infty$ for some $\beta>n / 2$,

$$
\sup _{t>0}\|F(t \sqrt{L})\|_{p \rightarrow p} \leq C\|F\|_{W^{\beta, \infty}}, \quad p_{0}<p<p_{0}^{\prime} .
$$

Then for any bounded Borel function $F$ such that for all $i, j \in \mathbb{Z}$,

$$
\left\|\left(F \eta_{i}\right)^{(j)}(\sqrt{L})\right\|_{r \rightarrow r}+\left\|\eta \delta_{2^{-i}} F\right\|_{\Lambda_{\alpha}} \leq C
$$

for some $0<\alpha<1$ and all $p_{0}<r \leq 2, F(\sqrt{L})$ is bounded on $L^{r}$ for $p_{0}<r<p_{0}^{\prime}$.

Proof. First, we note that $\left\|\eta \delta_{2^{-i}} F\right\|_{\Lambda_{\alpha}} \leq C$ is equivalent to $\left\|\left(F \eta_{i}\right)^{(j)}(\sqrt{L})\right\|_{2 \rightarrow 2} \leq 2^{\alpha(i-j)}$. By interpolation, there exists some $\alpha^{\prime}>0$ such that

$$
\left\|\left(F \eta_{i}\right)^{(j)}(\sqrt{L})\right\|_{r \rightarrow r} \leq C 2^{\alpha^{\prime}(i-j)}
$$

for all $p_{0}<r \leq 2$. From Proposition 4.7, $F(\sqrt{L})$ is of weak-type $(r, r)$ for all $p_{0}<r \leq 2$. By duality and interpolation, $F(\sqrt{L})$ is bounded on $L^{r}$ for $p_{0}<r<p_{0}^{\prime}$.

Proof of Theorem 4.4. It is enough to verify conditions (4.15) of Corollary 4.8. First we note that by definition

$$
\left(F \eta_{i}\right)^{(j)}(\lambda)=\int F\left(2^{-i} t\right) \eta(t) \widehat{\eta_{i-j}}\left(2^{i} \lambda-t\right) d t=\left(\delta_{2^{-i}} F \eta\right)^{(j-i)}\left(2^{i} \lambda\right),
$$

so $\left\|\left(F \eta_{i}\right)^{(j)}(\sqrt{L})\right\|_{r \rightarrow r}=\left\|\left(\delta_{2^{-i}} F \eta\right)^{(j-i)}\left(2^{i} \sqrt{L}\right)\right\|_{r \rightarrow r}$. Let $\psi$ be a $C_{c}^{\infty}$ even function which is supported on $[-8,8]$ and $\psi(\lambda)=1$ on $[-4,4]$. Write

$$
\left\|\left(\delta_{2^{-i}} F \eta\right)^{(j-i)}\left(2^{i} \sqrt{L}\right)\right\|_{r \rightarrow r} \leq\left\|\left(\psi\left(\delta_{2^{-i}} F \eta\right)^{(j-i)}\right)\left(2^{i} \sqrt{L}\right)\right\|_{r \rightarrow r}+\left\|\left((1-\psi)\left(\delta_{2^{-i}} F \eta\right)^{(j-i)}\right)\left(2^{i} \sqrt{L}\right)\right\|_{r \rightarrow r} .
$$

Observe that the function $\psi\left(\delta_{2^{-i}} F \eta\right)^{(j-i)}$ is supported on $[-8,8]$ and

$$
\begin{aligned}
\left\|\psi\left(\delta_{2^{-i}} F \eta\right)^{(j-i)}\right\|_{W^{\beta, q}} & \leq C\left\|\left(\delta_{2^{-i}} F \eta\right)^{(j-i)}\right\|_{W^{\beta, q}} \\
& =C\left\|\mathscr{F}^{-1}\left(\left(1+\xi^{2}\right)^{\beta / 2} \widehat{\delta_{2^{-i}} F} \eta(\xi) \eta_{i-j}(\xi)\right)\right\|_{q} \\
& \leq C\left\|\mathscr{F}^{-1}\left(\left(1+\xi^{2}\right)^{\beta / 2} \widehat{\delta_{2^{-i}} F} \eta(\xi)\right)\right\|_{q}
\end{aligned}
$$




$$
=C\left\|F\left(2^{-i} \lambda\right) \eta(\lambda)\right\|_{W^{\beta, q}} .
$$

From condition (4.2), we have that $\left\|\eta(\lambda) F\left(2^{-i} \lambda\right)\right\|_{W^{\beta, q}}<\infty$. Therefore, we use our assumption (4.1) to obtain

$$
\left\|\left(\psi\left(\delta_{2^{-i}} F \eta\right)^{(j-i)}\right)\left(2^{i} \sqrt{L}\right)\right\|_{r \rightarrow r} \leq C
$$

with $C>0$ independent of $i$ and $j$.

We estimate the term $\left\|\left((1-\psi)\left(\delta_{2^{-i}} F \eta\right)^{(j-i)}\right)\left(2^{i} \sqrt{L}\right)\right\|_{r \rightarrow r}$. For $k \in \mathbb{N}$ and all $\lambda \neq 0$, we have by elementary calculation,

$$
\begin{aligned}
& \left.\mid(1-\psi(\lambda)) \frac{d^{k}}{d \lambda^{k}}\left(\left(\delta_{2^{-i}} F \eta\right)^{(j-i)}\right)(\lambda)\right) \mid \\
& \quad \leq C 2^{(j-i)(k+1)}|1-\psi(\lambda)| \int\left|F\left(2^{-i} u\right) \eta(u)\right|\left(1+2^{j-i}|\lambda-u|\right)^{-k-1} d u \leq C|\lambda|^{-k},
\end{aligned}
$$

where we use the fact that $|\lambda| \geq 4$ and $|u| \leq 2$. We then apply (a) of Lemma 4.5 to obtain

$$
\left\|\left((1-\psi)\left(\delta_{2^{-i}} F \eta\right)^{(j-i)}\right)\left(2^{i} \sqrt{L}\right)\right\|_{r \rightarrow r} \leq C .
$$

This estimate in combination with (4.16) shows that

$$
\left\|\left(F \eta_{i}\right)^{(j)}(\sqrt{L})\right\|_{r \rightarrow r}=\left\|\left(\delta_{2^{-i}} F \eta\right)^{(j-i)}\left(2^{i} \sqrt{L}\right)\right\|_{r \rightarrow r} \leq C
$$

for some constant $C>0$ independent of $i, j$.

Now we recall that if $1 \leq q \leq \infty$ and $\beta-1 / q>0$, then

$$
W^{\beta, q} \subseteq B_{q, \infty}^{\beta} \subseteq B_{\infty, \infty}^{\beta-\frac{1}{q}} \subseteq \Lambda_{\min \left\{\beta-\frac{1}{q}, \frac{1}{2}\right\}}
$$

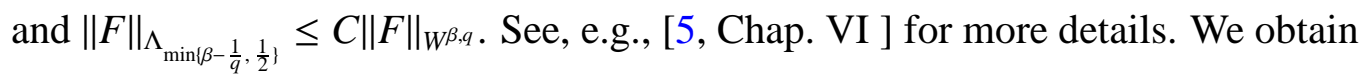

$$
\left\|\eta \delta_{2^{-i}} F\right\|_{\Lambda_{\min \{\beta-1 / q, 1 / 2\}}} \leq C\left\|\eta \delta_{2^{-i}} F\right\|_{W^{\beta, q}} \leq C^{\prime} .
$$

This estimate and (4.17) prove condition (4.15) of Corollary 4.8. The proof of Theorem 4.4 is finished.

\section{ENDPOINT ESTIMATES FOR BOCHNER-RIESZ MEANS}

We have seen in Corollary 3.2 that Bochner-Riesz means are bounded on $L^{p}$ provided the order $\delta$ satisfies $\delta>\max \{n(1 / p-1 / s)-1 / q, 0\}$. In this section we prove that our restriction type condition implies endpoint estimates for Bochner-Riesz means. Our approach is inspired by the results of Christ and Tao $[14,15,63]$. As in the rest of the paper we assume that $(X, d, \mu)$ is a metric measure space satisfying condition (2.2) with a homogeneous dimension $n$.

For any given $p \in[1,2)$ and $q \in[1, \infty]$ we define

$$
\delta_{q}(p)=\max \left\{0, n\left|\frac{1}{p}-\frac{1}{2}\right|-\frac{1}{q}\right\} .
$$

For simplicity we will write $\delta(p)$ instead of $\delta_{2}(p)$. As in Sections 3 and 4 we discuss two type of results corresponding to estimates $\left(\mathrm{ST}_{\mathrm{p}, 2}^{\mathrm{q}}\right)$ or $\left(\mathrm{SC}_{\mathrm{p}, 2}^{\mathrm{q}, 1}\right)$.

Theorem 5.1. Assume that operator $L$ satisfies property $(\mathrm{FS})$ and condition $\left(\mathrm{ST}_{\mathrm{p}, 2}^{\mathrm{q}}\right)$ for some $p, q$ satisfying $1 \leq p<2$ and $1 \leq q \leq \infty$. Then the operator $S_{R}^{\delta_{q}(p)}(L)$ is of weak-type $(p, p)$ uniformly in $R$. 
The next theorem is a variation of Theorem 5.1. As in Theorems 3.6 and 4.2, this variation can be used in the case of operators with nonempty pointwise spectrum.

Theorem 5.2. Assume that $\mu(X)<\infty$. Assume that operator L satisfies property (FS) and condition $\left(\mathrm{SC}_{\mathrm{p}, 2}^{\mathrm{q}, 1}\right)$ for some $p, q$ satisfying $1 \leq p<2$ and $1 \leq q \leq \infty$. Then the operator $S_{R}^{\delta_{q}(p)}(L)$ is of weak-type $(p, p)$ uniformly in $R$.

The proofs of Theorem 5.1 and 5.2 require three technical lemmas which we discuss first. We commence with the following observation.

Lemma 5.3. For each $k \leq 0$ there exists a decomposition

$$
S_{R}^{\delta_{q}(p)}\left(\lambda^{2}\right)=\eta_{k}(\lambda) n_{k}(\lambda)+S_{R}^{\delta_{q}(p)}\left(\lambda^{2}\right) n_{k}(\lambda)
$$

such that

(a) Functions $n_{k}$ are even and their Fourier transforms are supported in $\left[-2^{k} / R, 2^{k} / R\right]$, i.e., $\operatorname{supp} \widehat{n_{k}} \subset\left[-2^{k} / R, 2^{k} / R\right]$;

(b) Functions $\eta_{k}$ are continuous, even and $\sum_{k=-\infty}^{0}\left|\eta_{k}(\lambda)\right|^{2} \leq C$ with $C$ independent of $\lambda$ and $R$;

(c) For certain arbitrarily large $N \in \mathbb{N}$ there exists a constant $C$ such that

$$
\left|n_{k}(\lambda)\right| \leq C\left(1+\frac{2^{k}|\lambda|}{R}\right)^{-N}
$$

Proof. Following [63] we consider the function $\Phi(\lambda)=6 \lambda^{-3}(\lambda-\sin \lambda)$. Note that $\widehat{\Phi}(t)=3 \pi(1-$ $|t|)_{+}^{2}$. Set $n_{k}(\lambda)=\Phi^{N / 2}\left(2^{k} \lambda(R N)^{-1}\right)$. We use term $(R N)^{-1}$ rather than $R^{-1}$ to ensure that supp $\widehat{n_{k}} \subset$ $\left[-2^{k} / R, 2^{k} / R\right]$. Next we write

$$
\begin{aligned}
S_{R}^{\delta_{q}(p)}\left(\lambda^{2}\right) & =\left(\frac{S_{R}^{\delta_{q}(p)}\left(\lambda^{2}\right)\left(1-n_{k}(\lambda)\right)}{n_{k}(\lambda)}\right) n_{k}(\lambda)+S_{R}^{\delta_{q}(p)}\left(\lambda^{2}\right) n_{k}(\lambda) \\
& =\eta_{k}(\lambda) n_{k}(\lambda)+S_{R}^{\delta_{q}(p)}\left(\lambda^{2}\right) n_{k}(\lambda) .
\end{aligned}
$$

Verifying conditions (a) and (c) is straightforward. By definition of $n_{k}(\lambda)$, there exists some constant $C$ depending only on $N$ such that $n_{k}(\lambda) \geq C$ and $\left|S_{R}^{\delta_{q}(p)}\left(\lambda^{2}\right)\left(1-n_{k}(\lambda)\right)\right| \leq C 2^{k}$ for all $|\lambda| \leq R$ and $k \leq 0$. This proves condition (b) because $\operatorname{supp} S_{R}^{\delta_{q}(p)}\left(\lambda^{2}\right) \subset[-R, R]$.

The following lemma comes from [63].

Lemma 5.4. For each $k>0$, there exists a decomposition $S_{R}^{\delta_{q}(p)}\left(\lambda^{2}\right)=m_{k}(\lambda)+\eta_{k}(\lambda) n_{k}(\lambda)$ such that:

(a) Functions $\widehat{m_{k}}$ and $\widehat{n_{k}}$ are even and supported on $\left[-2^{k} / R, 2^{k} / R\right]$;

(b) $\eta_{k}$ are continuous and for all $\lambda>0, \sum_{k=1}^{\infty}\left|\eta_{k}(\lambda)\right|^{2} \leq C$ with $C$ independent of $\lambda$ and $R$;

(c) For certain arbitrarily large $N \in \mathbb{N}$ there exists a constant $C$ such that

$$
\left|n_{k}(\lambda)\right| \leq C 2^{-\delta_{q}(p) k}\left(1+2^{k}\left|1-\frac{|\lambda|}{R}\right|\right)^{-N}
$$

Proof. For the proof, we refer to Lemma 2.1 in [63].

Next we discuss the proof of the last lemma required in the proofs of Theorems 5.1 and 5.2. 
Lemma 5.5. Suppose that $L$ is a self-adjoint operator on $L^{2}(X)$. Assume that $\left\{Q_{k}\right\}_{k \in \mathbb{N}}$ is a family of continuous real-valued functions such that $\sum_{k}\left|Q_{k}(\lambda)\right|^{2} \leq C$ for some constant $C$ independent of $\lambda$. Then for any sequence of functions $\left\{f_{k}\right\}_{k \in \mathbb{N}}$ on $X$,

$$
\left\|\sum_{k} Q_{k}(\sqrt{L}) f_{k}\right\|_{2}^{2} \leq C \sum_{k}\left\|f_{k}\right\|_{2}^{2}
$$

Proof. Note that

$$
\begin{aligned}
\left\|\sum_{k} Q_{k}(\sqrt{L}) f_{k}\right\|_{2}^{2} & =\left\langle\sum_{k} Q_{k}(\sqrt{L}) f_{k}, \sum_{k} Q_{k}(\sqrt{L}) f_{k}\right\rangle \\
& =\int_{-\infty}^{+\infty} \sum_{k} \sum_{j} Q_{k}(\lambda) Q_{j}(\lambda) d\left\langle E_{\sqrt{L}}(\lambda) f_{k}, f_{j}\right\rangle .
\end{aligned}
$$

Under the assumption of Lemma 5.5 the above integral is a limit of its Riemannian approximations, see page 310 of [68]. Therefore let us consider Riemannian partition of $[\alpha, \beta]$

$$
\alpha=\lambda_{1}<\lambda_{2}<\ldots<\lambda_{n}=\beta, \quad \lambda_{l}^{\prime} \in\left(\lambda_{\ell}, \lambda_{\ell+1}\right]
$$

for some $-\infty<\alpha<\beta<\infty$. Now to finish the proof of Lemma 5.5 it is enough to observe that

$$
\begin{aligned}
& \left|\sum_{\ell} \sum_{k} \sum_{j} Q_{k}\left(\lambda_{\ell}^{\prime}\right) Q_{j}\left(\lambda_{\ell}^{\prime}\right)\left\langle E_{\sqrt{L}}\left(\lambda_{\ell}, \lambda_{\ell+1}\right] f_{k}, f_{j}\right\rangle\right| \\
& \leq \sum_{\ell} \sum_{k} \sum_{j}\left|Q_{k}\left(\lambda_{\ell}^{\prime}\right)\right|\left|Q_{j}\left(\lambda_{\ell}^{\prime}\right)\right| \sqrt{\left\langle E_{\sqrt{L}}\left(\lambda_{\ell}, \lambda_{\ell+1}\right] f_{k}, f_{k}\right\rangle} \sqrt{\left\langle E_{\sqrt{L}}\left(\lambda_{\ell}, \lambda_{\ell+1}\right] f_{j}, f_{j}\right\rangle} \\
& =\sum_{\ell}\left(\sum_{k}\left|Q_{k}\left(\lambda_{\ell}^{\prime}\right)\right| \sqrt{\left\langle E_{\sqrt{L}}\left(\lambda_{\ell}, \lambda_{\ell+1}\right] f_{k}, f_{k}\right\rangle}\right)^{2} \\
& \leq \sum_{\ell}\left(\sum_{k}\left|Q_{k}\left(\lambda_{\ell}^{\prime}\right)\right|^{2}\right) \sum_{k}\left\langle E_{\sqrt{L}}\left(\lambda_{\ell}, \lambda_{\ell+1}\right] f_{k}, f_{k}\right\rangle \\
& \leq C \sum_{\ell} \sum_{k}\left\langle E_{\sqrt{L}}\left(\lambda_{\ell}, \lambda_{\ell+1}\right] f_{k}, f_{k}\right\rangle \\
& \leq C \sum_{k}\left\|f_{k}\right\|_{2}^{2}
\end{aligned}
$$

where we used the fact that $\sum_{k}\left|Q_{k}(\lambda)\right|^{2} \leq C$.

Proof of Theorem 5.1. Assume that condition $\left(\mathrm{ST}_{\mathrm{p}, 2}^{\mathrm{q}}\right)$ holds for some $1 \leq p<2$ and $1 \leq q \leq \infty$. Fix a $f \in L^{p}$ and $\alpha>\mu(X)^{-1 / p}\|f\|_{p}$, and apply the Calderón-Zygmund decomposition at height $\alpha$ to $|f|^{p}$. There exist constants $C$ and $K$ so that

(i) $f=g+b=g+\sum_{j} b_{j}$;

(ii) $\|g\|_{p} \leq C\|f\|_{p},\|g\|_{\infty} \leq C \alpha$;

(iii) $b_{j}$ is supported in $B_{j}$ and $\#\left\{j: x \in 4 B_{j}\right\} \leq K$ for all $x \in X$;

(iv) $\int_{X}\left|b_{j}\right|^{p} d \mu \leq C \alpha^{p} \mu\left(B_{j}\right)$, and $\sum_{j} \mu\left(B_{j}\right) \leq C \alpha^{-p}\|f\|_{p}^{p}$.

Note that by (ii) $\alpha^{p-2}\|g\|_{2}^{2} \leq C\|f\|_{p}^{p}$.

Let $r_{B_{j}}$ be the radius of $B_{j}$ and denote by $J_{k}=\left\{j: 2^{k} / R \leq r_{B_{j}}<2^{k+1} / R\right\}$. Write

$$
f=g+\sum_{j} b_{j}=g+\sum_{k \leq 0} \sum_{j \in J_{k}} b_{j}+\sum_{k>0} \sum_{j \in J_{k}} b_{j}=g+h_{1}+h_{2} .
$$


By a standard argument it is enough to show that there exists a constant $C>0$ independent of $R$ and $\alpha$ such that for every $\alpha>\mu(X)^{-1 / p}\|f\|_{p}$,

$$
\mu\left(\left\{x: S_{R}^{\delta_{q}(p)}(L)(g)(x)>\alpha\right\}\right) \leq C \alpha^{-p}\|f\|_{p}^{p}
$$

and that for $i=1,2$,

$$
\mu\left(\left\{x: S_{R}^{\delta_{q}(p)}(L)\left(h_{i}\right)(x)>\alpha\right\}\right) \leq C \alpha^{-p}\|f\|_{p}^{p}
$$

Note that $\sup _{\lambda, R>0}\left(1-\frac{\lambda}{R}\right)_{+}^{\delta_{q}(p)}=1$ and that by by (ii) $\alpha^{p-2}\|g\|_{2}^{2} \leq C\|f\|_{p}^{p}$. Hence by spectral theory

$$
\begin{aligned}
\mu\left(\left\{x: S_{R}^{\delta_{q}(p)}(L)(g)(x)>\alpha\right\}\right) & \leq \alpha^{-2}\left\|S_{R}^{\delta_{q}(p)}(L)(g)\right\|_{2}^{2} \leq \alpha^{-2}\|g\|_{2}^{2} \\
& \leq C \alpha^{-p}\|f\|_{p}^{p} .
\end{aligned}
$$

Next we prove (5.4) for $i=1$. By the spectral theorem and equality (5.1)

$$
\begin{aligned}
\sum_{k \leq 0} \sum_{j \in J_{k}} S_{R}^{\delta_{q}(p)}(L) b_{j} & =\sum_{k \leq 0} \eta_{k}(\sqrt{L})\left(\sum_{j \in J_{k}} n_{k}(\sqrt{L}) b_{j}\right) \\
& +S_{R}^{\delta_{q}(p)}(L)\left(\sum_{k \leq 0} \sum_{j \in J_{k}} n_{k}(\sqrt{L}) b_{j}\right) .
\end{aligned}
$$

Applying the spectral theorem and Lemma 5.5 with $Q_{k}(\lambda)=\eta_{k}(\lambda)$ yields

$$
\left\|\sum_{k \leq 0} \sum_{j \in J_{k}} S_{R}^{\delta_{q}(p)}(L) b_{j}\right\|_{2}^{2} \leq C \sum_{k \leq 0}\left\|\sum_{j \in J_{k}} n_{k}(\sqrt{L}) b_{j}\right\|_{2}^{2}+C\left\|\sum_{k \leq 0} \sum_{j \in J_{k}} n_{k}(\sqrt{L}) b_{j}\right\|_{2}^{2} .
$$

Next supp $\widehat{n_{k}} \subseteq\left[-2^{k} / R, 2^{k} / R\right]$ so by Lemma 2.1 ,

$$
\operatorname{supp} K_{n_{k}(\sqrt{L})} \subseteq\left\{(x, y) \in X \times X: d(x, y) \leq 2^{k} / R\right\} .
$$

Hence if $j \in J_{k}$, then $\operatorname{supp} n_{k}(\sqrt{L}) b_{j} \subseteq 4 B_{j}$. Thus by (iii) there exists constant $C>0$ such that

$$
\sum_{k \leq 0}\left\|\sum_{j \in J_{k}} n_{k}(\sqrt{L}) b_{j}\right\|_{2}^{2}+\left\|\sum_{k \leq 0} \sum_{j \in J_{k}} n_{k}(\sqrt{L}) b_{j}\right\|_{2}^{2} \leq C \sum_{k \leq 0} \sum_{j \in J_{k}}\left\|n_{k}(\sqrt{L}) b_{j}\right\|_{2}^{2} .
$$

Next, by Proposition 2.3 and Remark 2.2,

$$
\begin{aligned}
\left\|n_{k}(\sqrt{L}) b_{j}\right\|_{2} & =\left\|n_{k}(\sqrt{L}) P_{B_{j}} b_{j}\right\|_{2} \\
& \leq\left\|n_{k}(\sqrt{L}) P_{B_{j}}\right\|_{p \rightarrow 2}\left\|b_{j}\right\|_{p} \\
& \leq\left\|n_{k}(\sqrt{L})\left(I+2^{k} \frac{\sqrt{L}}{R}\right)^{N}\right\|_{2 \rightarrow 2}\left\|\left(I+2^{k} \frac{\sqrt{L}}{R}\right)^{-N} P_{B_{j}}\right\|_{p \rightarrow 2}\left\|b_{j}\right\|_{p} \\
& \leq C\left\|\left(I+2^{k} \frac{\sqrt{L}}{R}\right)^{-N} P_{B_{j}}\right\|_{p \rightarrow 2}\left\|b_{j}\right\|_{p} \\
& \leq C \mu\left(B_{j}\right)^{\frac{1}{2}-\frac{1}{p}} \alpha \mu\left(B_{j}\right)^{\frac{1}{p}} \\
& \leq C \alpha \mu\left(B_{j}\right)^{1 / 2} .
\end{aligned}
$$

Hence by (iv)

$$
\begin{aligned}
\mu\left(\left\{x:\left|S_{R}^{\delta_{q}(p)}(L)\left(\sum_{k \leq 0} \sum_{j \in J_{k}} b_{j}\right)\right|>\alpha\right\}\right) & \leq C \alpha^{-2}\left\|S_{R}^{\delta_{q}(p)}(L)\left(\sum_{k \leq 0} \sum_{j \in J_{k}} b_{j}\right)\right\|_{2}^{2} \\
& \leq C \alpha^{-p}\|f\|_{p}^{p}
\end{aligned}
$$


Now, we prove (5.4) for $i=2$. Let $\Omega^{*}=\bigcup_{j \in \mathbb{N}} 4 B_{j}$. By (2.1) that

$$
\mu\left(\Omega^{*}\right) \leq C \sum_{j} \mu\left(B_{j}\right) \leq C \alpha^{-p}\|f\|_{p}^{p}
$$

Hence it is enough to show that

$$
\left\|S_{R}^{\delta_{q}(p)}(L)\left(\sum_{k>0} \sum_{j \in J_{k}} b_{j}\right)\right\|_{L^{2}\left(X \backslash \Omega^{*}\right)}^{2} \leq C \alpha^{2} \sum_{j} \mu\left(B_{j}\right) \leq C \alpha^{2-p}\|f\|_{p}^{p} .
$$

Using the decomposition from Lemma 5.4 we write

$$
S_{R}^{\delta_{q}(p)}(L)\left(\sum_{k>0} \sum_{j \in J_{k}} b_{j}\right)=\sum_{k>0} \sum_{j \in J_{k}} m_{k}(\sqrt{L}) b_{j}+\sum_{k>0} \eta_{k}(\sqrt{L}) n_{k}(\sqrt{L})\left(\sum_{j \in J_{k}} b_{j}\right) .
$$

Recall that $\widehat{m_{k}}$ is even and supported in $\left[-2^{k} / R, 2^{k} / R\right]$. By Lemma 2.1

$$
\operatorname{supp} K_{m_{k}(\sqrt{L})} \subset\left\{(x, y) \in X \times X: d(x, y) \leq \frac{2^{k}}{R}\right\} .
$$

This implies that if $x \in X \backslash \Omega^{*}$, then $m_{k}(\sqrt{L}) b_{j}(x)=0$ for any $j \in J_{k}$ and $k>0$ so it makes no contribution to (5.8). By (5.9) and Lemma 5.5

$$
\begin{aligned}
\left\|S_{R}^{\delta_{q}(p)}(L)\left(\sum_{k>0} \sum_{j \in J_{k}} b_{j}\right)\right\|_{L^{2}\left(X \backslash \Omega^{*}\right)}^{2} & \leq\left\|\sum_{k>0} \eta_{k}(\sqrt{L}) n_{k}(\sqrt{L})\left(\sum_{j \in J_{k}} b_{j}\right)\right\|_{2}^{2} \\
& \leq C \sum_{k>0}\left\|n_{k}(\sqrt{L})\left(\sum_{j \in J_{k}} b_{j}\right)\right\|_{2}^{2} .
\end{aligned}
$$

Next $\widehat{n_{k}}$ is even and supported on $\left[-2^{k} / R, 2^{k} / R\right]$ so by Lemma 2.1

$$
\operatorname{supp} K_{n_{k}(\sqrt{L})} \subseteq\left\{(x, y) \in X \times X: d(x, y) \leq 2^{k} / R\right\} .
$$

Hence $\operatorname{supp} n_{k}(\sqrt{L}) b_{j} \subseteq 4 B_{j}$ for $j \in J_{k}$. By (iii) there exists a constant $C>0$ such that

$$
\sum_{k>0}\left\|n_{k}(\sqrt{L})\left(\sum_{j \in J_{k}} b_{j}\right)\right\|_{2}^{2} \leq C \sum_{k>0} \sum_{j \in J_{k}}\left\|n_{k}(\sqrt{L}) b_{j}\right\|_{2}^{2} .
$$

To continue, fix $\psi \in C_{c}^{\infty}$, even and supported in $[-2,2]$ such that $\psi(\lambda)=1$ for $|\lambda| \leq 3 / 2$. Write

$$
n_{k}(\sqrt{L})=n_{k}(\sqrt{L}) \psi\left(\frac{\sqrt{L}}{R}\right)+n_{k}(\sqrt{L})\left(1-\psi\left(\frac{\sqrt{L}}{R}\right)\right) .
$$

Then $\operatorname{supp}(1-\psi(\lambda / R)) \subset(-\infty,-3 R / 2] \cup[3 R / 2, \infty)$ so for every $k>0$ and $\delta_{q}(p) \geq 0$,

$$
\begin{aligned}
\left|n_{k}(\lambda)\left(1-\psi\left(\frac{\lambda}{R}\right)\right)\right| & \leq C\left|1-\psi\left(\frac{\lambda}{R}\right)\right| 2^{-\delta_{q}(p) k}\left(1+2^{k}\left|1-\frac{|\lambda|}{R}\right|\right)^{-N} \\
& \leq C\left(1+\left|\frac{2^{k} \lambda}{R}\right|\right)^{-N},
\end{aligned}
$$

and we use a similar argument as in the proof of (5.4) for $i=1$ to conclude that

$$
\left\|n_{k}(\sqrt{L})\left(I-\psi\left(\frac{\sqrt{L}}{R}\right)\right) b_{j}\right\|_{2} \leq C \alpha \mu\left(B_{j}\right)^{1 / 2} .
$$

On the other hand, $\operatorname{supp} n_{k}(\lambda) \psi(\lambda / R) \subset[-2 R, 2 R]$ so by $\left(\mathrm{ST}_{\mathrm{p}, 2}^{\mathrm{q}}\right)$

$$
\left\|n_{k}(\sqrt{L}) \psi\left(\frac{\sqrt{L}}{R}\right) b_{j}\right\|_{2}=\left\|n_{k}(\sqrt{L}) \psi\left(\frac{\sqrt{L}}{R}\right) P_{B_{j}} b_{j}\right\|_{2}
$$




$$
\begin{aligned}
& \leq\left\|n_{k}(\sqrt{L}) \psi\left(\frac{\sqrt{L}}{R}\right) P_{B_{j}}\right\|_{p \rightarrow 2}\left\|b_{j}\right\|_{p} \\
& \leq \operatorname{C\alpha } \mu\left(B_{j}\right)^{\frac{1}{2}} 2^{k n\left(\frac{1}{p}-\frac{1}{2}\right)}\left\|\delta_{2 R}\left(n_{k}(\lambda) \psi(\lambda / R)\right)\right\|_{q} .
\end{aligned}
$$

Now

$$
\begin{aligned}
\left\|\delta_{2 R}\left(n_{k}(\lambda) \psi\left(\frac{\lambda}{R}\right)\right)\right\|_{q} & \leq C\left(\int_{0}^{1}\left|n_{k}(2 R \lambda)\right|^{q} d \lambda\right)^{1 / q} \\
& \leq C 2^{-\delta_{q}(p) k}\left(\int_{0}^{1}\left(1+2^{k}|1-2 \lambda|\right)^{-N q} d \lambda\right)^{1 / q} \\
& \leq C 2^{-\delta_{q}(p) k} 2^{-\frac{k}{q}}=C 2^{-n k\left(\frac{1}{p}-\frac{1}{2}\right)} .
\end{aligned}
$$

This yields

$$
\left\|n_{k}(\sqrt{L}) \psi\left(\frac{\sqrt{L}}{R}\right) b_{j}\right\|_{2} \leq C \alpha \mu\left(B_{j}\right)^{1 / 2} .
$$

By (5.13) and (5.14)

$$
\left\|n_{k}(\sqrt{L}) b_{j}\right\|_{2} \leq C \alpha \mu\left(B_{j}\right)^{1 / 2} .
$$

The rest of the proof of (5.4) for $i=2$ is similar to the case $i=1$.

Proof of Theorem 5.2. Assume that condition $\left(\mathrm{SC}_{\mathrm{p}, 2}^{\mathrm{q}, 1}\right)$ holds for some $1 \leq p<2$ and $1 \leq q \leq \infty$. The proof of Theorem 5.2 is almost identical to that of Theorem 5.1 except some minor technical complications so we only give a brief sketch of it. tion

We apply the Calderón-Zygmund decomposition at height $\alpha$ to $|f|^{p}$ to get the same decomposi-

$$
f=g+\sum_{k \leq 0} \sum_{j \in J_{k}} b_{j}+\sum_{k>0} \sum_{j \in J_{k}} b_{j}=g+h_{1}+h_{2}
$$

as in Theorem 5.1. The proof of week type estimates for $g$ and $h_{1}$ uses the simple observation that $\left(\mathrm{SC}_{\mathrm{p}, 2}^{\mathrm{q}, 1}\right) \Rightarrow\left(\mathrm{SC}_{\mathrm{p}, 2}^{\infty}\right) \Leftrightarrow\left(\mathrm{ST}_{\mathrm{p}, 2}^{\infty}\right) \Rightarrow\left(\mathrm{E}_{\mathrm{p}, 2}\right)$ (see Remark 3.11 and Proposition 2.3$)$ and is essentially the same as the corresponding argument in the proof of Theorem 5.1.

It remains to show that

$$
\mu\left(\left\{x: S_{R}^{\delta_{q}(p)}(L)\left(h_{2}\right)(x)>\alpha\right\}\right) \leq C \alpha^{-p}\|f\|_{p}^{p} .
$$

To show (5.15) we note that if $\mu(X)$ is finite, then we may assume $X=B\left(x_{0}, 1\right)$ for some $x_{0} \in X$. Thus the radius of each $B_{j}$ in the Calderón-Zygmund decomposition satisfies $2^{k} / R \leq 4$. If $R \leq 4$, then $k \leq 4$. Hence one can use the same argument as in the proof of Theorem 5.1 for $i=1$.

Next we consider the remaining case $R>4$. Using the decomposition described in Lemma 5.4 it is not difficult to note that to finish the proof it is enough to show that

$$
\begin{aligned}
\left\|n_{k}(\sqrt{L})\left(I-\psi\left(\frac{\sqrt{L}}{R}\right)\right) b_{j}\right\|_{2} & \leq C \alpha \mu\left(B_{j}\right)^{1 / 2}, \\
\left\|n_{k}(\sqrt{L}) \psi\left(\frac{\sqrt{L}}{R}\right) b_{j}\right\|_{2} & \leq C \alpha \mu\left(B_{j}\right)^{1 / 2},
\end{aligned}
$$

where $n_{k}$ is defined in Lemma 5.4 and $\psi$ is a function in (5.12). The proof of (5.16) is similar to that of (5.13). 
To prove (5.17) set $N=[2 R]+1$. By condition $\left(\mathrm{SC}_{\mathrm{p}, 2}^{\mathrm{q}, 1}\right)$

$$
\left\|n_{k}(\sqrt{L}) \psi\left(\frac{\sqrt{L}}{R}\right) b_{j}\right\|_{2} \leq C \mu\left(B_{j}\right)^{\left(\frac{1}{2}-\frac{1}{p}\right)} 2^{k n\left(\frac{1}{p}-\frac{1}{2}\right)}\left\|\delta_{N}\left(n_{k}(\lambda) \psi(\lambda / R)\right)\right\|_{N, q}\left\|b_{j}\right\|_{p}
$$

Next (assuming that $\sup |\psi|=1$ )

$$
\begin{aligned}
& 2^{1 / q}\left\|\delta_{N}\left(n_{k}(\lambda) \psi(\lambda / R)\right)\right\|_{N, q} \leq\left(\frac{1}{N} \sum_{\ell=1-N}^{N} \sup _{\lambda \in[\ell-1, \ell)}\left|n_{k}(\lambda)\right|^{q}\right)^{1 / q} \\
& \leq\left(\frac{1}{N} \sum_{\ell=1-N}^{-[R]-2} \sup _{\lambda \in[\ell-1, \ell)}\left|n_{k}(\lambda)\right|^{q}\right)^{1 / q}+\left(\frac{1}{N} \sum_{\ell=-[R]-1}^{-[R]+3} \sup _{\lambda \in[\ell-1, \ell)}\left|n_{k}(\lambda)\right|^{q}\right)^{1 / q} \\
&+\left(\frac{1}{N} \sum_{\ell=-[R]+4}^{0} \sup _{\lambda \in[\ell-1, \ell)}\left|n_{k}(\lambda)\right|^{q}\right)^{1 / q}+\left(\frac{1}{N} \sum_{\ell=1}^{[R]-3} \sup _{\lambda \in[\ell-1, \ell)}\left|n_{k}(\lambda)\right|^{q}\right)^{1 / q} \\
&+\left(\frac{1}{N} \sum_{\ell=[R]-2}^{[R]+2} \sup _{\lambda \in[\ell-1, \ell)}\left|n_{k}(\lambda)\right|^{q}\right)^{1 / q}+\left(\frac{1}{N} \sum_{\ell=[R]+3}^{N} \sup _{\lambda \in[\ell-1, \ell)}\left|n_{k}(\lambda)\right|^{q}\right)^{1 / q}=I+I I+I I I+I V+V+V I .
\end{aligned}
$$

Let $M$ be a sufficiently large natural number. By Lemma 5.4

$$
\begin{aligned}
V & =\left(\frac{1}{N} \sum_{\ell=[R]-2}^{[R]+2} \sup _{\lambda \in[\ell-1, \ell)}\left|n_{k}(\lambda)\right|^{q}\right)^{1 / q} \\
& \leq C 2^{-\delta_{q}(p) k}\left(\frac{1}{N} \sum_{\ell=[R]-2}^{[R]+2} \sup _{\lambda \in[\ell-1, \ell)}\left(1+2^{k}\left|\frac{\lambda}{R}-1\right|\right)^{-M q}\right)^{1 / q} \\
& \leq C 2^{-\delta_{q}(p) k} R^{-1 / q} \leq C 2^{-\delta_{q}(p) k} 2^{-\frac{k}{q}}\left(\frac{2^{k}}{R}\right)^{1 / q} \\
& \leq C 2^{-n k\left(\frac{1}{p}-\frac{1}{2}\right)}
\end{aligned}
$$

and

$$
\begin{aligned}
V I & \leq C\left(\frac{1}{N} \sum_{\ell=[R]+3}^{N} \sup _{\lambda \in[\ell-1, \ell)}\left|2^{-\delta_{q}(p) k}\left(1+2^{k}\left(\frac{\lambda}{R}-1\right)\right)^{-M}\right|^{q}\right)^{1 / q} \\
& \leq C 2^{-\delta_{q}(p) k}\left(\int_{R}^{\infty}\left(1+2^{k}\left(\frac{\lambda}{R}-1\right)\right)^{-M q} R^{-1} d \lambda\right)^{1 / q} \\
& \leq C 2^{-n k\left(\frac{1}{p}-\frac{1}{2}\right)}
\end{aligned}
$$

A similar argument as in $V$ shows that $I I \leq C 2^{-n k(1 / p-1 / 2)}$; the similar argument as in $V I$ shows that each of $I$, III and $I V$ is less than $C 2^{-n k(1 / p-1 / 2)}$. Thus

$$
\left\|\delta_{N}\left(n_{k}(\lambda) \psi(\lambda / R)\right)\right\|_{N, q} \leq C 2^{-n k\left(\frac{1}{p}-\frac{1}{2}\right)} .
$$

This finishes the proof of Theorem 5.2. 


\section{Part 2. Dispersive and restriction estimates}

\section{DisPersive and STRIChartZ ESTIMATES}

Let $(X, d, \mu)$ be a metric measure space. Next let $L$ be a non-negative self-adjoint operator acting on $L^{2}(X)$. In virtue of the spectral theory, we can define the semigroup $\exp (-z L)$ for all $z \in \mathbb{C}$ with $\operatorname{Re} z \geq 0$ and such that

$$
\|\exp (-z L)\|_{2 \rightarrow 2} \leq 1
$$

We say that the operator $L$ satisfies a dispersive type estimates if there exist constants $n$ and $C$ such that

$$
\|\exp (i s L)\|_{1 \rightarrow \infty} \leq C|s|^{-n / 2}, \quad \forall s \in \mathbb{R} \backslash\{0\} .
$$

Of course, the standard Laplacian on $\mathbb{R}^{n}$ satisfies the dispersive estimate. Such estimates are of importance in analysis and PDE. In particular, they imply endpoint Strichartz estimates (see Keel and Tao [37]). We refer to Strichartz endpoint estimates for the corresponding Schrödinger equation as

$$
\int_{\mathbb{R}}\left\|e^{i t L} f\right\|_{\frac{2 n}{n-2}}^{2} d t \leq C\|f\|_{2}^{2}, f \in L^{2}
$$

This endpoint estimate together with the obvious fact

$$
\|\exp (i t L) f\|_{L_{t}^{\infty} L_{x}^{2}} \leq\|f\|_{L^{2}}
$$

give $L_{t}^{p} L_{x}^{q}$ Strichartz estimates. See [37] for more details. Our aim will be to explain how sharp spectral multipliers follow from dispersive or Strichartz estimates.

It is natural to consider the dispersive estimate (6.1) in conjunction with the smoothing condition

$$
\|\exp (-t L)\|_{1 \rightarrow \infty} \leq K t^{-n / 2}, \forall t>0 .
$$

Note that if the self-adjoint contractive semigroup $\exp (-t L)$ on $L^{2}(X)$ is in addition uniformly bounded on $L^{\infty}(X)$, which includes the case of a sub-Markovian semigroup, then

$$
\|\exp (-(t+i s) L)\|_{1 \rightarrow \infty} \leq\|\exp (-t L / 2)\|_{\infty \rightarrow \infty}\|\exp (-i s L)\|_{1 \rightarrow \infty} \leq C|s|^{-n / 2} .
$$

Together with (6.3) this yields

$$
\|\exp (-(t+i s) L)\|_{1 \rightarrow \infty} \leq C \min \left\{t^{-n / 2},|s|^{-n / 2}\right\} \leq C^{\prime}|t+i s|^{-n / 2}
$$

for all $t>0, s \in \mathbb{R}$. Hence

$$
\|\exp (-z L)\|_{1 \rightarrow \infty} \leq C|z|^{-n / 2}
$$

for all $\operatorname{Re} z \geq 0$. Of course this estimate implies (6.1) and (6.3) and the argument above shows that if semigroup $\exp (-t L)$ is uniformly bounded on $L^{\infty}(X)$ then it is equivalent to conjunction (6.1) and (6.3). It turns out however that this equivalence holds without the boundedness assumption on $L^{\infty}(X)$. This fact will be used in the next subsection in which we will not assume uniform boundedness of semigroup $\exp (-t L)$ on $L^{\infty}(X)$.

Lemma 6.1. Suppose that $L$ is a non-negative self-adjoint operator on $L^{2}(X)$. Then the dispersive estimate (6.1) is equivalent to (6.4).

Proof. All what we need is to prove that (6.1) is enough to get (6.4) on the positive half-plane. Fix $f, g \in L^{1}(X) \cap L^{2}(X)$ and consider the function

$$
H(z)=z^{n / 2}\langle\exp (-z L) f, g\rangle .
$$


The analyticity of the semigroup on $L^{2}$ implies analyticity of $H$ on the open right half-plane and continuous on the boundary. Now for $z=i s$ with $s \in \mathbb{R}$, the dispersive estimate (6.1) gives

$$
|H(i s)| \leq C\|g\|_{1}\|f\|_{1} \text {. }
$$

For all $z$ with $\operatorname{Re} z \geq 0$, we have

$$
|H(z)| \leq|z|^{n / 2}\|g\|_{2}\|f\|_{2} .
$$

Therefore, we can apply the Phragmén-Lindelöf theorem and conclude that

$$
|H(z)| \leq C\|g\|_{1}\|f\|_{1}
$$

for all $z$ with $\operatorname{Re} z \geq 0$. From this and the density of $L^{1}(X) \cap L^{2}(X)$ in $L^{1}(X)$ we obtain the lemma.

\section{From dispersive and Strichartz ESTIMATES to ShaRP MULTIPLIERS}

We continue with the assumption that $(X, d, \mu)$ is a metric measure space. In this section, let us start with the following proposition.

Proposition 7.1. Let $L$ be a non-negative self-adjoint operator on $L^{2}$. Assume that $L$ satisfies the dispersive estimate (6.1). Then for all $1 \leq p<\frac{2 n}{n+2}$ and all $\lambda \geq 0$

$$
\left\|d E_{\sqrt{L}}(\lambda)\right\|_{p \rightarrow p^{\prime}} \leq C \lambda^{n\left(\frac{1}{p}-\frac{1}{p^{\prime}}\right)-1},
$$

where $p^{\prime}$ is again the conjugate exponent of $p$.

Proof. We first prove that $L$ satisfies

$$
\|F(\sqrt{L})\|_{p \rightarrow p^{\prime}} \leq C R^{n\left(\frac{1}{p}-\frac{1}{p^{\prime}}\right)}\left\|\delta_{R} F\right\|_{1}
$$

for all bounded $F \in L^{1}$ with $\operatorname{supp} F \subseteq[0, R]$ and all $p$ with $1 \leq p<\frac{2 n}{n+2}$. This estimate is very similar to $\left(\mathrm{ST}_{\mathrm{p}, \mathrm{p}^{\prime}}^{1}\right)$ studied in Part 1 . Note that we do not consider here $X$ to be a doubling space (even a metric $d$ is not needed).

Consider the case where $R=1$ and fix $F$ with support contained in $[0,1]$. Set $G(\lambda)=F(\sqrt{\lambda}) e^{\lambda}$. By the inverse Fourier transform, we have (up to a constant)

$$
G(\lambda)=\int_{\mathbb{R}} \hat{G}(\xi) e^{i \xi \lambda} d \xi
$$

This gives

$$
F(\sqrt{L})=\int_{\mathbb{R}} \hat{G}(\xi) \exp (-(1-i \xi) L) d \xi .
$$

This equality follows immediately from Fubini's theorem if $\hat{G} \in L^{1}$. One may start by proving (7.3) for smooth functions $F_{n}$ and then use standard approximation arguments to obtain the equality for all $F$ as above.

Now the dispersive estimate together with Lemma 6.1 imply that for any $p \in[1,2]$

$$
\begin{aligned}
\|F(\sqrt{L})\|_{p \rightarrow p^{\prime}} & \leq \int_{\mathbb{R}}\left|\hat{G}(\xi)\|\mid \exp (-(1-i \xi) L)\|_{p \rightarrow p^{\prime}} d \xi\right. \\
& \leq C \int_{\mathbb{R}}|\hat{G}(\xi)|\left(1+\xi^{2}\right)^{-\frac{n}{4}\left(\frac{1}{p}-\frac{1}{p^{\prime}}\right)} d \xi \\
& \leq C\|\hat{G}\|_{\infty} \int_{\mathbb{R}}\left(1+\xi^{2}\right)^{-\frac{n}{4}\left(\frac{1}{p}-\frac{1}{p^{\prime}}\right)} d \xi .
\end{aligned}
$$


Now we note that

$$
\|\hat{G}\|_{\infty} \leq\|G\|_{1} \leq C\|F\|_{1}
$$

and

$$
\int_{\mathbb{R}}\left(1+\xi^{2}\right)^{-\frac{n}{4}\left(\frac{1}{p}-\frac{1}{p^{\prime}}\right)} d \xi<\infty
$$

for $p<\frac{2 n}{n+2}$. This shows (7.2) when $R=1$. Now, for general $R>0$ and $F$ with support in $[0, R]$ we reproduce the previous arguments with the function $\delta_{R} F$ and the operator $L^{\prime}=\frac{L}{R^{2}}$. This leads to (7.2). Now we argue as in the proof of Proposition 2.4. Fix $\lambda \geq 0$ and $\varepsilon>0$ small. We use (7.2) to obtain

$$
\begin{aligned}
\left\|\varepsilon^{-1} E_{\sqrt{L}}(\lambda-\varepsilon, \lambda+\varepsilon]\right\|_{p \rightarrow p^{\prime}} & =\varepsilon^{-1}\left\|\mathbb{1}_{(\lambda-\varepsilon, \lambda+\varepsilon]}(\sqrt{L})\right\|_{p \rightarrow p^{\prime}} \\
& \leq C \varepsilon^{-1}(\lambda+\varepsilon)^{n\left(\frac{1}{p}-\frac{1}{p^{\prime}}\right)}\left\|\chi_{\left(\frac{\lambda-\varepsilon}{\lambda+\varepsilon}, 1\right]}\right\|_{1} \\
& \leq C(\lambda+\varepsilon)^{n\left(\frac{1}{p}-\frac{1}{p^{\prime}}\right)-1} .
\end{aligned}
$$

Letting $\varepsilon \rightarrow 0$ we obtain

$$
\left\|d E_{\sqrt{L}}(\lambda)\right\|_{p \rightarrow p^{\prime}} \leq C \lambda^{n\left(\frac{1}{p}-\frac{1}{p^{\prime}}\right)-1},
$$

which implies the estimate of the proposition.

Corollary 7.2. Suppose that $(X, d, \mu)$ satisfies the doubling property (2.2) and there exists a positive constant $C>0$ such that $V(x, r) \leq C r^{n}$ for every $x \in X$ and $r>0$. Assume that $L$ satisfies the finite speed propagation property (FS) and the dispersive estimate (6.1). Fix $p \in\left[1, \frac{2 n}{n+2}\right]$ and suppose next that $G$ is a distribution, $\operatorname{supp} G \subset[0, R]$ and that $\int_{\mathbb{R}}|\hat{G}(\xi)|\left(1+\xi^{2} / R^{2}\right)^{-\frac{n}{4}\left(\frac{1}{p}-\frac{1}{p^{\prime}}\right)} d \xi<\infty$. Then the operator $G(L)$ is well defined as an operator acting from $L^{p}$ to $L^{p^{\prime}}$ and

$$
\|G(L)\|_{p \rightarrow p^{\prime}} \leq C \int_{\mathbb{R}}|\hat{G}(\xi)|\left(1+\xi^{2} / R^{2}\right)^{-\frac{n}{4}\left(\frac{1}{p}-\frac{1}{p^{\prime}}\right)} d \xi .
$$

Proof. Corollary 7.2 follows from estimates (7.4).

Proposition 7.1 does not yield the optimal results for the standard Laplace operator. However in the abstract setting we can include the endpoint $p=\frac{2 n}{n+2}$ when $n>2$ in the following way. We start with the Strichartz estimate (6.2) and repeat the previous proof to get

$$
\begin{aligned}
\|F(\sqrt{L})\|_{2 \rightarrow \frac{2 n}{n-2}} & \leq \int_{\mathbb{R}} \mid \hat{G}(\xi)\|\exp (-(1-i \xi) L)\|_{2 \rightarrow \frac{2 n}{n-2}} d \xi \\
& \leq\|\hat{G}\|_{2}\left(\int_{\mathbb{R}}\|\exp (i \xi L)\|_{2 \rightarrow \frac{2 n}{n-2}}^{2} d \xi\right)^{1 / 2} \\
& \leq C\|F\|_{2}
\end{aligned}
$$

for all $F$ with support in $[0,1]$. For $F$ supported in $[0, R]$ we apply the previous estimate with $\delta_{R} F$ and $L^{\prime}=\frac{L}{R^{2}}$ to get

$$
\|F(\sqrt{L})\|_{2 \rightarrow \frac{2 n}{n-2}} \leq C R\left\|\delta_{R} F\right\|_{2}
$$

Therefore,

$$
\|F(\sqrt{L})\|_{\frac{2 n}{n+2} \rightarrow \frac{2 n}{n-2}} \leq C R^{2}\left\|\delta_{R} F\right\|_{2}^{2} .
$$

Similar arguments as above give (7.1) for $p=\frac{2 n}{n+2}$. 
We can now extend this easily to $p<\frac{2 n}{n+2}$ if the smoothing property (6.3) is satisfied. More precisely, fix $p<\frac{2 n}{n+2}$ and assume that

$$
\|\exp (-t L)\|_{p \rightarrow \frac{2 n}{n+2}} \leq K t^{-\frac{n}{2}\left(\frac{1}{p}-\frac{n+2}{2 n}\right)}, \forall t>0 .
$$

We introduce as before $G_{R}(\lambda)=\left(\delta_{R} F\right)(\sqrt{\lambda}) e^{\lambda}$ for $F$ supported in $[0, R]$. Then for $q=p^{\prime}$ we have

$$
\begin{aligned}
\|F(\sqrt{L}) f\|_{q} & =\left\|\int_{\mathbb{R}} \widehat{G_{R}}(\xi) \exp \left(-\left(\frac{1}{R^{2}}-i \frac{\xi}{R^{2}}\right) L\right) f d \xi\right\|_{q} \\
& \leq\left\|\exp \left(-\frac{L}{R^{2}}\right)\right\|_{\frac{2 n}{n-2} \rightarrow q} \int_{\mathbb{R}} \widehat{\mid G_{R}}(\xi)\left\|\exp \left(-\left(i \frac{\xi}{R^{2}}\right) L\right) f\right\|_{\frac{2 n}{n-2}} d \xi \\
& \leq C R^{n\left(\frac{1}{2}-\frac{1}{q}\right)-1}\left\|G_{R}\right\|_{2}\left(\int_{\mathbb{R}}\left\|\exp \left(-\left(i \frac{\xi}{R^{2}}\right) L\right) f\right\|_{\frac{2 n}{n-2}}^{2} d \xi\right)^{1 / 2} \\
& \leq C R^{n\left(\frac{1}{2}-\frac{1}{q}\right)}\left\|\delta_{R} F\right\|_{L^{2}(\mathbb{R})}\|f\|_{L^{2}(X)},
\end{aligned}
$$

where we used the Strichartz estimate (6.2) to obtain the last inequality. As in the last proposition, this gives the Stein-Tomas restriction (7.1) for all $p \in\left[1, \frac{2 n}{n+2}\right]$. We have proved

Proposition 7.3. Let $L$ be a non-negative self-adjoint operator on $L^{2}$. Assume that $L$ satisfies the Strichartz estimate (6.2) for some $n>2$. Fix $p$ such that $1 \leq p \leq \frac{2 n}{n+2}$ and assume that the smoothing property (7.6) is satisfied. Then for all $\lambda \geq 0$

$$
\left\|d E_{\sqrt{L}}(\lambda)\right\|_{p \rightarrow p^{\prime}} \leq C \lambda^{n\left(\frac{1}{p}-\frac{1}{p^{\prime}}\right)-1},
$$

where $p^{\prime}$ is again the conjugate exponent of $p$.

We mentioned above that the dispersive estimate implies the Strichartz estimate ([37]). For this reason we formulate the results below for the case where $L$ satisfies the endpoint Strichartz estimate.

Theorem 7.4. Suppose that $(X, d, \mu)$ satisfies the doubling property (2.2) and there exists a positive constant $C>0$ such that $V(x, r) \leq C r^{n}$ for every $x \in X$ and $r>0$. Assume that $L$ satisfies the finite speed propagation property (FS) and the Strichartz estimate (6.2) with the same $n$ as in the doubling property. Assume also that $n>2$. Fix $p \in\left[1, \frac{2 n}{n+2}\right]$ and assume that (7.6) holds. For every compactly supported bounded function $F$ such that

$$
\|F\|_{W^{\beta, 2}}<\infty
$$

for some $\beta>n\left(\frac{1}{p}-\frac{1}{2}\right)$, the operator $F(t \sqrt{L})$ is bounded on $L^{p}$ for all $t>0$ and

$$
\sup _{t>0}\|F(t \sqrt{L})\|_{p \rightarrow p} \leq C\|F\|_{W^{\beta, 2}} .
$$

Proof. If $F$ is supported in $[0, R]$ then by Proposition 7.3, we have

$$
\begin{aligned}
\|F(\sqrt{L})\|_{p \rightarrow p^{\prime}} & =\left\|\int_{0}^{R} F(\lambda) d E_{\sqrt{L}}(\lambda)\right\|_{p \rightarrow p^{\prime}} \\
& \leq C \int_{0}^{R}|F(\lambda)| \lambda^{n\left(\frac{1}{p}-\frac{1}{p^{\prime}}\right)-1} d \lambda \\
& \leq C R^{n\left(\frac{1}{p}-\frac{1}{p^{\prime}}\right)}\left\|\delta_{R} F\right\|_{1} .
\end{aligned}
$$

Hence by the $T^{*} T$ argument

$$
\|F(\sqrt{L})\|_{p \rightarrow 2} \leq C R^{n\left(\frac{1}{p}-\frac{1}{2}\right)}\left\|\delta_{R} F\right\|_{2}
$$


Combining this with our assumption on the volume yields $\left(\mathrm{ST}_{\mathrm{p}, 2}^{2}\right)$. We then apply Theorem 3.1 and obtain the result for $p \in\left[1, \frac{2 n}{n+2}\right]$.

We have seen that the assumptions of the previous theorem imply $\left(\mathrm{ST}_{\mathrm{p}, 2}^{2}\right)$, we can then apply Theorem 5.1 to obtain endpoint estimate for Bochner-Riesz means. In addition, by applying Theorem 4.1 we obtain under the assumptions of the previous theorem the following result.

Theorem 7.5. Fix $p \in\left[1, \frac{2 n}{n+2}\right]$. For any even bounded Borel function $F$ such that $\sup _{t>0}\left\|\eta \delta_{t} F\right\|_{W^{\beta, 2}}$ $<\infty$ for some $\beta>\max \{n(1 / p-1 / 2), 1 / 2\}$ and some non-trivial function $\eta \in C_{c}^{\infty}(0, \infty)$, the operator $F(\sqrt{L})$ is bounded on $L^{r}(X)$ for all $r \in\left(p, p^{\prime}\right)$. In addition,

$$
\|F(\sqrt{L})\|_{r \rightarrow r} \leq C_{\beta}\left(\sup _{t>0}\left\|\eta \delta_{t} F\right\|_{W^{\beta, 2}}+|F(0)|\right) .
$$

Remark 7.6. In the general setting of doubling spaces, we can replace the dispersive estimate (6.1) by

$$
\left\|P_{B(x, r)} \exp (i s L) P_{B(x, r)}\right\|_{p \rightarrow p^{\prime}} \leq C V(x, r)^{\frac{1}{p^{\prime}}-\frac{1}{p}}\left(\frac{r}{\sqrt{|s|}}\right)^{n\left(\frac{1}{p}-\frac{1}{p^{\prime}}\right)} .
$$

Here the constant $n$ is the same as in the doubling condition. The arguments in the proof of (7.2) show that for $p<\frac{2 n}{n+2}$,

$$
\left\|P_{B(x, r)}|F|^{2} P_{B(x, r)}\right\|_{p \rightarrow p^{\prime}} \leq C V(x, r)^{\frac{1}{p^{\prime}}-\frac{1}{p}}(R r)^{n\left(\frac{1}{p}-\frac{1}{p^{\prime}}\right)}\left\|\delta_{R} F\right\|_{2}^{2}
$$

for all $F$ supported in $[0, R]$. The $T^{*} T$ argument gives $\left(\mathrm{ST}_{\mathrm{p}, 2}^{2}\right)$ for all $p<\frac{2 n}{n+2}$. Therefore we obtain the same conclusion as in Theorems 7.4 and 7.5 for L satisfying property (FS) and (7.8) on any doubling space.

Note also that under these two assumptions, we obtain from Theorem 5.1 endpoint estimates for the Bochner-Riesz mean $S_{R}^{\delta(p)}(L)$ on $L^{p}\left(p \in\left[1, \frac{2 n}{n+2}\right)\right)$ for $\delta(p)=\max \left\{0, n\left|\frac{1}{p}-\frac{1}{2}\right|-\frac{1}{2}\right\}$.

In the following proposition we consider local dispersive estimate.

Proposition 7.7. Let $L$ be a non-negative self-adjoint operator on $L^{2}$. Assume that $L$ satisfies the local dispersive estimate

$$
\|\exp (i t L)\|_{1 \rightarrow \infty} \leq C t^{-n / 2}
$$

for all $0<|t| \leq 2$. Assume also that

$$
\|\exp (-t L)\|_{\infty \rightarrow \infty} \leq C \text { and }\|\exp (-t L)\|_{1 \rightarrow \infty} \leq C t^{-n / 2}
$$

for all $t>0$. Then for all $1 \leq p<\frac{2 n}{n+2}$ and all $k>0$

$$
\left\|E_{L}[k, k+1)\right\|_{p \rightarrow p^{\prime}} \leq C(1+k)^{\frac{n}{2}\left(\frac{1}{p}-\frac{1}{p^{\prime}}\right)-1}
$$

where $p^{\prime}$ is again the conjugate exponent of $p$.

Proof. Set $\widehat{G_{k}}(\xi)=3 \pi(1-|\xi|)_{+}^{2} e^{-i k \xi}$ so that $G_{k}(\lambda)=6(\lambda-k)^{-2}-6 \frac{\sin (\lambda-k)}{(\lambda-k)^{3}}$. Note that there exists a positive constant $c$ such that $G_{k}(\lambda) e^{-\lambda /(2 k)} \geq c \chi_{[k, k+1)}(\lambda)$ for all $\lambda \in[k, k+1)$. So

$$
c^{2}\left\|E_{L}[k, k+1) f\right\|_{2}^{2} \leq\left\|G_{k}(L) e^{-L /(2 k)} f\right\|_{2}^{2} .
$$

It follows from the above inequality and the $\mathrm{T}^{*} \mathrm{~T}$ argument that

$$
c^{2}\left\|E_{L}[k, k+1)\right\|_{p \rightarrow p^{\prime}} \leq\left\|\left.|| G_{k}\right|^{2}(L) e^{-L / k}\right\|_{p \rightarrow p^{\prime}} .
$$


Next (6.4) holds for $|\operatorname{Im} z| \leq 2$. Hence

$$
\begin{aligned}
\left\|\left|G_{k}\right|^{2}(L) e^{-L / k}\right\|_{p \rightarrow p^{\prime}} & \leq \int_{-2}^{2}\left|\widehat{G_{k}} * \widehat{G_{k}}\right| \mid \exp (-(1 / k-i \xi) L) \|_{p \rightarrow p^{\prime}} d \xi \\
& \leq C \int_{-2}^{2}\left(k^{-2}+\xi^{2}\right)^{-\frac{n}{4}\left(\frac{1}{p}-\frac{1}{p^{\prime}}\right)} d \xi \\
& \leq C \int_{\mathbb{R}}\left(k^{-2}+\xi^{2}\right)^{-\frac{n}{4}\left(\frac{1}{p}-\frac{1}{p^{\prime}}\right)} d \xi \\
& \leq C(1+k)^{\frac{n}{2}\left(\frac{1}{p}-\frac{1}{p^{\prime}}\right)-1} .
\end{aligned}
$$

This proves estimate (7.9).

\section{Part 3. Applications}

\section{Standard LAPLACE OPERATOR AND COMPACT MANifOLDS}

As mentioned in the introduction, the restriction estimates $\left(\mathrm{R}_{\mathrm{p}}\right)$ for standard Laplace operator on $\mathbb{R}^{n}$ are valid for $1 \leq p \leq 2(n+1) /(n+3)$. As a consequence of Theorem 5.1, we obtain alternative proof of Theorem 1.1 of [63] by Tao, Theorem 1 [15] and main result of [14] described by M. Christ. These results can be stated in the following way:

Proposition 8.1. For all $n \geq 2$ and $1 \leq p \leq 2(n+1) /(n+3)$, the operator $(I-\Delta)_{+}^{\delta(p)}$ is of weak-type $(p, p)$.

Proof. This result is straightforward from Proposition 2.4 and Theorem 5.1.

Similarly, using Theorem 5.2 one can obtain alternative proof of Theorem 1.2 of [63]. Our proof shows that this result holds for all operators on compact manifolds which satisfy property (FS) and condition $\left(\mathrm{S}_{\mathrm{p}}\right)$ as in the following proposition.

Proposition 8.2. Suppose the operator L satisfies (FS) and condition $\left(\mathrm{S}_{\mathrm{p}}\right)$ for some $1 \leq p \leq$ $2(n+1) /(n+3)$. Then the operator $\left(I-L / R^{2}\right)_{+}^{\delta(p)}$ is of weak-type $(p, p)$ uniformly in $R$.

Proof. This result is straightforward from Proposition 3.10 and Theorem 5.2.

In both cases of compact manifolds with or without boundaries, examples which satisfy condition $\left(S_{p}\right)$ are described in $[56,58]$ by C.D. Sogge.

We mentioned here endpoint Bochner-Riesz summability results. From Theorems 4.1 and 4.2 we have more general spectral multiplier results for the operators considered in the previous propositions.

\section{Asymptotically CONIC MANifolds}

Scattering manifolds or asymptotically conic manifolds are defined as the interior of a compact manifold with boundary $M$, and the metric $g$ is smooth on $M^{\circ}$ and has the form

$$
g=\frac{d x^{2}}{x^{4}}+\frac{h(x)}{x^{2}}
$$

in a collar neighbourhood near $\partial M$, where $x$ is a smooth boundary defining function for $M$ and $h(x)$ a smooth one-parameter family of metrics on $\partial M$; the function $r:=1 / x$ near $x=0$ can be 
thought of as a radial coordinate near infinity and the metric there is asymptotic to the exact metric cone $\left((0, \infty)_{r} \times \partial M, d r^{2}+r^{2} h(0)\right)$.

In this subsection we consider the following classical operators:

- Schrödinger operators, i.e. $-\Delta+V$ on $\mathbb{R}^{n}$, where $V$ smooth and decaying sufficiently at infinity;

- The Laplacian with respect to metric perturbations of the flat metric on $\mathbb{R}^{n}$, again decaying sufficiently at infinity;

- The Laplacian on asymptotically conic manifolds.

Proposition 9.1. Let $(M, g)$ be an asymptotically conic manifold of dimension $n \geq 3$, and let $x$ be a smooth boundary defining function of $\partial M$. Let $L:=-\Delta+V$ be a Schrödinger operator with $V \in x^{3} C^{\infty}(M)$ and assume that $L$ has no $L^{2}$-eigenvalues and that 0 is not a resonance. Then

(i) For any $\lambda_{0}>0$ there exists a constant $C>0$ such that the spectral measure $d E(\lambda)$ for $\sqrt{L}$ satisfies

$$
\left\|d E_{\sqrt{L}}(\lambda)\right\|_{L^{p}(M) \rightarrow L^{p^{\prime}}(M)} \leq C \lambda^{n\left(\frac{1}{p}-\frac{1}{p^{\prime}}\right)-1}
$$

for $1 \leq p \leq 2(n+1) /(n+3)$ and $0<\lambda \leq \lambda_{0}$.

(ii) If $(M, g)$ is nontrapping, then there exists $C>0$ such that (9.1) holds for all $\lambda>0$.

Proposition 9.1 was proved in [28, Theorem 1.2]. This proposition has useful consequence to establish the convergence of the Riesz means up to the critical exponent $\delta(p)=\max \{0, n \mid 1 / p-$ $1 / 2 \mid-1 / 2\}$ for all $1 \leq p \leq 2(n+1) /(n+3)$.

Corollary 9.2. Let $(M, g)$ be nontrapping and the operator L satisfies all assumptions of Proposition 9.1. Let $1 \leq p \leq 2(n+1) /(n+3)$. Then

(i) $S_{R}^{\delta(p)}(L)$ is of weak-type ( $\left.p, p\right)$ uniformly in $R$.

(ii) For any even bounded Borel function $F:[0, \infty) \rightarrow \mathbb{C}$ such that $\sup _{t>0}\left\|\eta \delta_{t} F\right\|_{W^{\beta, 2}}<\infty$ for some $\beta>\max \{n(1 / p-1 / 2), 1 / 2\}$ and some non-trivial function $\eta \in C_{c}^{\infty}(0, \infty)$, the operator $F(\sqrt{L})$ is bounded on $L^{r}(X)$ for all $p<r<p^{\prime}$ with

$$
\|F(\sqrt{L})\|_{r \rightarrow r} \leq C_{\beta}\left(\sup _{t>0}\left\|\eta \delta_{t} F\right\|_{W^{\beta, 2}}+|F(0)|\right) .
$$

Proof. This result is a straightforward application of Propositions 9.1, 2.4 and Theorems 5.1, 4.1.

\section{SCHRÖDINGER OPERATORS WITH ROUGH POTENTIALS}

This section is devoted to Shrödinger operators $-\Delta+V$ for which we prove new spectral multiplier results.

10.1. Schrödinger operators with inverse-square potential. We start with inverse square potentials, that is $V(x)=\frac{c}{|x|^{2}}$. Fix $n>2$ and assume that $-(n-2)^{2} / 4<c$. Define by quadratic form method $L=-\Delta+V$ on $L^{2}\left(\mathbb{R}^{n}, d x\right)$. The classical Hardy inequality

$$
-\Delta \geq \frac{(n-2)^{2}}{4}|x|^{-2}
$$

shows that for all $c>-(n-2)^{2} / 4$, the self-adjoint operator $L$ is non-negative. Set $p_{c}^{*}=n / \sigma$, $\sigma=\max \left\{(n-2) / 2-\sqrt{(n-2)^{2} / 4+c}, 0\right\}$. If $c \geq 0$ then the semigroup $\exp (-t L)$ is pointwise 
bounded by the Gaussian semigroup and hence act on all $L^{p}$ spaces with $1 \leq p \leq \infty$. If $c<0$, then $\exp (-t L)$ acts as a uniformly bounded semigroup on $L^{p}\left(\mathbb{R}^{n}\right)$ for $p \in\left(\left(p_{c}^{*}\right)^{\prime}, p_{c}^{*}\right)$ and the range $\left(\left(p_{c}^{*}\right)^{\prime}, p_{c}^{*}\right)$ is optimal (see for example [41]).

It is proved in [10] that the solution $u(t)=e^{-i t L} f$ of the corresponding Schrödinger equation

$$
i \partial_{t} u+L u=0, u(0)=f
$$

satisfies Strichatrz estimate (6.2). The smoothing property (7.6) is proved in [4]. Therefore, we obtain from Proposition 7.3 that $L$ satisfies restriction estimate $\left(\mathrm{R}_{\mathrm{p}}\right)$ for all $p \in\left(\left(p_{c}^{*}\right)^{\prime}, \frac{2 n}{n+2}\right]$. If $c \geq 0$, then $p=\left(p_{c}^{*}\right)^{\prime}=1$ is included. From this and Theorems 5.1 and 7.4 we obtain

Theorem 10.1. Suppose that $n>2$ and $-(n-2)^{2} / 4<c$ and that $p \in\left(\left(p_{c}^{*}\right)^{\prime}, 2 n /(n+2)\right]$ where $p_{c}^{*}=n / \sigma$ and $\sigma=\max \left\{(n-2) / 2-\sqrt{(n-2)^{2} / 4+c}, 0\right\}$ and $\left(p_{c}^{*}\right)^{\prime}$ its dual exponent. Then

(i) $S_{R}^{\delta(p)}(L)$ is of weak-type ( $\left.p, p\right)$ uniformly in $R$.

(ii) For any even bounded Borel function $F:[0, \infty) \rightarrow \mathbb{C}$ such that $\sup _{t>0}\left\|\eta \delta_{t} F\right\|_{W^{\beta, 2}}<\infty$ for some $\beta>\max \{n(1 / p-1 / 2), 1 / 2\}$ and some non-trivial function $\eta \in C_{c}^{\infty}(0, \infty)$, the operator $F(\sqrt{L})$ is bounded on $L^{r}(X)$ for all $p<r<p^{\prime}$ with

$$
\|F(\sqrt{L})\|_{r \rightarrow r} \leq C_{\beta}\left(\sup _{t>0}\left\|\eta \delta_{t} F\right\|_{W^{\beta, 2}}+|F(0)|\right) .
$$

10.2. Scattering operators. Assume now that $n=3$ and $V$ is a real-valued measurable function such that

$$
\int_{\mathbb{R}^{6}} \frac{|V(x)||V(y)|}{|x-y|^{2}} d x d y<(4 \pi)^{2} \quad \text { and } \quad \sup _{x \in \mathbb{R}^{3}} \int_{\mathbb{R}^{3}} \frac{|V(y)|}{|x-y|} d y<4 \pi .
$$

The following proposition is a consequence of Proposition 7.1 and the main result in Rodnianski and Schlag [49] which gives the dispersive estimate for $\exp (i t(-\Delta+V))$ on $\mathbb{R}^{3}$.

Proposition 10.2. Suppose that $L=-\Delta+V$ on $\mathbb{R}^{3}$ with a real-valued $V$ which satisfies (10.2). Then L satisfies $\left(\mathrm{R}_{\mathrm{p}}\right)$ for all $1 \leq p<6 / 5$.

In the special case $p=1$, Proposition 10.2 was obtain in [23, Theorem 7.15] for compactly supported function $V \geq 0$ which satisfies (10.2). The following result is a consequence of Theorem 5.1, 4.1 and Proposition 10.2.

Corollary 10.3. Suppose that $L=-\Delta+V$ on $\mathbb{R}^{3}$ and that $V$ satisfies assumption of Proposition 10.2. Assume also that $1 \leq p<6 / 5$. Then

(i) $S_{R}^{\delta(p)}(L)$ is of weak-type ( $\left.p, p\right)$ uniformly in $R$.

(ii) For any even bounded Borel function $F:[0, \infty) \rightarrow \mathbb{C}$ such that $\sup _{t>0}\left\|\eta \delta_{t} F\right\|_{W^{\beta, 2}}<\infty$ for some $\beta>\max \{3(1 / p-1 / 2), 1 / 2\}$ and some non-trivial function $\eta \in C_{c}^{\infty}(0, \infty)$, the operator $F(\sqrt{L})$ is bounded on $L^{r}(X)$ for all $p<r<p^{\prime}$.

If $n \geq 3$ and potential $V \in W^{s, 2}\left(\mathbb{R}^{n}\right)$ for some $s>\frac{n}{2}-1$ and has fast decay, Bourgain [8] proved the dispersive estimate for $\exp (i t(-\Delta+V))$. Our results apply for $L=-\Delta+V$ and allow to obtain sharp spectral multiplier results. We also refer to Rodnianski and Schlag [49] for more references on dispersive estimates for Schrödinger operators.

We also mention that Strichartz estimates are proved for a class of elliptic operators with variable coefficients by J. Marzuola, J. Metcalfe and D. Tataru [43] (see Theorem 1.20). Therefore, the same reasoning as for the Theorem 10.1 allows us to obtain sharp spectral multipliers and endpoint Bochner-Riesz summability for these elliptic operators. 
10.3. The harmonic oscillator. In this section we focus on Schrödinger operators such as the harmonic oscillator $-\Delta+|x|^{2}$ on $L^{2}\left(\mathbb{R}^{n}\right)$ for $n \geq 2$. As in [39] we can also consider Schrödinger operators $L=-\Delta+V$ with a positive potential $V$ which satisfies the following condition

$$
V \sim|x|^{2}, \quad|\nabla V| \sim|x|, \quad\left|\partial_{x}^{2} V\right| \leq 1 .
$$

We apply Theorems 3.6 and 4.2 and the results from [39] to prove sharp results on Bochner-Riesz summability and singular spectral multipliers for $L$. Bochner-Riesz summability results for the harmonic oscillator were obtained first by Kardzhov [36]. Here we describe an alternative proof. The corresponding singular integral multiplier is a new result. The following theorem is the main goal of this section.

Theorem 10.4. Assume that potential $V$ satisfies condition (10.3) and set $L=-\Delta+V$. Let $1 \leq p \leq 2 n /(n+2)$. Then

(i) For any even function $F$ such that $\operatorname{supp} F \subseteq[-1,1]$ and $\|F\|_{W^{\beta, 2}}<\infty$ for some $\beta>$ $\max \{n(1 / p-1 / 2), 1 / 2\}$, the operator $F(t \sqrt{L})$ is bounded on $L^{p}(X)$ for all $t>0$ and

$$
\sup _{t>0}\|F(t \sqrt{L})\|_{p \rightarrow p} \leq C\|F\|_{W^{\beta, 2}} .
$$

(ii) For any even bounded Borel function $F:[0, \infty) \rightarrow \mathbb{C}$ such that $\sup _{t>0}\left\|\eta \delta_{t} F\right\|_{W^{\beta, 2}}<\infty$ for some $\beta>\max \{n(1 / p-1 / 2), 1 / 2\}$ and some non-trivial function $\eta \in C_{c}^{\infty}(0, \infty)$, the operator $F(\sqrt{L})$ is bounded on $L^{r}(X)$ for all $p<r<p^{\prime}$. In addition,

$$
\|F(\sqrt{L})\|_{r \rightarrow r} \leq C_{\beta}\left(\sup _{t>0}\left\|\eta \delta_{t} F\right\|_{W^{\beta, 2}}+|F(0)|\right) .
$$

Proof. It follows from Theorem 4 in [39] that for all $\lambda \geq 0$ and all $1 \leq p \leq 2 n /(n+2)$

$$
\left\|E_{L}\left[\lambda^{2}, \lambda^{2}+1\right)\right\|_{p \rightarrow 2} \leq C(1+\lambda)^{n\left(\frac{1}{p}-\frac{1}{2}\right)-1} .
$$

Take a function $F$ with support in $[-N, N]$. We have as in the proof of Proposition 3.10

$$
\begin{aligned}
\|F(\sqrt{L})\|_{p \rightarrow 2}^{2} & \leq \sum_{\ell=1}^{N^{2}}\left\|E_{\sqrt{L}}\left[\frac{\ell-1}{N}, \frac{\ell}{N}\right) F(\sqrt{L})\right\|_{p \rightarrow 2}^{2} \\
& \leq \sum_{\ell=1}^{N^{2}} \sup _{\lambda \in\left[\frac{\ell-1}{N}, \frac{\ell}{N}\right)}|F(\lambda)|^{2}\left\|E_{\sqrt{L}}\left[\frac{\ell-1}{N}, \frac{\ell}{N}\right)\right\|_{p \rightarrow 2}^{2} \\
& =\sum_{\ell=1}^{N^{2}} \sup _{\lambda \in\left[\frac{\ell-1}{N}, \frac{\ell}{N}\right)}|F(\lambda)|^{2}\left\|E_{L}\left[\left(\frac{\ell-1}{N}\right)^{2},\left(\frac{\ell}{N}\right)^{2}\right)\right\|_{p \rightarrow 2}^{2} .
\end{aligned}
$$

Now we observe that for all $\ell=1,2, \cdots, N^{2}$

$$
\left\|E_{L}\left[\left(\frac{\ell-1}{N}\right)^{2},\left(\frac{\ell}{N}\right)^{2}\right)\right\|_{p \rightarrow 2}^{2} \leq\left\|E_{L}\left[\left(\frac{\ell-1}{N}\right)^{2},\left(\frac{\ell-1}{N}\right)^{2}+2\right)\right\|_{p \rightarrow 2}^{2} .
$$

This, in combination with (10.4) and (10.5), shows that for $1 \leq p \leq 2 n /(n+2)$

$$
\begin{aligned}
\|F(\sqrt{L})\|_{p \rightarrow 2}^{2} & \leq \sum_{\ell=1}^{N^{2}} \sup _{\lambda \in\left[\frac{\ell-1}{N}, \frac{\ell}{N}\right)}|F(\lambda)|^{2}\left(2+\frac{\ell-1}{N}\right)^{2 n\left(\frac{1}{p}-\frac{1}{2}\right)-2} \\
& \leq C N^{2 n\left(\frac{1}{p}-\frac{1}{2}\right)} \frac{1}{N^{2}} \sum_{\ell=1}^{N^{2}} \sup _{\lambda \in\left[\frac{\ell-1}{N}, \frac{\ell}{N}\right)}|F(\lambda)|^{2}
\end{aligned}
$$




$$
\leq C N^{2 n\left(\frac{1}{p}-\frac{1}{2}\right)} \frac{1}{N^{2}} \sum_{\ell=1}^{N^{2}} \sup _{\lambda \in\left[\frac{\ell-1}{N^{2}}, \frac{\ell}{N^{2}}\right)}|F(N \lambda)|^{2} .
$$

This proves $\left(\mathrm{SC}_{\mathrm{p}, 2}^{2, \kappa}\right)$ for $\kappa=2$ and $p$ such that $1 \leq p \leq 2 n /(n+2)$. It remains to show $\left(\mathrm{AB}_{\mathrm{p}}\right)$ and then apply Theorems 3.6 and 4.2. Now condition $\left(\mathrm{AB}_{\mathrm{p}}\right)$ follows from the following lemma.

Lemma 10.5. Let $L=-\Delta+V$, where $V \in L_{\mathrm{loc}}^{1}\left(\mathbb{R}^{n}\right)$ and $V \geq 0$. Suppose that for some $\kappa>0$ and any $\epsilon>0$

$$
\int_{\mathbb{R}^{n}}(1+V(x))^{n(1-\kappa) / 2-\epsilon} d x<\infty .
$$

Then condition $\left(\mathrm{SC}_{\mathrm{p}, 2}^{2, \kappa}\right)$ implies $\left(\mathrm{AB}_{\mathrm{p}}\right)$.

The proof of Lemma 10.5 is a straightforward modification of the proof of Lemma 7.9 of [23] so we skip it here.

\section{Operators $\Delta_{n}+\frac{c}{r^{2}}$ ACTING ON $L^{2}\left((0, \infty), r^{n-1} d r\right)$}

In this section we consider a class of Schrödinger operators on $L^{2}\left((0, \infty), r^{n-1} d r\right)$. These operators generate semigroups but do not have the classical Gaussian upper bound for the heat kernel.

Fix $n>2$ and $c>-(n-2)^{2} / 4$ and consider the space $L^{2}\left((0, \infty), r^{n-1} d r\right)$. For $f, g \in C_{c}^{\infty}(0, \infty)$ we define the quadratic form

$$
Q_{n, c}^{(0, \infty)}(f, g)=\int_{0}^{\infty} f^{\prime}(r) g^{\prime}(r) r^{n-1} d r+\int_{0}^{\infty} \frac{c}{r^{2}} f(r) g(r) r^{n-1} d r .
$$

Using the Friedrichs extension one can define the operator $L_{n, c}=\Delta_{n}+c / r^{2}$ as the unique selfadjoint operator corresponding to $Q_{n, c}^{(0, \infty)}$, acting on $L^{2}\left((0, \infty), r^{n-1} d r\right)$. In the sequel we will write $L$ instead of $L_{n, c}$, which is formally given by the following formula

$$
L f=\left(\Delta_{n}+\frac{c}{r^{2}}\right) f=-\frac{d^{2}}{d r^{2}} f-\frac{n-1}{r} \frac{d}{d r} f+\frac{c}{r^{2}} f .
$$

The classical Hardy inequality (10.1) shows that for all $c>-(n-2)^{2} / 4$, the self-adjoint operator $L$ is non-negative. Such operators can be seen as radial Schrödinger operators with inverse-square potentials. It follows by Theorem 3.3 of [19] that $L$ satisfies Davies-Gaffney estimate, which in turns implies property (FS).

Now for $-(n-2)^{2} / 4<c<0$, we set $p_{c}^{*}=n / \sigma$ where $\sigma=(n-2) / 2-\sqrt{(n-2)^{2} / 4+c}$ and $\left(p_{c}^{*}\right)^{\prime}$ its dual exponent. Note that $2<\frac{2 n}{n-2}<p_{c}^{*}$. Liskevich, Sobol and Vogt [41] proved that for all $t>0$ and all $p \in\left(\left(p_{c}^{*}\right)^{\prime}, p_{c}^{*}\right)$,

$$
\left\|e^{-t L}\right\|_{p \rightarrow p} \leq C
$$

They also proved that range $\left(\left(p_{c}^{*}\right)^{\prime}, p_{c}^{*}\right)$ is optimal and that for all $p \notin\left(\left(p_{c}^{*}\right)^{\prime}, p_{c}^{*}\right)$, the semigroup does not even act on $L^{p}\left((0, \infty), r^{n-1} d r\right)$ (see also [22, 19, 29]).

Proposition 11.1. Suppose that $n>2$ and $-(n-2)^{2} / 4<c$. For $c<0$, set $p \in\left(\left(p_{c}^{*}\right)^{\prime}, \frac{2 n}{n+1}\right)$ where $p_{c}^{*}=n / \sigma$ and $\sigma=(n-2) / 2-\sqrt{(n-2)^{2} / 4+c}$ and $\left(p_{c}^{*}\right)^{\prime}$ its conjugate exponent. For $c \geq 0$, set $p \in\left[1, \frac{2 n}{n+1}\right)$. Then for any $R>0$ and all Borel functions $F$ such that $\operatorname{supp} F \subset[0, R]$,

$$
\left\|F(\sqrt{L}) P_{B\left(x_{B}, r_{B}\right)}\right\|_{p \rightarrow 2} \leq C V\left(x_{B}, r_{B}\right)^{\frac{1}{2}-\frac{1}{p}}\left(R r_{B}\right)^{n\left(\frac{1}{p}-\frac{1}{2}\right)}\left\|\delta_{R} F\right\|_{2}
$$


for all $x_{B} \in \mathbb{R}_{+}$and $r_{B} \geq 1 / R$.

Proof. In [29] the explicit formula for the resolvent of the operator $L=\Delta_{n}+\frac{c}{r^{2}}$ is described. Based on this formula we calculate explicitly the spectral projections $d E_{\sqrt{L}}(\lambda)$ (This calculation was shown to us by Andrew Hassell). Define the number $n^{\prime}=n^{\prime}(n, c)$ to be the maximum positive root of the equation $\left(n^{\prime} / 2-1\right)^{2}=(n / 2-1)^{2}+c$ so that $p_{c}^{*}=2 n /\left(n-n^{\prime}\right)$. Hence, $p_{c}^{*}=n / \sigma$ where $\sigma=(n-2) / 2-\sqrt{(n-2)^{2} / 4+c}$. Next let $K_{\alpha}$ and $I_{\alpha}$ are modified Bessel function, see [1, $\$ 9.6 .1 \mathrm{p}$. 374] or [66, §1.14 p. 16]. Set $\ell(x)=x^{-n / 2+1} I_{n^{\prime} / 2-1}(x)$ and $k(x)=x^{-n / 2+1} K_{n^{\prime} / 2-1}(x)$. Then by (4.2) and Section 6.1 of [29] the resolvent kernel for $L=\Delta_{n}+\frac{c}{r^{2}}$ is given by

$$
R(\lambda)(x, y)=K_{\left(L+\lambda^{2}\right)^{-1}}(x, y)= \begin{cases}v \lambda^{d-2} k(\lambda y) \ell(\lambda x) & \text { if } y \geq x, \\ v \lambda^{d-2} \ell(\lambda y) k(\lambda x) & \text { if } x>y\end{cases}
$$

for some constant $v$. Next recall that $x^{-\alpha} I_{\alpha}$ is an even analytic function and that

$$
K_{\alpha}(x)=\frac{\pi}{2} \frac{I_{-\alpha}(x)-I_{\alpha}(x)}{\sin (\alpha \pi)},
$$

see $[1, \S 9.6 .1$ p. 374]. Hence by the limiting absorption principle (that is Stone's Theorem) for all $x \leq y$

$$
\begin{aligned}
K_{d E_{\sqrt{L}}(\lambda)}(x, y)= & \frac{i \lambda}{\pi}(R(i \lambda)(x, y)-R(-i \lambda)(x, y)) \\
= & v \frac{i \lambda}{\pi} x^{1-n / 2} y^{1-n / 2} I_{n^{\prime} / 2-1}(i \lambda x) \frac{I_{1-n^{\prime} / 2}(i \lambda y)-I_{n^{\prime} / 2-1}(i \lambda y)}{\sin \left(\left(n^{\prime} / 2-1\right) \pi\right)} \\
& -v \frac{i \lambda}{\pi} x^{1-n / 2} y^{1-n / 2} I_{n^{\prime} / 2-1}(-i \lambda x) \frac{I_{1-n^{\prime} / 2}(-i \lambda y)-I_{n^{\prime} / 2-1}(-i \lambda y)}{\sin \left(\left(n^{\prime} / 2-1\right) \pi\right)} .
\end{aligned}
$$

Recall next that $x^{-\alpha} I_{\alpha}$ is an even analytic function so

$$
I_{n^{\prime} / 2-1}(i \lambda x) I_{1-n^{\prime} / 2}(i \lambda y)=I_{n^{\prime} / 2-1}(-i \lambda x) I_{1-n^{\prime} / 2}(-i \lambda y)
$$

and

$$
I_{n^{\prime} / 2-1}(-i \lambda x) I_{n^{\prime} / 2-1}(-i \lambda y)=e^{i \pi\left(n^{\prime}-2\right)} I_{n^{\prime} / 2-1}(i \lambda x) I_{n^{\prime} / 2-1}(i \lambda y) .
$$

Thus

$$
\begin{aligned}
K_{d E_{\sqrt{L}}(\lambda)}(x, y) & =v \frac{i \lambda}{\pi} x^{1-n / 2} y^{1-n / 2}\left(e^{i \pi\left(n^{\prime}-2\right)}-1\right) \frac{I_{n^{\prime} / 2-1}(i \lambda x) I_{n^{\prime} / 2-1}(i \lambda y)}{\sin \left(\left(n^{\prime} / 2-1\right) \pi\right)} \\
& =\frac{2 i v}{\pi} e^{i \pi\left(n^{\prime} / 2-1\right)} \lambda x^{1-n / 2} y^{1-n / 2} I_{n^{\prime} / 2-1}(i \lambda x) I_{n^{\prime} / 2-1}(i \lambda y) .
\end{aligned}
$$

We prove equality (11.4) under assumption that $x \leq y$ but similar argument shows that (11.4) holds for all $x$ and $y$. Now if we set $\ell(x)=x^{-n / 2+1} I_{n^{\prime} / 2-1}(i x)$ then by (11.4)

$$
d E_{\sqrt{L}}(\lambda) f(x)=C \lambda^{n-1} \ell(\lambda x) \int_{0}^{\infty} \ell(\lambda y) f(y) y^{n-1} d y
$$

for some constant $C$. The function $\ell=\ell_{n, c}(\lambda)$ for $n>2$ satisfies the following estimates $([1,66])$ :

By (11.5)

$$
|\ell(\lambda)| \leq\left\{\begin{array}{lll}
\lambda^{\frac{n^{\prime}-n}{2}} & \text { if } & \lambda \leq 1 \\
\lambda^{\frac{1-n}{2}} & \text { if } & 1 \leq \lambda
\end{array}\right.
$$

$$
K_{F(\sqrt{L})}(x, y)=C \int_{0}^{\infty} F(\lambda) \ell(\lambda x) \ell(\lambda y) \lambda^{n-1} d \lambda
$$


Let us prove our estimate (11.2). We consider only the case $-(n-2)^{2} / 4<c<0$. The proof is similar for the case $c \geq 0$. For every $B=B\left(x_{B}, r_{B}\right)$, one writes

Hence

$$
F(\sqrt{L}) P_{B} f(x)=C \int_{0}^{\infty}\left(\int_{0}^{\infty} F(\lambda) \ell(\lambda x) \ell(\lambda y) \lambda^{n-1} d \lambda\right) \chi_{B}(y) f(y) y^{n-1} d y
$$

$$
\begin{aligned}
\left\|F(\sqrt{L}) P_{B} f\right\|_{2}^{2} & =C \int_{0}^{\infty}\left|\int_{0}^{\infty}\left(\int_{0}^{\infty} F(\lambda) \ell(\lambda x) \ell(\lambda y) \lambda^{n-1} d \lambda\right) \chi_{B}(y) f(y) y^{n-1} d y\right|^{2} x^{n-1} d x \\
& =C \int_{0}^{\infty}\left|\int_{0}^{\infty} \ell(\lambda x) \lambda^{n-1} \int_{0}^{\infty} F(\lambda) \ell(\lambda y) \chi_{B}(y) f(y) y^{n-1} d y d \lambda\right|^{2} x^{n-1} d x .
\end{aligned}
$$

Note that the following Plancherel type equality is satisfied

$$
\int_{0}^{\infty}\left|\int_{0}^{\infty} F(\lambda) \ell(\lambda x) \lambda^{n-1} d \lambda\right|^{2} x^{n-1} d x=\int_{0}^{\infty}|F(\lambda)|^{2} \lambda^{n-1} d \lambda
$$

which yields

$$
\begin{aligned}
\left\|F(\sqrt{L}) P_{B} f\right\|_{2}^{2} & =C \int_{0}^{\infty}|F(\lambda)|^{2}\left|\int_{0}^{\infty} \ell(\lambda y) \chi_{B}(y) f(y) y^{n-1} d y\right|^{2} \lambda^{n-1} d \lambda \\
& \leq C \int_{0}^{\infty}|F(\lambda)|^{2}\left\|\ell(\lambda y) \chi_{B}(y)\right\|_{p^{\prime}}^{2}\|f\|_{p}^{2} \lambda^{n-1} d \lambda .
\end{aligned}
$$

Case I: $B=B\left(x_{B}, r_{B}\right)$ and $x_{B} \leq 2 r_{B}$. Hence

$$
\begin{aligned}
\left\|\ell(\lambda y) \chi_{B}\right\|_{p^{\prime}}^{p^{\prime}} & \leq C \int_{0}^{2 r_{B}}|\ell(\lambda y)|^{p^{\prime}} y^{n-1} d y \\
& \leq C \int_{0}^{\infty}|\ell(\lambda y)|^{p^{\prime}} y^{n-1} d y \\
& \leq C \lambda^{-n} \int_{0}^{\infty}|\ell(y)|^{p^{\prime}} y^{n-1} d y \\
& \leq C \lambda^{-n}
\end{aligned}
$$

for all $2 n /(n-1)<p^{\prime}<p_{c}^{*}=2 n /\left(n-n^{\prime}\right)$ (For $c \geq 0$ this condition should be replaced by $\left.2 n /(n-1)<p^{\prime}\right)$. Thus

$$
\begin{aligned}
\left\|F(\sqrt{L}) P_{B} f\right\|_{2}^{2} & \leq C \int_{0}^{\infty}|F(\lambda)|^{2} \lambda^{n-1-\frac{2 n}{p^{\prime}}} d \lambda\|f\|_{p}^{2} \\
& \leq C R^{n-\frac{2 n}{p^{\prime}}}\left\|\delta_{R} F\right\|_{2}^{2}\|f\|_{p}^{2} .
\end{aligned}
$$

When $x_{B} \leq 2 r_{B}$, we have that $V(B) \approx r_{B}^{n}$ and $V(B)^{1 / 2-1 / p} r_{B}^{n(1 / p-1 / 2)} \approx 1$. This gives

$$
\begin{aligned}
\left\|F(\sqrt{L}) \chi_{B} f\right\|_{2}^{2} & \leq C V(B)^{2\left(\frac{1}{2}-\frac{1}{p}\right)} r_{B}^{2 n\left(\frac{1}{p}-\frac{1}{2}\right)} R^{n-\frac{2 n}{p^{\prime}}}\left\|\delta_{R} F\right\|_{2}^{2}\|f\|_{p}^{2} \\
& \leq C V(B)^{2\left(\frac{1}{2}-\frac{1}{p}\right)}\left(R r_{B}\right)^{2 n\left(\frac{1}{p}-\frac{1}{2}\right)}\left\|\delta_{R} F\right\|_{2}^{2}\|f\|_{p}^{2} .
\end{aligned}
$$

This proves Case I.

Case II: $B=B\left(x_{B}, r_{B}\right)$ and $x_{B}>2 r_{B}$. Then

$$
\left\|\ell(\lambda y) \chi_{B}\right\|_{p^{\prime}}^{p^{\prime}} \leq \int_{x_{B}-r_{B}}^{x_{B}+r_{B}}|\ell(\lambda y)|^{p^{\prime}} y^{n-1} d y
$$




$$
\begin{aligned}
& \leq C \int_{x_{B}-r_{B}}^{x_{B}+r_{B}}(\lambda y)^{\frac{(1-n) p^{\prime}}{2}} y^{n-1} d y \\
& \leq C \lambda^{p^{\prime} \frac{1-n}{2}} r_{B} x_{B}^{n-1+p^{\prime} \frac{1-n}{2}} .
\end{aligned}
$$

Hence

$$
\begin{aligned}
\left\|F(\sqrt{L}) P_{B} f\right\|_{2}^{2} & \leq C \int_{0}^{\infty}|F(\lambda)|^{2} r_{B}^{\frac{2}{p^{\prime}}} x_{B}^{2\left(n-1+\frac{(1-n) p^{\prime}}{2}\right) / p^{\prime}} d \lambda\|f\|_{p}^{2} \\
& \leq C r_{B}^{\frac{2}{p^{\prime}}} x_{B}^{\frac{2(n-1)}{p^{\prime}}-n+1} R\left\|\delta_{R} F\right\|_{2}^{2}\|f\|_{p}^{2} .
\end{aligned}
$$

Note that if $x_{B}>2 r_{B}$ then $V(B) \approx x_{B}^{n-1} r_{B}$. Thus

$$
\begin{aligned}
\left\|F(\sqrt{L}) P_{B} f\right\|_{2}^{2} & \leq C r_{B}^{\frac{2}{p^{\prime}}} x_{B}^{\frac{2(n-1)}{p^{\prime}}-n+1} R\left\|\delta_{R} F\right\|_{2}^{2}\|f\|_{p}^{2} \\
& \leq C V(B)^{2\left(\frac{1}{2}-\frac{1}{p}\right)}\left(R r_{B}\right)^{2 n\left(\frac{1}{p}-\frac{1}{2}\right)}\left(R r_{B}\right)^{1-2 n\left(\frac{1}{p}-\frac{1}{2}\right)}\left\|\delta_{R} F\right\|_{2}^{2}\|f\|_{p}^{2} \\
& \leq C V(B)^{2\left(\frac{1}{2}-\frac{1}{p}\right)}\left(R r_{B}\right)^{2 n\left(\frac{1}{p}-\frac{1}{2}\right)}\left\|\delta_{R} F\right\|_{2}^{2}\|f\|_{p}^{2}
\end{aligned}
$$

according to the condition $\operatorname{Rr}_{B} \geq 1$ and $p^{\prime}>2 n /(n-1)$. This proves Case II, and then the proof of Proposition 11.1 is complete.

Corollary 11.2. Suppose that $n>2$ and $-(n-2)^{2} / 4<c$. For $c<0$, set $p \in\left(\left(p_{c}^{*}\right)^{\prime}, \frac{2 n}{n+1}\right)$ where $p_{c}^{*}=n / \sigma$ and $\sigma=(n-2) / 2-\sqrt{(n-2)^{2} / 4+c}$ and $\left(p_{c}^{*}\right)^{\prime}$ its conjugate exponent. For $c \geq 0$, set $p \in\left[1, \frac{2 n}{n+1}\right)$. Then

(i) $S_{R}^{\delta(p)}(L)$ is of weak-type ( $\left.p, p\right)$ uniformly in $R$.

(ii) For any bounded Borel function $F:[0, \infty) \rightarrow \mathbb{C}$ such that $\sup _{t>0}\left\|\eta \delta_{t} F\right\|_{W^{\beta, 2}}<\infty$ for some $\beta>\max \{n(1 / p-1 / 2), 1 / 2\}$ and some non-trivial function $\eta \in C_{c}^{\infty}(0, \infty)$, the operator $F(\sqrt{L})$ is bounded on $L^{r}(X)$ for all $p<r<p^{\prime}$ with

$$
\|F(\sqrt{L})\|_{r \rightarrow r} \leq C_{\beta}\left(\sup _{t>0}\left\|\eta \delta_{t} F\right\|_{W^{\beta, 2}}+|F(0)|\right) .
$$

Proof. This result is straightforward from Proposition 11.1 and Theorems 5.1, 4.1.

Remark 11.3. Note that for the standard Laplacian $\Delta$ on $\mathbb{R}^{n}$ Stein-Tomas estimate $\left(\mathrm{R}_{\mathrm{p}}\right)$ holds if and only if $1 \leq p \leq 2(n+1) /(n+3)$. Surprisingly, if $n>2$ and $-(n-2)^{2} / 4<c<0$, then the restriction estimate $\left(\mathrm{ST}_{\mathrm{p}, 2}^{2}\right)$ for the operator $\Delta_{n}+\frac{c}{r^{2}}$ holds only for all $p \in\left(\left(p_{c}^{*}\right)^{\prime}, 2 n /(n+1)\right)$ where $p_{c}^{*}=n / \sigma$ and $\sigma=(n-2) / 2-\sqrt{(n-2)^{2} / 4+c}$.

\section{EXAMPLES OF $(1,2)$-RESTRICTION TYPE CONDITIONS}

In [23] the following Plancharel condition is introduced: for any $R>0$ and all even Borel functions $F$ such that $\operatorname{supp} F \subseteq[0, R]$,

$$
\int_{X}\left|K_{F(\sqrt{L})}(x, y)\right|^{2} d \mu(x) \leq C V\left(y, R^{-1}\right)^{-1}\left\|\delta_{R} F\right\|_{q}^{2}
$$

for some $q \in[2, \infty]$. Here $m \geq 2$ denotes the order of operator $L$ and for operators which we consider here $m=2($ see $[23,(3.1)])$. Note that for every $x \in X$ and $r \geq 1 / R$,

$$
\left\|F(\sqrt{L}) P_{B(x, r)} f\right\|_{2} \leq\left\|\int_{X} K_{F(\sqrt{L})}(z, w) \chi_{B(x, r)}(w) f(w) d \mu(w)\right\|_{2}
$$




$$
\begin{aligned}
& \leq \int_{X}\left\|K_{F(\sqrt{L})}(\cdot, w)\right\|_{2} \chi_{B(x, r)}(w)|f(w)| d \mu(w) \\
& \leq C\left\|\delta_{R} F\right\|_{q} \int_{X} V\left(w, R^{-1}\right)^{-1 / 2} \chi_{B(x, r)}(w)|f(w)| d \mu(w) \\
& \leq C V(x, r)^{-1 / 2}(R r)^{n / 2}\left\|\delta_{R} F\right\|_{q}\|f\|_{1},
\end{aligned}
$$

where in the last inequality we used the doubling condition (2.2). Therefore, for every $x \in X$ and $r \geq 1 / R$

$$
\left\|F(\sqrt{L}) P_{B(x, r)}\right\|_{1 \rightarrow 2} \leq C V(x, r)^{-1 / 2}(R r)^{n / 2}\left\|\delta_{R} F\right\|_{q},
$$

and so the condition (12.1) is just a slightly stronger version of condition $\left(\mathrm{ST}_{1,2}^{\mathrm{q}}\right)$.

It was noted in [23] that condition (12.1) with $q=2$ holds for homogeneous sub-Laplacian acting on homogeneous Lie groups. It is shown in [54] that (12.1) with $q=2$ holds also for "quasi-homogeneous" subelliptic and elliptic operators. As we note condition (12.1) is stronger than condition $\left(\mathrm{ST}_{1,2}^{2}\right)$ so this implies the following result.

Proposition 12.1. Let $L$ be a homogeneous sub-Laplacian or "quasi-homogeneous" operator acting on homogeneous Lie group with homogeneous dimension d. Then the Riesz mean $S_{R}^{(d-1) / 2}(L)$ of order $(d-1) / 2$ is of weak-type $(1,1)$ uniformly in $R$.

Proof. Proposition 12.1 follows directly from Theorem 5.1.

We believe that in this generality Proposition 12.1 is a new result. However in the case of Heisenberg group it follows from the result obtained by Müller, Stein and Hebisch that the the Riesz means of order $\delta>\left(d_{e}-1\right) / 2$ is bounded on $L^{1}$, where $d_{e}<d$ is the topological dimension of the Heisenberg(see [47, 30]). Therefore it is likely that Proposition 12.1 is not a genuine endpoint result.

Acknowledgements: A. Sikora was partly supported by Australian Research Council Discovery Grant DP 110102488. L. Yan was supported by NNSF of China (Grant No. 10925106), Guangdong Province Key Laboratory of Computational Science and the Fundamental Research Funds for the Central Universities (Grant No. 09lgzs610). Part of this work was done while E.M. Ouhabaz was visiting Macquarie University. His visit was partly supported by ARC Discovery Grant DP 110102488 and CNRS. He wishes to thank X.T. Duong for the invitation. A. Sikora and E.M. Ouhabaz would like to thank M. Cowling, X.T. Duong, A. Hassell and A. McIntosh for fruitful discussions.

\section{REFERENCES}

[1] M. Abramowitz and I.A. Stegun, Handbook of mathematical functions with formulas, graphs, and mathematical tables. National Bureau of Standards Applied Mathematics Series, 55, U.S. Government Printing Office, Washington, D.C. 1964. 44

[2] G. Alexopoulos, Spectral multipliers on Lie groups of polynomial growth. Proc. Amer. Math. Soc. 120 (1994), no. 3, 973-979. 2

[3] L. Ambrosio, M. Miranda Jr and D. Pallara, Special functions of bounded variation in doubling metric measure spaces, Calculus of variations: topics from the mathematical heritage of E. De Giorgi, Quad. Mat., Dept. Math., Seconda Univ. Napoli, Caserta, 14 (2004), 1-45. 7, 13

[4] J. Assaad, Riesz transforms associated to Schrödinger operators with negative potentials. Publ. Mat. 55 (2011), no. 1, 123-150. 41

[5] J. Bergh and J. Löfström, Interpolation spaces. Springer-Verlag, Berlin- New York, 1976. 15, 27

[6] S. Blunck, A Hörmander-type spectral multiplier theorem for operators without heat kernel. Ann. Sc. Norm. Super. Pisa Cl. Sci. (5), 2 (2003), no. 3, 449-459. 20 
[7] S. Blunck and P.C. Kunstmann, Calderón-Zygmund theory for non-integral operators and the $H^{\infty}$ functional calculus. Rev. Mat. Iberoamericana, 19 (2003), no. 3, 919-942. 20

[8] J. Bourgain, On long-time behaviour of solutions of linear Schrödinger equations with smooth timedependent potentials. Geometric Aspects of Functional Analysis, 99-113, Lecture Notes in Math. 1807, Springer, Berlin, 2003. 41

[9] J. Bourgain and L. Guth, Bounds on oscillatory integral operators based on multilinear estimates. Geom. Funct. Anal. to appear (2011), available at http://arXiv.org/abs/1012.3760 3

[10] N. Burq, F. Planchon, J.G. Stalker and A. Tahvildar-Zadeh, Strichartz estimates for the wave and Schrödinger equations with the inverse-square potential. J. Funct. Anal. 203 (2003), no. 2, 519-549. 7, 41

[11] A. Carbery, Variants of the Calderón-Zygmund theory for $L^{p}$-spaces. Rev. Mat. Iberoamericana, 2 (1986), no. 4, 381-396. 4, 19, 21, 22

[12] L. Carleson and P. Sjölin, Oscillatory integrals and a multiplier problem for the disc. Studia Math. 44 (1972), 287-299. 2, 3

[13] J. Cheeger, M. Gromov and M. Taylor, Finite propagation speed, kernel estimates for functions of the Laplace operator, and the geometry of complete Riemannian manifolds. J. Differential Geom. 17 (1982), no. $1,15-53.8$

[14] M. Christ, Weak type endpoint bounds for Bochner-Riesz multipliers. Rev. Mat. Iberoamericana, 3 (1987), no. $1,25-31.2,5,6,27,39$

[15] M. Christ, Weak type (1, 1) bounds for rough operators. Ann. of Math. 128 (1988), no. 1, 19-42. 2, 5, 6, 27 , 39

[16] M. Christ, $L^{p}$ bounds for spectral multipliers on nilpotent groups. Trans. Amer. Math. Soc. 328 (1991), no. $1,73-81.2$

[17] M. Christ and C.D. Sogge, The weak type $L^{1}$ convergence of eigenfunction expansions for pseudodifferential operators. Invent. Math. 94 (1988), no. 2, 421-453. 2, 5

[18] R. Coifman and G. Weiss, Analyse harmonique non-commutative sur certains espaces homogènes. Lecture Notes in Math. 242. Springer, Berlin-New York, 1971. 7

[19] T. Coulhon and A. Sikora, Gaussian heat kernel upper bounds via the Phragmén-Lindelöf theorem. Proc. Lond. Math. Soc. 96 (2008), no. 2, 507-544. 8, 20, 43

[20] M. Cowling, I. Doust, A. McIntosh and A. Yagi, Banach space operators with a bounded $H^{\infty}$ functional calculus. J. Austral. Math. Soc. Ser. A 60 (1996), no. 1, 51-89 20

[21] M. Cowling and A. Sikora, A spectral multiplier theorem for a sublaplacian on SU(2). Math. Z. 238 (2001), no. $1,1-36.2,5,12,13,16,17$

[22] E.B. Davies and B. Simon, $L^{p}$ norms of noncritical Schrödinger semigroups. J. Funct. Anal. 102 (1991), no. $1,95-115.43$

[23] X.T. Duong, E.M. Ouhabaz and A. Sikora, Plancherel-type estimates and sharp spectral multipliers. J. Funct. Anal. 196 (2002), no. 2, 443-485. 2, 5, 12, 13, 16, 41, 43, 46, 47

[24] X.T. Duong, A. Sikora and L.X. Yan, Weighted norm inequalities, Gaussian bounds and sharp spectral multipliers. J. Funct. Anal. 206 (2011), no. 4, 1106-1131.2

[25] C. Fefferman, Inequality for strongly singular convolution operators. Acta Math. 124 (1970), 9-36. 2, 3, 6

[26] C. Fefferman, The multiplier problem for the ball. Ann. of Math. 94 (1971), 330-336. 2

[27] C. Fefferman, A note on spherical summation multipliers. Israel of Math. 15 (1973), 44-52. 2, 3

[28] C. Guillarmou, A. Hassell and A. Sikora, Restriction and spectral multiplier theorems on asymptotically conic manifolds (2010), available at http://arXiv.org/abs/1012.3780. 2, 4, 10, 11, 13, 40

[29] A. Hassell and A. Sikora, Riesz transforms in one dimension. Indiana Univ. Math. J. 58 (2009), no. 2, 823-852. 43, 44

[30] W. Hebisch, Multiplier theorem on generalized Heisenberg groups. Colloq. Math. 65 (1993), no. 2, 231-239. 2,47

[31] L. Hörmander, The analysis of linear partial differential operators, I, II. Springer-Verlag, Berlin, 1983.

[32] L. Hörmander, On the Riesz means of spectral functions and eigenfunction expansions for elliptic differential operators. Some Recent Advances in the Basic Sciences, 2 (Proc. Annual Sci. Conf., Belfer Grad. School Sci., Yeshiva Univ., New Tork, 1965-1966), Belfer Graduate School Science, Yeshiva University, New York, $1969,155-202.2$

[33] L. Hörmander, Oscillatory integrals and multipliers on $F L^{p}$. Ark. Mat. 11 (1973), 1-11. 3

[34] L. Hörmander, The spectral function of an elliptic operator. Acta Math. 121 (1968), 193-218. 2

[35] L. Hörmander, Estimates for translation invariant operators in $L^{p}$ spaces. Acta Math. 104 (1960), 93-140. 2 
[36] G.B. Karadzhov, Riesz summability of multiple Hermite series in $L^{p}$ spaces. C. R. Acad. Bulgare Sci. 47 (1994), no. 2, 5-8. 42

[37] M. Keel and T. Tao, Endpoint Strichartz estimates. Amer. J. Math. 120 (1998), no. 5, 955-980. 7, 34, 37

[38] C. Kenig, R.J. Stanton and P. Tomas, Divergence of eigenfunction expansions. J. Funct. Anal. 46 (1982), no. $1,28-44.11$

[39] H. Koch and D. Tataru, $L^{p}$ eigenfunction bounds for the Hermite operator. Duke Math. J. 128 (2005), no. 2, $369-392.42$

[40] S. Lee, Improved bounds for Bochner-Riesz and maximal Bochner-Riesz operators. Duke Math. J. 122 (2004), no. 1, 205-232. 3

[41] V. Liskevich, Z. Sobol and H. Vogt, On the $L^{p}$ theory of $C^{0}$-semigroups associated with second-order elliptic operators II. J. Funct. Anal. 193 (2002), no. 1, 55-76. 41, 43

[42] W. Littman, C. McCarthy and N.M. Rivière, $L^{p}$-multiplier theorems. Studia Math. 30 (1968), 193-217. 4

[43] J. Marzuola, J. Metcalfe and D. Tataru, Strichartz estimates and local smoothing estimates for asymptotically flat Schrödinger equations. J. Funct. Anal. 255 (2008), no. 6, 1497-1553. 41

[44] G. Mauceri and S. Meda, Vector-valued multipliers on stratified groups. Rev. Mat. Iberoamericana 6 (1990), no. 3-4, 141-154. 2

[45] A. McIntosh, Operators which have an $H_{\infty}$ functional calculus, Miniconference on operator theory and partial differential equations (North Ryde, 1986), 210-231, Proceedings of the Centre for Mathematical Analysis, Australian National University, 14. Australian National University, Canberra, 1986. 20

[46] S.G. Mikhlin, Multidimensional singular integrals and integral equations, Pergamon Press, Oxford, 1965 (translated from the Russian by W. J. A. Whyte. Translation edited by I.N. Sneddon). 2

[47] D. Müller and E.M. Stein, On spectral multipliers for Heisenberg and related groups. J. Math. Pures Appl. 73 (1994), no. 4, 413-440.2, 47

[48] E.M. Ouhabaz, Analysis of heat equations on domains, London Math. Soc. Monographs, Vol. 31, Princeton Univ. Press (2005). 2

[49] I. Rodnianski, W. Schlag, Time decay for solutions of Schrödinger equations with rough and time-dependent potentials. Invent. Math. 155 (2004), no. 3, 451-513. 41

[50] A. Seeger, Endpoint inequalities for Bochner-Riesz multipliers in the plane. Pacific J. Math. 174 (1996), no. $2,543-553.2$

[51] A. Seeger, Endpoint estimates for multiplier transformations on compact manifolds. Indiana Univ. Math. J. 40 (1991), no. 2, 471-533. 6

[52] A. Seeger, Some inequalities for singular convolution operators in $L^{p}$-spaces. Trans. Amer. Math. Soc. 308 (1988), no. 1, 259-272. 4, 19

[53] A. Seeger and C.D. Sogge, On the boundedness of functions of (pseudo)-differential operators on compact manifolds. Duke Math. 59 (1989), no. 3, 709-736. 2, 4, 19

[54] A. Sikora, On the $L^{2} \rightarrow L^{\infty}$ norms of spectral multipliers of "quasi-homogeneous" operators on homogeneous groups. Trans. Amer. Math. Soc. 351 (1999), no. 9, 3743-3755. 2, 47

[55] A. Sikora, Riesz transform, Gaussian bounds and the method of wave equation. Math. Z. 247 (2004), no. 3, 643-662. 8, 20

[56] C.D. Sogge, On the convergence of Riesz means on compact manifolds. Ann. of Math. 126 (1987), no. 2, 439-447. 2, 3, 5, 17, 39

[57] C.D. Sogge, Fourier Integral in Classical Analysis. Cambridge University Press, Cambridge, 1993. 5, 17

[58] C.D. Sogge, Eigenfunction and Bochner Riesz estimates on manifolds with boundary. Math. Res. Lett. 9 (2002), no. 2, 205-216. 2, 5, 17, 39

[59] E. Stein, Interpolation of linear operators. Trans. Amer. Math. Soc. 83 (1956), 482-492. 2, 3

[60] E.M. Stein, Singular integrals and differentiability properties of functions. Princeton Mathematical Series, No. 30. Princeton Univ. Press, Princeton, NJ, 1970. 26

[61] E.M. Stein, Harmonic analysis: Real variable methods, orthogonality and oscillatory integrals. With the assistance of Timothy S. Murphy. Princeton Mathematical Series, 43. Monographs in Harmonic Analysis, III. Princeton University Press, Princeton, NJ, 1993 Princeton Univ. Press, Princeton, NJ, 1993. 2, 3

[62] T. Tao, Recent progress on the restriction conjecture (2003), available at http://arXiv.org/abs/0311181. 3

[63] T. Tao, Weak-type endpoint bounds for Riesz means. Proc. Amer. Math. Soc. 124 (1996), no. 9, 2797-2805. 2, 6, 27, 28, 39

[64] S. Thangavelu, Lecture on Hermite and Laguerre expansions. Princeton Univ. Press, Princeton, NJ, 1993 (with a preface by Robert S. Strichartz). 2 
[65] P. Tomas, A restriction theorem for the Fourier transform. Bull. Amer. Math. Soc. 81 (1975), 477-478. 3

[66] C. J. Tranter, Bessel functions with some physical applications. Hart Publishing Co. Inc., New York, (1969). 44

[67] M. Uhl, Spectral multiplier theorems of Hörmander type via generalized Gaussian estimates. PhD-Thesis, Karlsruher Institut für Technologie (2011). 7

[68] K. Yosida, Functional Analysis (Fifth edition). Spring-Verlag, Berlin, 1978. 11, 29

Peng Chen, Department of Mathematics, Sun Yat-sen (Zhongshan) University, Guangzhou, 510275, P.R. China

E-mail address: achenpeng1981@163.com

El Maati Ouhabaz, Institut de Mathématiques de Bordeaux, Université Bordeaux 1, UMR 5251, 351, Cours de la Libération 33405 Talence, France

E-mail address: Elmaati.Ouhabaz@math.u-bordeaux1.fr

Adam Sikora, Department of Mathematics, Macquarie University, NSW 2109, Australia

E-mail address: sikora@maths.mq.edu.au

Lixin Yan, Department of Mathematics, Sun Yat-sen (Zhongshan) University, Guangzhou, 510275, P.R. China

E-mail address: mcsylx@mail.sysu.edu.cn 